\title{
Higher-rank fields and currents
}

\author{
O.A. Gelfond ${ }^{a, b}$ and M.A. Vasiliev ${ }^{b}$ \\ ${ }^{a}$ Institute of System Research of Russian Academy of Sciences, \\ Nakhimovsky prospect 36-1, 117218, Moscow, Russia \\ ${ }^{b}$ I.E.Tamm Department of Theoretical Physics, Lebedev Physical Institute, \\ Leninsky prospect 53, 119991, Moscow, Russia \\ E-mail: gel@lpi.ru, vasiliev@lpi.ru
}

AbStRACT: $\operatorname{Sp}(2 M)$ invariant field equations in the space $\mathcal{M}_{M}$ with symmetric matrix coordinates are classified. Analogous results are obtained for Minkowski-like subspaces of $\mathcal{M}_{M}$ which include usual $4 d$ Minkowski space as a particular case. The constructed equations are associated with the tensor products of the Fock (singleton) representation of $\operatorname{Sp}(2 M)$ of any rank $\mathbf{r}$. The infinite set of higher-spin conserved currents multilinear in rank-one fields in $\mathcal{M}_{M}$ is found. The associated conserved charges are supported by $\left(\mathbf{r} M-\frac{\mathbf{r}(\mathbf{r}-1)}{2}\right)$-dimensional differential forms in $\mathcal{M}_{M}$, that are closed by virtue of the rank-2r field equations. The cohomology groups $H^{p}\left(\sigma_{-}^{\mathbf{r}}\right)$ with all $p$ and $\mathbf{r}$, which determine the form of appropriate gauge fields and their field equations, are found both for $\mathcal{M}_{M}$ and for its Minkowski-like subspace.

KEywords: Higher Spin Symmetry, Higher Spin Gravity, Scattering Amplitudes

ArXiv ePrint: 1312.6673 


\section{Contents}

1 Introduction 1

2 Young diagrams $\quad 5$

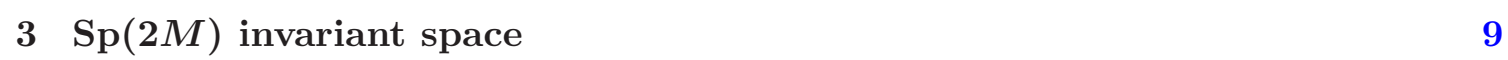

3.1 Fields and equations 9

$\begin{array}{llr}3.1 .1 & \text { Lower-rank examples } & 9\end{array}$

3.1.2 Any rank 11

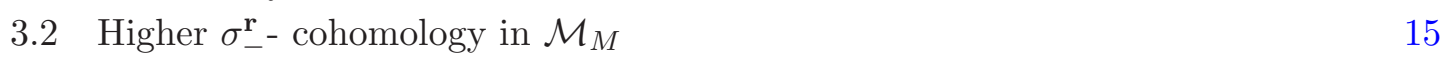

$\begin{array}{lll}3.2 .1 & \text { Main results } & 16\end{array}$

$\begin{array}{lll}3.3 & \text { Multilinear currents in } \mathcal{M}_{M} & 18\end{array}$

4 Minkowski-like reduction $\quad 21$

$\begin{array}{lll}4.1 & \text { Fields and equations in } \mathcal{M}_{M}^{M n k} & 21\end{array}$

$4.24 d$ Minkowski space 24

4.3 Higher $\sigma_{-}^{\mathbf{r}^{M n k}}$-cohomology in $\mathcal{M}_{M}^{M n k} \quad 25$

5 更--cohomology analysis $\quad 27$

$\begin{array}{ll}5.1 \text { Homotopy equation in } \mathcal{M}_{M} & 27\end{array}$

5.2 Sketch of the $\sigma_{-}$-cohomology analysis in $\mathcal{M}_{M}^{M n k} \quad 30$

6 Conclusion $\quad 32$

A Details of analysis of homotopy equation in $\mathcal{M}_{M} \quad 33$

\section{Introduction}

As originally observed by Fronsdal [1], infinite towers of massless fields in four dimensions admit an extension of the conformal algebra $\mathfrak{s u}(2,2)$ to $\mathfrak{s p}(4)$ allowing a description in the generalized matrix space $\mathcal{M}_{4}$ with symmetric matrix coordinates $X^{A B}=X^{B A}(A, B=$ $1, \ldots, 4)$. This observation was farther elaborated in [2-4]. The rank-one $\operatorname{Sp}(8)$-invariant unfolded equation describing massless fields of all spins is [4]

$$
\left(\xi^{A B} \frac{\partial}{\partial X^{A B}}+\sigma_{-}^{1}\right) \mathcal{C}(Y \mid X)=0, \quad \sigma_{-}^{1}=\xi^{A B} \frac{\partial^{2}}{\partial Y^{A} \partial Y^{B}},
$$

where $Y^{A}$ are auxiliary commuting variables that will be referred to as twistor variables. To simplify formulae we use notation $\xi^{A B}$ for anticommuting differentials $d X^{A B}$.

Note that eq. (1.1) admits an interesting interpretation in terms of world-like particle models of $[2,3]$, providing also a field-theoretical realization of the observation of 
Fronsdal [1] that the infinite tower of all $4 d$ massless fields enjoys $S p(8)$ symmetry. More generally $\mathcal{M}_{M}$ is the space with local coordinates $X^{A B}$ which are symmetric $M \times M$ matrices. $\mathrm{Sp}(2 M)$ invariant unfolded field equations corresponding to rank-r tensor products of the Fock (singleton) representation of $\operatorname{Sp}(2 M)$ were introduced in [5] where these equations were argued to describe "branes" of different dimensions in the $\operatorname{Sp}(2 M)$ invariant generalized space-time.

In [5], the case of rank-two equations

$$
\left(\xi^{A B} \frac{\partial}{\partial X^{A B}}+\sigma_{-}^{2}\right) \mathcal{C}(Y \mid X)=0
$$

where

$$
\sigma_{-}^{2}=\xi^{A B} \frac{\partial^{2}}{\partial Y_{1}^{A} \partial Y_{1}^{B}}+\xi^{A B} \frac{\partial^{2}}{\partial Y_{2}^{A} \partial Y_{2}^{B}}
$$

was considered in detail. In particular, all rank-two dynamical (primary) fields and field equations were found and it was shown that dynamical equations for most of the rank-two fields have the form of conservation conditions for conserved currents found in [6], which give rise to the full set of bilinear conserved charges in the rank-one theory.

Rank-r unfolded equations are

$$
\left(\xi^{A B} \frac{\partial}{\partial X^{A B}}+\sigma_{-}^{\mathbf{r}}\right) \mathcal{C}(Y \mid X)=0
$$

where

$$
\sigma_{-}^{\mathbf{r}}=\xi^{A B} \frac{\partial^{2}}{\partial Y_{k}^{A} \partial Y_{j}^{B}} \delta_{k j} \quad(k, j=1, \ldots, \mathbf{r}) .
$$

In this paper the analysis of [6] is extended to the fields and equations of arbitrary rank. Namely we find all dynamical fields, which are primary fields from the conformal field theory perspective, along with the explicit form of their field equations. It is shown that, similarly to the rank-two case, some of these fields give rise to differential forms that are closed by virtue of their field equations, thus generating conserved currents.

The $s p(2 M)$ invariant field equations are appropriate for description of infinite towers of massless fields that appear in higher-spin theories. For $M=2,4,8,16$, the $\operatorname{Sp}(2 M)$ invariant field equations describe towers of conformal massless fields in usual Minkowski spaces of dimensions $d=3,4,6$ and 10 , respectively $[3,7,8]$. Pattern of $\operatorname{Sp}(2 M)$ invariant field equations for other values $M$ so far has not been analysed including the case of $M=32$ which is most interesting in the context of $M$ theory.

The finite subsets of relativistic fields in $4 d$ Minkowski space are most conveniently described by the unfolded equations of motion for massless fields of all spins [4, 9],

$$
\xi^{\alpha \beta^{\prime}}\left(\frac{\partial}{\partial x^{\alpha \beta^{\prime}}}+i \frac{\partial^{2}}{\partial y^{\alpha} \partial \bar{y}^{\beta^{\prime}}}\right) \mathcal{C}(y, \bar{y} \mid x)=0 .
$$

Here $y^{\alpha}$ and $\bar{y}^{\beta^{\prime}}$ are auxiliary commuting complex conjugated two-component spinor variables $\left(\alpha, \beta=1,2 ; \alpha^{\prime}, \beta^{\prime}=1,2\right), x^{\alpha \beta^{\prime}}$ are Minkowski coordinates in two-component spinor 
notations, and $\xi^{\alpha \alpha^{\prime}}=d x^{\alpha \alpha^{\prime}}$ are anticommuting differentials. Equations (1.6) decompose into an infinite set of subsystems for fields of different helicities $h$

$$
\mathcal{C}\left(\mu y, \mu^{-1} \bar{y} \mid x\right)=\mu^{2 h} \mathcal{C}(y, \bar{y} \mid x) .
$$

The space with coordinates $x^{\alpha \beta^{\prime}}$ with $\alpha=1, \ldots, K$ and $\alpha^{\prime}=1, \ldots, K$ for any $K$ we call generalized Minkowski space $\mathcal{M}_{2 K}^{M n k}$. The rank-r generalization of (1.6) is

$$
\xi^{\alpha \beta^{\prime}}\left(\frac{\partial}{\partial x^{\alpha \beta^{\prime}}}+i \frac{\partial^{2}}{\partial y_{k}^{\alpha} \partial \bar{y}_{j}^{\beta^{\prime}}} \eta^{k j}\right) \mathcal{C}(y, \bar{y} \mid x)=0
$$

for any Hermitian form $\eta^{k j}, k, j=1, \ldots, \mathbf{r}, \alpha, \alpha^{\prime}=1, \ldots, K$. Though interpretation of these equations for higher $K$ from the perspective of usual Minkowski space embedded into $\mathcal{M}_{2 K}^{M n k}$ demands more detailed analysis which is beyond the scope of this paper, we briefly comment on the cases of $K=2,4$ and 8 being reductions of the $\operatorname{Sp}(2 M)$ systems with $M=4,8$ and 16 , respectively. ${ }^{1}$

The case of $K=2$ gives the genuine $4 d$ Minkowski space.

The case of $K=4$ results from the reduction of the $\operatorname{Sp}(16)$ invariant system. The latter was shown in $[7,8]$ to describe conformal massless fields in the $6 d$ Minkowski space carrying spinning degrees of freedom valued in $\mathrm{SU}(2)$. As emphasized in [8] this implies that the original $\mathrm{Sp}(16)$ invariant system describes an infinite tower of $6 d$ massless fields of all spins such that the multiplicity of a spin $s$ is $2 s+1$ coinciding with the dimension of the spin- $s$ representation of the spinning $\mathrm{SU}(2)$. Coordinates $x^{\alpha \beta^{\prime}}$ can be interpreted as the part of the coordinates $X^{A B}$ that are invariant under the action of one of the generators $\mathcal{H}$ in the spinning $\mathfrak{s u}(2)$, that acts on the primed and unprimed indices by the conjugated phase transformations. As a result, the irreducible subsystems in $\mathcal{M}_{8}^{M n k}$ are characterized by $\mathfrak{s l}(2)$ helicities $h$. Each of these systems contains an infinite tower of conformal fields in which every spin $s \geq|h|$ appears once ( $s$ is related to the length of the rectangular Young diagram associated with a $6 d$ conformal field in Minkowski space).

The case of $K=8$ results from the reduction of the $\mathrm{Sp}(32)$ invariant system. The latter describes a tower of conformal massless self-dual fields of all spins in the $10 d$ Minkowski space [8] such that each spin appears once. Now the reduction of the $\operatorname{Sp}(32)$ invariant system to $\mathcal{M}_{2 K}^{M n k}$ has different interpretation. Since indices of the coordinates $X^{A B}$ of $\mathcal{M}_{16}$ are associated with $10 d$ chiral spinors, the coordinates $x^{\alpha \beta^{\prime}}$ cannot be obtained by a $10 d$ Lorentz covariant projection from $X^{A B}$. In other words, the helicity-like operator distinguishing between primed and unprimed indices cannot be $10 d$ Lorentz covariant. Hence, the $\mathcal{M}_{16}^{M n k}$ setup must break manifest $10 d$ Lorentz covariance describing the system in a Minkowski space of some lower dimension $d<10$. The simplest option is to identify the helicity-like generator with one of the $10 d$ rotations, considering the system in eight dimensions. An interesting alternative possibility to be explored is to embed a $9 d$ Minkowski space in $\mathcal{M}_{16}^{M n k}$ using another helicity operator that would partially act in the spinning space. In both of these cases the $10 d$ conformal invariance will be a kind of hidden and the

\footnotetext{
${ }^{1}$ We are grateful to the referee for raising this question.
} 
same time the self-dual $10 d$ fields will be traded for lower-dimensional fields not restricted by the self-duality conditions. We hope to elaborate details of this analysis elsewhere.

In this paper we analyse the pattern of Minkowski-like equations with general $\mathbf{r}$ and $K$ from the perspective of the generalized space $\mathcal{M}_{2 K}^{M n k}$ with no reference to the underlying physical space. However, since in the particular case of $K=2$ the space $\mathcal{M}_{4}^{M n k}$ is the usual $4 d$ Minkowsky space, this allows us to derive all conformal primary currents in the four-dimensional Minkowski space that are built from $4 d$ massless fields of all spins. (These results have been already announced and used in [10] for the analysis of the operator algebra and correlators of conserved currents in four dimensions.)

We expect that results of the present paper may have applications in the context of $A d S / C F T$ holography [11-13] and especially, higher-spin holography (see, e.g., [14-25] and references therein) because, as emphasized in [26], the duality between fields in higher dimensions and currents in lower dimensions to large extent amounts in the language of this paper to the duality between lower-rank fields in $\mathcal{M}_{M}$ with higher $M$ and higher-rank fields in $\mathcal{M}_{M}$ with lower $M$.

Another interesting application is to the analysis of multiparticle amplitudes (see e.g. [27] and references therein). The key fact here is that the product of $r$ rank-one solutions

$$
\mathcal{C}\left(y_{i}, \bar{y}_{i} \mid x\right)=\mathcal{C}\left(y_{1}, \bar{y}_{1} \mid x\right) \mathcal{C}\left(y_{2}, \bar{y}_{2} \mid x\right) \ldots \mathcal{C}\left(y_{\mathbf{r}}, \bar{y}_{\mathbf{r}} \mid x\right)
$$

gives a solution to the rank-r equation. In these terms an $\mathbf{r}$-particle amplitude represents a solution to the rank-r system. From this perspective the constructed conserved multiparticle charges may be of most interest since amplitudes supported by such charges can be represented as multiple integrals independent of local variations of the integration cycle. In this language the nontrivial dynamics of a model in question should be hidden in the so-called parameters which in our formalism are arbitrary functions of certain twistor variables, which parameterize different amplitudes. We hope to come back to a more detailed analysis of this issue in a future publication.

The analysis of the pattern of dynamical fields and their field equations is performed in the $\sigma_{-}$-cohomology language which is analogous (and in many cases equivalent) to the search of singular vectors of conformal modules. Specifically, zero-form fields and their field equations are classified by $H^{0}\left(\sigma_{-}\right)$and $H^{1}\left(\sigma_{-}\right)$, respectively. In this paper, we find all cohomology groups $H^{p}\left(\sigma_{-}\right)$with $p \geq 0$. These results determine the form of appropriate gauge fields and their field equations both for $\mathcal{M}_{M}$ and for its Minkowski-like subspace $\mathcal{M}_{M}^{M n k}$.

The rest of the paper is organized as follows. Section 2 contains our Young diagram conventions and some their properties. In particular, the structure of differential forms in $\mathcal{M}_{M}$ is analyzed here. Section 3 presents the full list of dynamical fields and field equations of any rank-r in $\mathcal{M}_{M}$. Details of derivation of the equations of motion as well as the form of multilinear conserved currents in $\mathcal{M}_{M}$ are also given here. Section 4 contains summary of our results for conformal fields and their field equations both in the usual four-dimensional Minkowski space and in the generalized Minkowski space $\mathcal{M}_{M}^{M n k}$. Section 5 contains the

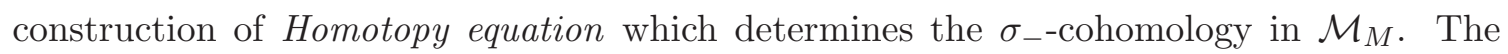


main tool in the analysis of Homotopy equation is the South-West principle allowing to minimize the positive semi-definite homotopy operators. Details of the analysis of the $\sigma_{-}-$ cohomology in $\mathcal{M}_{M}^{M n k}$ are sketched in section 5.2. In section 6 some perspectives are briefly discussed. Appendix A contains details of the analysis of the $\sigma_{-}$-cohomology in $\mathcal{M}_{M}$.

\section{Young diagrams}

A tensor $A_{B_{1}^{1} \ldots B_{l_{1}}^{1}, \ldots, B_{1}^{m} \ldots B_{l_{m}}^{m}}\left(l_{1} \geq \ldots \geq l_{m}\right)$ obeys symmetry properties of the Young diagram (YD) $\mathbf{Y}\left(l_{1}, \ldots, l_{m}\right)$ with manifest symmetrization provided that $A$ is symmetric with respect to permutations of $l_{k}$ indices $B_{1}^{k}, \ldots, B_{l_{k}}^{k}$ of any $k$-th row, while the symmetrization over $l_{k}+1$ indices $B_{1}^{k}, \ldots, B_{l_{k}}^{k}, B_{j}^{p}$ yields zero for any $p>k$. Such tensors are conventionally denoted $A_{B^{1}\left(l_{1}\right), \ldots, B^{m}\left(l_{m}\right)}$.

Analogously, a tensor $A_{B_{1}^{1}, \ldots, B_{1}^{h_{1}} ; \ldots ; B_{p}^{1}, \ldots, B_{p}^{h_{p}}}\left(h_{1} \geq \ldots \geq h_{p}\right)$ obeys the symmetry properties of the YD $\mathbf{Y}\left[h_{1}, \ldots, h_{p}\right]$ with manifest antisymmetrization provided that it is antisymmetric with respect to permutations of $h_{k}$ indices $B_{k}^{1}, \ldots, B_{k}^{h_{k}}$ of any $k$-th column while the antisymmetrization over $h_{k}+1$ indices $B_{k}^{1}, \ldots, B_{k}^{h_{k}}, B_{q}^{j}$ yields zero for any $q>k$. Such tensors are conventionally denoted $A_{B_{1}\left[h_{1}\right], \ldots, B_{p}\left[h_{p}\right]}$.

A height of the $k$-th column of $\mathbf{Y}$ is denoted $\mathrm{h}_{k}(\mathbf{Y})$ or simply $h_{k}$, while a length of the $k$-th row of $\mathbf{Y}$ is denoted $l_{k}(\mathbf{Y})$ or $l_{k}$. Let us stress that components of a tensor with symmetry properties of any Young diagram with manifest symmetrization are linear combinations of the components of a tensor with symmetry properties of the same Young diagram with manifest antisymmetrization and vise versa. Weight $|\mathbf{Y}|$ equals to the number of cells of a YD $\mathbf{Y}\left[h_{1}, \ldots, h_{p}\right]$, i.e.,

$$
|\mathbf{Y}|:=h_{1}+\ldots+h_{p}=l_{1}+\ldots+l_{m} .
$$

Any Young diagram can be represented as the unification of its elementary cells $\mathcal{S}(i, j)$ on the intersection of its $i-t h$ row and $j-t h$ column, i.e., $\mathbf{Y}=\bigcup_{\mathcal{S}(i, j) \in \mathbf{Y}} \mathcal{S}(i, j)$. For any cell $\mathcal{S}(i, j)$ and parameter $a \in \mathbb{R}$ we introduce the characteristic function

$$
\chi^{a}(\mathcal{S}(i, j))=(j-i+a) .
$$

For any set of cells $\mathcal{A}$ we introduce its characteristic function ${ }^{2}$

$$
\chi^{a}(\mathcal{A}):=\sum_{\mathcal{S}(i, j) \in \mathcal{A}} \chi^{a}(\mathcal{S}(i, j))=\sum_{\mathcal{S}(i, j) \in \mathcal{A}}(j-i+a) .
$$

Considering a diagram $\mathbf{Y}\left[h_{1}, \ldots, h_{k}\right]$ as the unification of either its columns $\mathbf{H}_{j}\left(h_{j}\right)$ or rows $\mathbf{L}_{j}\left(l_{j}\right)$ we obtain

$$
\chi^{a}(\mathbf{Y})=\sum_{\mathbf{H}_{j} \subseteq \mathbf{Y}} \sum_{\mathcal{S}(i, j) \in \mathbf{H}_{j}}(j-i+a)=-\frac{1}{2} \sum_{j} h_{j}\left(h_{j}-2 j+1-2 a\right)
$$

\footnotetext{
${ }^{2}$ We are grateful to Andrey Mironov for bringing to our attention the paper [28] where the function $n_{\lambda}=2 \chi^{0}(\lambda)$ characterized YD $\lambda$ was used.
} 
and

$$
\chi^{a}(\mathbf{Y})=\sum_{\mathbf{L}_{i} \subseteq \mathbf{Y}} \sum_{\mathcal{S}(i, j) \in \mathbf{L}_{i}}(j-i+a)=\frac{1}{2} \sum_{i} l_{i}\left(l_{i}-2 i+1+2 a\right) .
$$

Hence, the right-hand sides of (2.4) and (2.5) are equal for any YD Y.

Cells of a Young diagram can be ordered as follows: for any two cells $\mathcal{S}_{1}$ and $\mathcal{S}_{2}$, $\mathcal{S}_{1} \prec \mathcal{S}_{2}$ if $\chi^{a}\left(\mathcal{S}_{1}\right)<\chi^{a}\left(\mathcal{S}_{2}\right)$ and $\mathcal{S}_{1} \preceq \mathcal{S}_{2}$ if $\chi^{a}\left(\mathcal{S}_{1}\right) \leq \chi^{a}\left(\mathcal{S}_{2}\right)$. (Note that this definition is insensitive to $a$.) Definition (2.3) implies that, with respect to this ordering, for any two cells the smaller is situated South-West to the larger.

If $\mathcal{S}_{1}^{i} \prec \mathcal{S}_{2}^{i}\left(\mathcal{S}_{1}^{i} \preceq \mathcal{S}_{2}^{i}\right)$ for $i=1, \ldots, N$ then $\mathcal{A}_{1} \prec \mathcal{A}_{2}\left(\mathcal{A}_{1} \preceq \mathcal{A}_{2}\right)$ where $\mathcal{A}_{1,2}=$ $\bigcup_{i \leq N} \mathcal{S}_{1,2}^{i}$. Thus the partial ordering can be introduced in particular for Young diagrams containing equal numbers of cells.

The symmetrized tensor product of a number of rank-two symmetric tensors $\delta_{i j}$ described by the YD Y $[1,1]$ decomposes into a linear combination of tensors with the symmetry properties

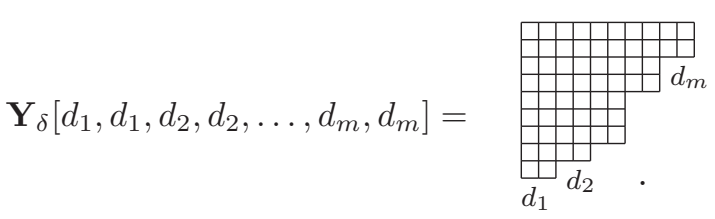

This is because antisymmetrization of indices $i_{1}, i_{2}, \ldots, i_{d}$, say, in $\delta_{i_{1} j_{1}} \ldots \delta_{i_{d} j_{d}}$ implies antisymmetrization of indices $j_{1}, j_{2}, \ldots, j_{d}$. Components of the symmetrized tensor product of a number of $\mathbf{Y}[1,1]$

$$
\mathbf{Y}_{\delta}\left[d_{1}, d_{1}, \ldots, d_{m}, d_{m}\right] \in\left(\otimes_{\text {sym }} \mathbf{Y}[1,1]\right)
$$

will be referred to as Kronecker diagrams.

On the other hand, as shown below, the antisymmetrized tensor product of tensors with the symmetry properties of $\mathbf{Y}[1,1]$ contains various tensors with the symmetry properties of almost symmetric Young diagrams $\mathbf{Y}_{A}$ defined as follows. For any Young diagram invariant under reflection with respect to the diagonal, one cell should be added to each row that intersects the diagonal. Pictorially, shading the added cells,

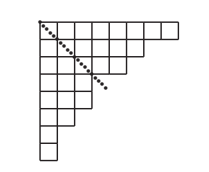

Symmetric $\mathbf{Y}_{S}$

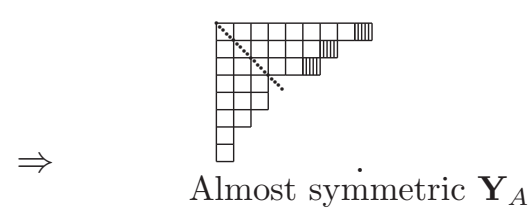

Almost symmetric $\mathbf{Y}_{A}$

The simplest one is the almost symmetric hook of height $h$

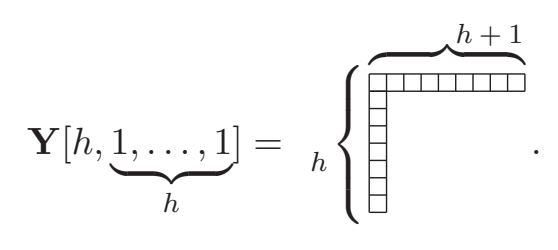


For instance, the full list of almost symmetric Young diagrams belonging to $\otimes_{\text {asym }}^{n} \mathbf{Y}[1,1]$ with $n \leq 5$ is

- $\mathrm{n}=1: \quad \mathbf{Y}(2) \square \quad ;$

- $\mathrm{n}=2: \quad \mathbf{Y}(3,1) \square^{\square}$;

- $\mathrm{n}=3: \quad \mathbf{Y}(4,1,1)=\Theta^{\square}$ and $\mathbf{Y}(3,3)=\boxplus ;$

- $\mathrm{n}=4: \quad \mathbf{Y}(5,1,1,1)=\rrbracket$ and $\mathbf{Y}(4,3,1)=\boxplus$;

- $\mathrm{n}=5: \quad \mathbf{Y}(6,1,1,1,1)=\bigoplus \quad, \mathbf{Y}(5,3,1,1)=\bigoplus$ and $\mathbf{Y}(4,4,2)=\bigoplus$.

Any almost symmetric diagram $\mathbf{Y}_{A}(2.8)$ admits a nested hook realization, namely $\mathbf{Y}_{A}$ is a unification of a set of shifted almost symmetric hooks $\mathcal{Y}\left(k, a_{k}\right)$

$$
\mathbf{Y}_{A}=\mathbb{Y}^{\text {nest }}\left\{a_{1}, \ldots a_{\mathbf{n}}\right\}=\bigcup_{1 \leq k \leq \mathbf{n}} \mathcal{Y}\left(k, a_{k}\right), \quad a_{j} \geq a_{j+1}+1, \quad a_{\mathbf{n}} \geq 1
$$

where $\mathcal{Y}\left(k, a_{k}\right)$ is the almost symmetric hook shifted by $k-1$ cells down along the diagonal. More precisely,

$$
\mathcal{Y}\left(k, a_{k}\right)=\bigcup_{j=1}^{a_{k}}(\mathcal{S}(k, k+j) \bigcup \mathcal{S}(j+k-1, k)) .
$$

For example, $\mathcal{Y}(1, h)=\mathbf{Y}[h, \underbrace{1, \ldots, 1}_{h}](2.9)$.

Pictorially, in the almost symmetric diagram shown below the bolded almost symmetric hook is $\mathcal{Y}(2,5)$ while the other two are $\mathcal{Y}(1,8)$ and $\mathcal{Y}(3,3)$

$$
\mathbb{Y}^{\text {nest }}\{8,5,3\}=\mathcal{Y}(1,8) \cup \mathcal{Y}(2,5) \cup \mathcal{Y}(3,3)=
$$

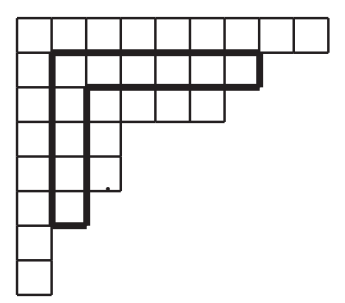

Note that by definition

$$
\mathrm{h}_{\mathbf{n}+1}\left(\mathbb{Y}^{\text {nest }}\left\{a_{1}, \ldots a_{\mathbf{n}}\right\}\right)=\mathbf{n}:=\sharp\left(\mathbb{Y}^{\text {nest }}\left\{a_{1}, \ldots a_{\mathbf{n}}\right\}\right) .
$$

For instance, the height of 4-th column of $\mathbb{Y}^{\text {nest }}\{8,5,3\}(2.12)$ is 3 .

The nested realization (2.10) of almost symmetric diagrams $\mathbf{Y}_{A}(2.8)$ yields that all of them obey

$$
\chi^{q}\left(\mathbf{Y}_{A}\right)=\left(q+\frac{1}{2}\right)\left|\mathbf{Y}_{A}\right|
$$


Indeed, by definition (2.2) any pair of cells symmetric with respect to the diagonal does not contribute to $\chi^{0}$, hence for an almost symmetric shifted hook $\mathcal{Y}\left(k, h_{k}\right)(2.11)$

$$
\chi^{0}\left(\mathcal{Y}\left(k, h_{k}\right)\right)=h_{k}=\frac{1}{2}\left|\mathcal{Y}\left(k, h_{k}\right)\right| .
$$

Summation over all shifted almost symmetric hooks gives (2.14).

It is convenient to introduce block hooks of the form $\mathbb{Y}^{\text {nest }}\{\underbrace{m, m-1, \ldots, m-n}_{n+1}\}$ with arbitrary integer $m>n$. Pictorially,
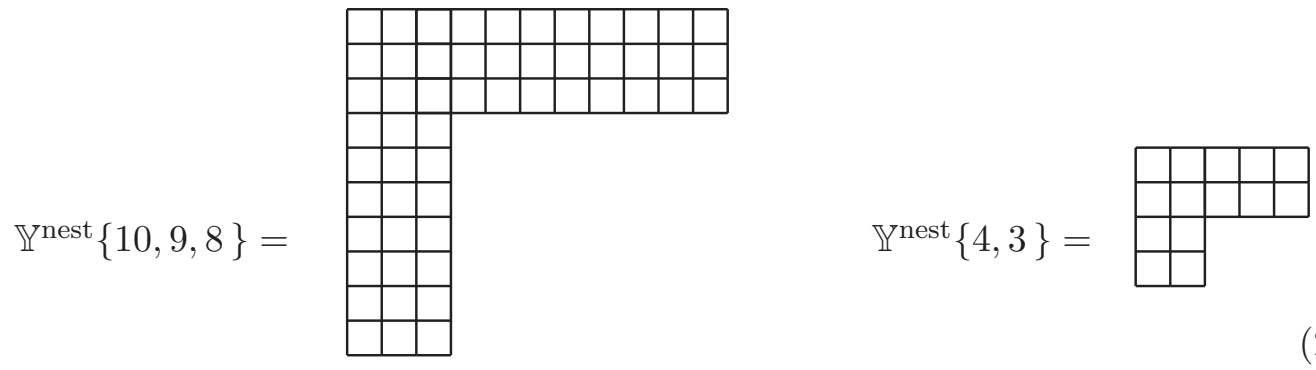

A general almost symmetric diagram can be treated as nested block hooks consisting of $\mathbf{p}$ block hooks

$$
\mathbf{Y}_{A}=\mathbb{Y}^{\text {nest }}\{\underbrace{\mathbf{a}_{1}, \mathbf{a}_{1}-1, \ldots, \mathbf{a}_{1}-n_{1}+1}_{n_{1}}, \ldots, \underbrace{\mathbf{a}_{\mathbf{p}}, \mathbf{a}_{\mathbf{p}}-1, \ldots, \mathbf{a}_{\mathbf{p}}-n_{\mathbf{p}}+1}_{n_{\mathbf{p}}}\}
$$

under conditions

$$
n_{1}+\ldots+n_{\mathbf{p}}=\mathbf{n}, \quad \mathbf{a}_{i}-n_{i}>\mathbf{a}_{i+1}>0 \quad \text { for } i+1 \leq \mathbf{p}, \quad \mathbf{a}_{\mathbf{p}} \geq n_{\mathbf{p}},
$$

where $n_{i}$ is the number of hooks in the $i^{\text {th }}$ block and $\mathbf{n}$ is the total number of hooks in $\mathbf{Y}_{A}$.

Let $\xi^{A B}$ be anticommuting differentials

$$
\xi^{A B}=\xi^{B A}, \quad \xi^{A B} \xi^{C D}=-\xi^{C D} \xi^{A B} .
$$

The coefficients of differential forms

$$
Q_{A_{1} B_{1} ; \ldots ; A_{n} B_{n}} \xi^{A_{1} B_{1}} \ldots \xi^{A_{n} B_{n}}
$$

belong to the space of antisymmetric tensor products of $Y[1,1]$. To show that they are described by almost symmetric diagrams we observe that anticommutativity of the differentials implies that the projection of $\xi^{A B} \xi^{C D}$ to the window diagram is zero

$$
\left.\xi^{A B} \xi^{C D}\right|_{\boxplus}=0
$$

From here it follows that the product of $n$ variables $\xi$ antisymmetrized over $n$ indices has the symmetry of the hook $\mathbf{Y}[n, \underbrace{1, \ldots, 1}_{n}]$. Indeed, antisymmetrization over $n$ indices of 
$n$ variables $\xi$ implies symmetrization over the other $n$ indices. There are two diagrams containing a symmetrization over $n$ indices

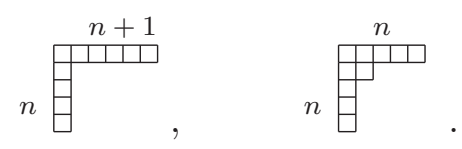

However the one containing window is zero by (2.19). Due to antisymmetrization in every column, this implies that any Young diagram associated with a differential form has the nested hook structure, i.e., is almost symmetric.

\section{$3 \quad \operatorname{Sp}(2 M)$ invariant space}

\subsection{Fields and equations}

\subsubsection{Lower-rank examples}

From the rank-one $\operatorname{Sp}(2 M)$-invariant unfolded equation (1.1) it follows that most of the component fields in the expansion

$$
\mathcal{C}(Y \mid X)=\sum_{n=0}^{\infty} Y^{A_{1}} \ldots Y^{A_{n}} C_{A_{1} \ldots A_{n}}(X)
$$

are reconstructed in terms of ( $X$-derivatives of $)$ the primary fields that satisfy

$$
\sigma_{-}^{1} C(Y \mid X)=0 .
$$

In the rank-one case, the primary fields are [4]

$$
C(X):=\mathcal{C}(0 \mid X), \quad C_{A}(X) Y^{A} .
$$

The symmetry properties of $C$ and $C_{A}$ are represented by the Young diagrams $\bullet$ (empty diagram) and $\square$, respectively.

The following equations hold as a consequence of unfolded equations (1.1):

$$
\begin{gathered}
\left(\frac{\partial}{\partial X^{A C}} \frac{\partial}{\partial X^{B D}}-\frac{\partial}{\partial X^{B C}} \frac{\partial}{\partial X^{A D}}\right) \mathcal{C}(Y \mid X)=0 \\
\left(\frac{\partial}{\partial X^{B D}} \frac{\partial}{\partial Y^{A}}-\frac{\partial}{\partial X^{A D}} \frac{\partial}{\partial Y^{B}}\right) \mathcal{C}(Y \mid X)=0
\end{gathered}
$$

and, in particular,

$$
\begin{aligned}
\left(\frac{\partial}{\partial X^{A C}} \frac{\partial}{\partial X^{B D}}-\frac{\partial}{\partial X^{B C}} \frac{\partial}{\partial X^{A D}}\right) C(X) & =0, \\
\frac{\partial}{\partial X^{B D}} C_{A}(X)-\frac{\partial}{\partial X^{A D}} C_{B}(X) & =0 .
\end{aligned}
$$

The symmetry properties of the left-hand sides of equations (3.5) and (3.6) are represented by the Young diagrams $\boxplus$ and $\boxplus$, respectively. In the language of $\sigma_{-}$-cohomology [29] (see also [30]) convenient for the analysis of the pattern of zero-form higher-rank fields, 
primary fields and their field equations are represented by the cohomology groups $H^{0}\left(\sigma_{-}^{1}\right)$ and $H^{1}\left(\sigma_{-}^{1}\right)$, respectively. Hence, the structure of rank-one fields and field equations is represented by the following diagrams

\begin{tabular}{|c|c|c|}
\hline$H^{0}\left(\sigma_{-}^{1}\right): \mathbf{Y}_{0}$ & $\bullet$ & $\square$ \\
\hline$H^{1}\left(\sigma_{-}^{1}\right): \mathbf{Y}_{1}$ & $\boxplus$ & $\Xi$ \\
\hline
\end{tabular}

Note that eqs. (3.5), (3.6) are the only independent equations obeyed by the primary (=dynamical) fields as a consequence of (1.1). Such equations will be referred to as dynamical.

Rank-two unfolded equations (1.2) are most conveniently analyzed in terms of variables

$$
2 z^{A}=Y_{1}^{A}+i Y_{2}^{A}, \quad 2 \bar{z}^{A}=Y_{1}^{A}-i Y_{2}^{A}
$$

with $\sigma_{-}^{2}(1.2)$ taking the form

$$
\sigma_{-}^{2}=\xi^{A B} \frac{\partial^{2}}{\partial z^{A} \partial \bar{z}^{B}} .
$$

The following homogeneous differential equations hold as a consequence of (3.9)

$$
\begin{aligned}
& \varepsilon^{B_{1}, B_{2}, B_{3}} \frac{\partial^{3}}{\partial X^{B_{1} D_{1}} \partial X^{B_{2} D_{2}} \partial X^{B_{3} D_{3}}} \mathcal{C}(z, \bar{z} \mid X)=0, \\
& \varepsilon^{B_{1}, B_{2}, B_{3}} \frac{\partial^{3}}{\partial X^{B_{1} D} \partial z^{B_{2}} \partial \bar{z}^{B_{3}}} \mathcal{C}(z, \bar{z} \mid X)=0, \\
& \varepsilon^{B_{1}, B_{2}, B_{3}} \frac{\partial^{3}}{\partial X^{B_{1} D_{1}} \partial X^{B_{2} D_{2}} \partial z^{B_{3}}} \mathcal{C}(z, \bar{z} \mid X)=0, \\
& \varepsilon^{B_{1}, B_{2}, B_{3}} \frac{\partial^{3}}{\partial X^{B_{1} D_{1}} \partial X^{B_{2} D_{2}} \partial \bar{z}^{B_{3}}} \mathcal{C}(z, \bar{z} \mid X)=0, \\
& \varepsilon^{A, B} \varepsilon^{C, D} \frac{\partial^{3}}{\partial X^{B C} \partial z^{A} \partial z^{D}} \mathcal{C}(z, \bar{z} \mid X)=0, \\
& \varepsilon^{A, B} \varepsilon^{C, D} \frac{\partial^{3}}{\partial X^{B C} \partial \bar{z}^{A} \partial \bar{z}^{D}} \mathcal{C}(z, \bar{z} \mid X)=0,
\end{aligned}
$$

where arbitrary rank- $m$ totally antisymmetric tensors $\varepsilon^{A_{1}, \ldots, A_{m}}$ are introduced to impose appropriate antisymmetrizations.

As shown in [5], the rank-two primary fields are

$$
C_{A_{1} \ldots A_{m}}(X) z^{A_{1}} \ldots z^{A_{m}}, \quad \bar{C}_{A_{1} \ldots A_{m}}(X) \bar{z}^{A_{1}} \ldots \bar{z}^{A_{m}}, \quad C_{A, B}(X) z^{A^{B}} \bar{z}^{B} \quad\left(C_{A, B}=-C_{B, A}\right) .
$$

Dynamical equations for the primary fields are [5]

$$
\begin{array}{rlrl}
\varepsilon^{B_{1}, B_{2}, B_{3}} \frac{\partial}{\partial X^{B_{1} D_{1}}} \frac{\partial}{\partial X^{B_{2} D_{2}}} \frac{\partial}{\partial X^{B_{3} D_{3}}} C(X) & =0, & \varepsilon^{B_{1}, B_{2}, B_{3}} \frac{\partial}{\partial X^{B_{1} D}} C_{B_{2}, B_{3}}(X)=0 \\
\varepsilon^{B_{1}, B_{2}, B_{3}} \frac{\partial}{\partial X^{B_{1} D_{1}}} \frac{\partial}{\partial X^{B_{2} D_{2}}} C_{B_{3}}(X)=0, & \varepsilon^{B_{1}, B_{2}, B_{3}} \frac{\partial}{\partial X^{B_{1} D_{1}}} \frac{\partial}{\partial X^{B_{2} D_{2}}} \bar{C}_{B_{3}}(X)=0 \\
\varepsilon^{E, B} \varepsilon^{C, D} \frac{\partial}{\partial X^{B C}} \bar{C}_{E D A(m-2)}(X)=0, & \varepsilon^{E, B} \varepsilon^{C, D} \frac{\partial}{\partial X^{B C}} C_{E D A(m-2)}(X)=0 .
\end{array}
$$


Hence, the list of the Young diagrams associated with $H^{0}\left(\sigma_{-}^{2}\right)$ and $H^{1}\left(\sigma_{-}^{2}\right)$ is

\begin{tabular}{|c|c|c|c|c|}
\hline$H^{0}\left(\sigma_{-}^{2}\right): \mathbf{Y}_{0}$ & $\bullet$ & $\square$ & $\boxminus$ & $2+n$ \\
\hline$H^{1}\left(\sigma_{-}^{2}\right): \mathbf{Y}_{1}$ & $\boxplus$ & $\bigoplus$ & $\bigoplus$ & 巴I \\
\hline
\end{tabular}

Fields (3.11) and equations (3.12) are in one-to-one correspondence with elements of $H^{0}\left(\sigma_{-}^{2}\right)$ and $H^{1}\left(\sigma_{-}^{2}\right)$, respectively. The latter are tensorial spaces with respect to indices $A, B \ldots=1, \ldots M$, characterized by the Young diagrams of (3.13).

One observes that in the examples of ranks one and two, dynamical fields associated with $H^{0}\left(\sigma_{-}^{\mathbf{r}}\right)$ are such that the total number of indices in the first two columns of the respective Young diagrams does not exceed $\mathbf{r}$.

Another property illustrated by these examples is that all columns starting from the third one of the Young diagrams $\mathbf{Y}_{0}$ and $\mathbf{Y}_{1}$ associated with $H^{0}\left(\sigma_{-}^{\mathbf{r}}\right)$ and $H^{1}\left(\sigma_{-}^{\mathbf{r}}\right)$ are equal, while the first two columns are such that together they form a rectangular two-column block of height $\mathbf{r}+1$, i.e.,

$$
\mathrm{h}_{1}\left(\mathbf{Y}_{0}\right)+\mathrm{h}_{2}\left(\mathbf{Y}_{1}\right)=\mathrm{h}_{2}\left(\mathbf{Y}_{0}\right)+\mathrm{h}_{1}\left(\mathbf{Y}_{1}\right)=\mathbf{r}+1, \quad \mathrm{~h}_{k}\left(\mathbf{Y}_{0}\right)=\mathrm{h}_{k}\left(\mathbf{Y}_{1}\right) \quad \forall k \geq 3,
$$

where $\mathrm{h}_{k}\left(\mathbf{Y}_{i}\right)$ is the height of the $k^{\text {th }}$ column of $\mathbf{Y}_{i}$.

We say that a pair of Young diagrams are rank-r two-column dual if they obey (3.14). The rank-one and two examples suggest that dynamical fields and their field equations are described by rank-r two-column dual Young diagrams. As we explain now this is indeed true for fields of any rank.

\subsubsection{Any rank}

Rank-r unfolded equations (1.4) are $\mathfrak{s p}(2 M)$ symmetric by the general argument of [5]. Indeed, it is well known (for more details see e.g. [4]) that any system of equations of the form

$$
(\mathrm{d}+\omega) C(X)=0, \quad \mathrm{~d}:=d X^{\mathcal{A}} \frac{\partial}{\partial X^{\mathcal{A}}},
$$

where $C(X)$ is some set of $p$-forms taking values in a $\mathfrak{g}$-module $\mathcal{V}$ and the one-form $\omega(X)=$ $d X^{\mathcal{A}} \omega_{\mathcal{A}}(X)$ is some fixed connection of $\mathfrak{g}$ obeying the flatness condition

$$
\mathrm{d} \omega+\frac{1}{2}[\omega, \wedge \omega]=0
$$

([, ] denotes the Lie product in $\mathfrak{g})$, is invariant under the global symmetry $\mathfrak{g}$.

Eq. (1.4) is a particular case of eq. (3.15) with $\mathfrak{g}=\mathfrak{s p}(2 M)$. The generators of $\mathfrak{s p}(2 M)$ can be realized as bilinears built from oscillators $Y_{i}^{A}$ and $W_{A}^{i}=\frac{\partial}{\partial Y_{i}^{A}}$,

$$
\begin{aligned}
{\left[W_{A}^{i}, Y_{j}^{B}\right] } & =\delta_{j}^{i} \delta_{A}^{B}, \quad\left[Y_{i}^{A}, Y_{j}^{B}\right]=0, \quad\left[W_{A}^{i}, W_{B}^{i}\right]=0, \quad i, j=1 \ldots \mathbf{r}, \\
T^{A B} & =Y_{i}^{A} Y_{j}^{B} \delta^{i j}, \quad T_{A B}=W_{A}^{i} W_{B}^{j} \delta_{i j}, \quad T_{B}^{A}=\frac{1}{2}\left\{Y_{j}^{A}, W_{B}^{j}\right\}, \\
{\left[T_{A B}, T^{C D}\right] } & =\delta_{A}^{C} T_{B}^{D}+\delta_{A}^{D} T_{B}^{C}+\delta_{B}^{C} T_{A}^{D}+\delta_{B}^{D} T_{A}^{C}, \\
{\left[T_{A}^{B}, T^{C D}\right] } & =\delta_{A}^{C} T^{B D}+\delta_{A}^{D} T^{B C}, \quad\left[T_{A}^{B}, T_{C D}\right]=-\delta_{C}^{B} T_{A D}-\delta_{D}^{B} T_{A C} .
\end{aligned}
$$


Note that the oscillator representation provides a standard tool for the study of representations of $\mathfrak{s p}(2 M)[31,32]$ (and references therein).

Rank-r equations (1.4) have the form (3.15). The operator $\sigma_{-}^{\mathbf{r}}(1.5)$ obeys

$$
\left(\sigma_{-}^{\mathbf{r}}\right)^{2}=0, \quad\left\{\mathrm{~d}, \sigma_{-}^{\mathbf{r}}\right\}=0
$$

which implies that the corresponding connection is flat.

System (1.4) as well as generators (3.17) are invariant under the action of $\mathfrak{o}(\mathbf{r})$ on the color indices $i, j, \ldots=1, \ldots \mathbf{r}$, that leaves invariant $\delta_{i j}$. Generators of $\mathfrak{o}(\mathbf{r})$

$$
\tau_{m k}=t_{m k}-t_{k m}, \quad t_{m j}=\delta_{j i} t_{m}^{i}, \quad t_{m}^{i}=\frac{1}{2}\left\{Y_{m}^{A}, W_{A}^{i}\right\},
$$

obey the standard commutation relations

$$
\left[\tau_{m p}, \tau_{q j}\right]=\delta_{p q} \tau_{m j}+\delta_{m j} \tau_{p q}-\delta_{m q} \tau_{p j}-\delta_{p j} \tau_{m q}
$$

Being mutually commuting, $\mathfrak{o}(\mathbf{r})$ and $\mathfrak{s p}(2 M)$ form a Howe-dual pair [33].

As in the lower-rank cases, rank- $r$ primary fields obey the condition

$$
\sigma_{-}^{\mathbf{r}} C(Y \mid X)=0 .
$$

In terms of the expansion

$$
C(Y \mid X)=\sum_{n} C_{A_{1} ; \ldots ; A_{n}}^{i_{1} ; \ldots ; i_{n}}(X) Y_{i_{1}}^{A_{1}} \cdots Y_{i_{n}}^{A_{n}}
$$

eq. (3.22) implies tracelessness of the component fields with respect to color indices

$$
\delta_{i_{1} i_{2}} C_{A_{1} ; \ldots ; A_{n}}^{i_{1} ; \ldots ; i_{n}}(X)=0 \quad \forall n \geq 2 .
$$

Since $Y_{i}^{A}$ commute, the tensors $C_{A_{1} ; \ldots ; A_{n}}^{i_{1} ; \ldots ; i_{n}}(X)$ are symmetric with respect to permutation of any pair of upper and lower indices. Hence $C_{A_{1} ; \ldots ; A_{n}}^{i_{1} ; \ldots ; i_{n}}(X)$ can be decomposed into a direct sum of tensors forming irreducible representations of $\mathfrak{o}(\mathbf{r})$ as well as of $\mathfrak{g l}_{M}$ with the same symmetry properties described by some YD $\mathbf{Y}\left(l_{1}, \ldots, l_{m}\right)\left(l_{1}+\ldots+l_{m}=n\right)$. Abusing terminology, a $\mathfrak{g l}(M)$ YD associated with a traceless tensor with respect to color indices will be referred to as traceless YD.

Recall that if a traceless tensor with respect to color indices taking $\mathbf{r}$ values has symmetry of $\mathbf{Y}\left[h_{1}, \ldots, h_{m}\right]$, it can be nonzero only if

$$
\mathrm{h}_{1}(\mathbf{Y})+\mathrm{h}_{2}(\mathbf{Y}) \leq \mathbf{r} .
$$

Equivalently, for a traceless $\mathrm{YD} \mathrm{Y}_{0}(n_{1}, \ldots, n_{m}, \underbrace{1, \ldots, 1}_{q})\left(n_{m} \geq 2\right)$

$$
2 m+q \leq \mathbf{r}
$$

As shown in section 5.2, for any rank-r primary field $C \in H^{0}\left(\sigma_{-}^{\mathbf{r}}\right)$ associated with a YD $\mathbf{Y}_{0}(n_{1}, \ldots, n_{m}, \underbrace{1, \ldots, 1}_{q})$ that obeys (3.26), the left-hand side of the dynamical equation 
has the symmetry properties of the rank-r two-column dual YD $\mathbf{Y}_{1}(n_{1}, \ldots, n_{m}, \underbrace{2, \ldots, 2}_{\mathbf{r}+1-2 m-q}$, $\underbrace{1, \ldots, 1}_{q})(3.14)$.

Pictorially,

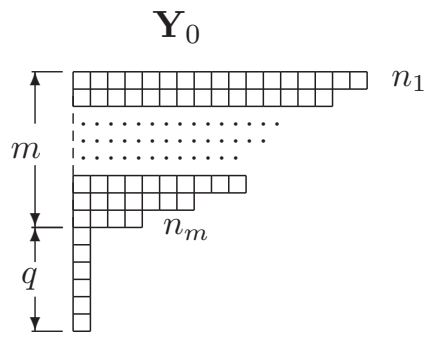

$$
n_{m} \geq 2
$$

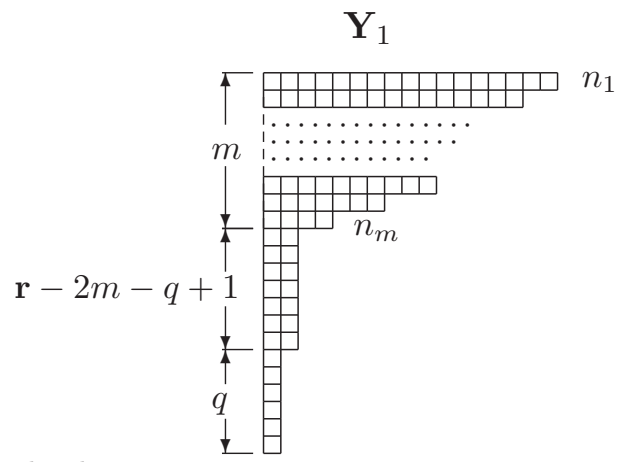

Rank-r two-column duality.

$\mathbf{Y}_{0}$ and $\mathbf{Y}_{1}$ are $\mathfrak{g l}_{M}$-Young diagrams with respect to indices $A, B=1, \ldots, M$. Note that for $M<\mathbf{r}$ some of them may be zero which would mean either that the corresponding primary field is absent $\left(\mathbf{Y}_{0}=0\right)$ or that it does not obey any dynamical equations $\left(\mathbf{Y}_{1}=0\right)$, i.e., the system is off-shell.

Dynamical equations are most conveniently described in terms of Young diagrams with manifest antisymmetrization. For nonnegative integers $h_{1} \geq h_{2}, h_{1}+h_{2} \leq \mathbf{r}$ consider a tensor

$$
\begin{array}{lll}
\mathcal{E}_{i_{1}\left[h_{1}\right],}^{A_{1}\left[\mathbf{r}-h_{2}+1\right],}, A_{2}\left[\mathbf{r}-h_{1}+1\right], & A_{3}\left[h_{3}\right], \ldots, A_{k}\left[h_{n}\right] \\
i_{2}\left[h_{2}\right], & i_{3}\left[h_{3}\right], \ldots, i_{k}\left[h_{n}\right]
\end{array}
$$

with the symmetry $\mathbf{Y}_{0}\left[h_{1}, h_{2}, h_{3}, \ldots, h_{n}\right]$ in the lower indices and $\mathbf{Y}_{1}\left[\mathbf{r}-h_{2}+1, \mathbf{r}-h_{1}+\right.$ $\left.1, h_{3}, \ldots, h_{n}\right]$ in the upper ones. So defined $\mathbf{Y}_{0}$ and $\mathbf{Y}_{1}$ obey two-column duality condition (3.14). Let $\mathcal{E}$, which plays a role of projector to the respective tensor representations, be traceless with respect to the color indices. It is not difficult to see that, for any $\mathcal{E}(3.28)$, the following equations hold as a consequence of (1.4)

$$
\widehat{\mathcal{E}}_{\mathbf{Y}_{0}} \mathcal{C}(Y \mid X)=0
$$

where

$$
\begin{aligned}
& \widehat{\mathcal{E}}_{\mathbf{Y}_{0}}=\mathcal{E}_{i_{1}\left[h_{1}\right],}^{A_{1}\left[\mathbf{r}-h_{2}+1\right],}, A_{2}\left[\mathbf{r}-h_{1}+1\right], A_{2}\left[h_{3}\right], \ldots, A_{n}\left[h_{n}\right]
\end{aligned}
$$

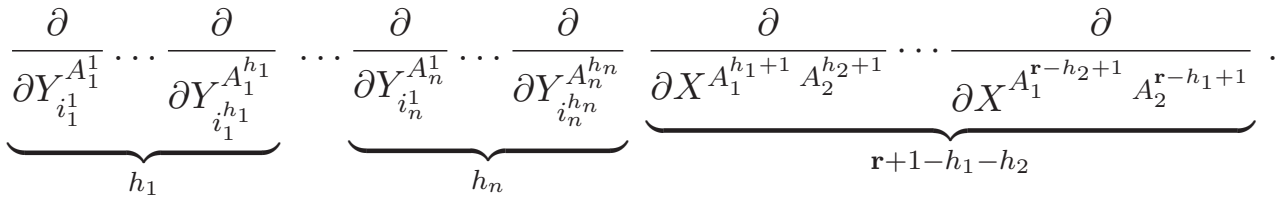


Indeed, by virtue of (1.4), eq. (3.29) is equivalent to

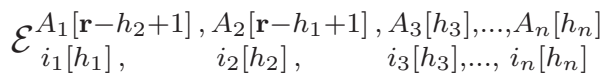

$$
\begin{aligned}
& \delta_{j_{1}^{1} j_{2}^{1}} \cdots \delta_{j_{1}^{N} j_{2}^{N}} \frac{\partial^{2}}{\partial Y_{j_{1}^{1}}^{A_{1}^{h_{1}+1}} \partial Y_{j_{2}^{1}}^{A_{2}^{h_{2}+1}}} \cdots \frac{\partial^{2}}{\partial Y_{j_{1}^{N}}^{A^{\mathbf{r}-h_{1}+1}} \partial Y_{j_{2}^{N}}^{A^{\mathbf{r}-h_{2}+1}}} \\
& \frac{\partial}{\partial Y_{i_{1}^{1}}^{A_{1}^{1}}} \cdots \frac{\partial}{\partial Y_{i_{1}^{h_{1}}}^{A_{1}^{h_{1}}}} \frac{\partial}{\partial Y_{i_{2}^{1}}^{A_{2}^{1}}} \cdots \frac{\partial}{\partial Y_{i_{2}^{h_{2}}}^{A_{2}^{h_{2}}}} \cdots \cdots \frac{\partial}{\partial Y_{i_{n}^{1}}^{A_{n}^{1}}} \cdots \frac{\partial}{\partial Y_{i_{n}^{h_{n}^{n}}}^{A_{n}^{h_{n}}}} \mathcal{C}(Y \mid X)=0,
\end{aligned}
$$

where $N=\mathbf{r}+1-h_{1}-h_{2}$. Since derivatives $\frac{\partial}{\partial Y_{j}^{B}}$ commute, while the indices in columns of the both of the Young diagrams, $\mathbf{Y}\left[h_{1}, h_{2}, h_{3}, \ldots, h\right]$ and its two-column dual $\mathbf{Y}\left[\mathbf{r}-h_{2}+\right.$ $\left.1, \mathbf{r}-h_{1}+1, h_{3}, \ldots, h\right]$, are antisymmetrized, the indices $j_{1}^{1} \ldots j_{1}^{N}$ are antisymmetrized with the indices $i_{1}\left[h_{1}\right]$, while the indices $j_{2}^{1} \ldots j_{2}^{N}$ are antisymmetrized with the indices $i_{2}\left[h_{2}\right]$. Such antisymmetrizations yields zero by virtue of the following

Lemma 1 Let a tensor $\mathcal{F}_{i[m], j[n]}$ be traceless and antisymmetric both in indices $i$ and in $j$ taking $\mathbf{r}$ values $\left(\mathcal{F}_{i[m], j[n]}\right.$ is not demanded to have properties of a Young diagram). Consider the tensor

$$
\left.\left.\mathcal{G}_{i[m+p], j[n+p]}=\mathcal{F}_{[i[m],[j[n]} \delta_{i_{m+1} j_{n+1}} \ldots \delta_{i_{m+p} j_{n+p}}\right]_{j}\right]_{i}
$$

resulting from the total antisymmetrization of indices $i$ and $j$. Then

$$
\mathcal{G}_{i[m+p], j[n+p]}=0 \quad \text { at } \quad m+n>\mathbf{r}-p .
$$

The proof follows from the determinant formula applied to the double dual tensor $\tilde{\mathcal{G}}$

$$
\tilde{\mathcal{G}}^{k[\mathbf{r}-m-p], l[\mathbf{r}-n-p]}:=\epsilon^{k[\mathbf{r}-m-p] i[m+p]} \epsilon^{l[\mathbf{r}-n-p] j[n+p]} \mathcal{G}_{i[m+p], j[n+p]} .
$$

Indeed,

$$
\tilde{\mathcal{G}}^{k[\mathbf{r}-m-p], l[\mathbf{r}-n-p]}:=\epsilon^{k[\mathbf{r}-m-p] i[m]}{ }_{\mathbf{r}[p]} \epsilon^{l[\mathbf{r}-n-p] j[n] \mathbf{r}[p]} \mathcal{F}_{i[m], j[n]} .
$$

Expressing the product of two totally antisymmetric symbols in terms of Kronecker symbols one observes that for $m+n>\mathbf{r}-p$ at least one pair of indices of the traceless tensor $\mathcal{F}_{i[m], j[n]}$ is contracted hence giving zero.

Note that eq. (3.33) at $p=0$ yields eq. (3.25).

Since the differential operator $\widehat{\mathcal{E}}_{\mathbf{Y}_{0}}(3.30)$ is homogeneous, the primary fields also satisfy (3.29). The parameter (3.28) is designed in such a way that eq. (3.29) is nontrivial only for primaries $C(Y \mid X)$ with the symmetry properties of $\mathbf{Y}_{0}\left[h_{1}, \ldots, h_{n}\right]$. The nontrivial part of the story is to prove that the presented list of dynamical equations is complete. This follows from the analysis of the cohomology group $H^{1}\left(\sigma_{-}^{\mathbf{r}}\right)$ in section 5 .

As anticipated, there is precise matching between the primaries and field equations. This means that the respective subspaces of $H^{0}\left(\sigma_{-}^{\mathbf{r}}\right)$ and $H^{1}\left(\sigma_{-}^{\mathbf{r}}\right)$ form isomorphic $\mathfrak{o}(\mathbf{r})$ modules. Here one should not be confused by the fact that the two-column dual diagram $\mathbf{Y}_{1}$ does not respect condition (3.25). The point is that, as an $\mathfrak{o}(\mathbf{r})$-tensor, $\mathbf{Y}_{1}$ is not 
traceless, containing explicitly a number of $\mathfrak{o}(\mathbf{r})$ metric tensors $\delta_{i j}$ which add additional $\mathfrak{o}(\mathbf{r})$ indices to $\mathbf{Y}_{1}$ compared to $\mathbf{Y}_{0}$.

The particular case of (3.29) which plays the key role in the construction of conserved currents is that with $\mathbf{r}=2 \kappa$ and a traceless $\mathfrak{o}(2 \kappa)$-diagram $\mathbf{Y}_{0}=\mathbf{Y}_{0}^{2 \kappa_{\text {cur }}}$ of the form

$$
\mathbf{Y}_{0}^{2 \kappa_{\text {cur }}}\left[h_{1}, h_{2}, h_{3}, h_{4}, \ldots\right]=\mathbf{Y}_{0}^{2 \kappa_{\text {cur }}}\left[\kappa, \kappa, h_{3}, h_{4}, \ldots\right] .
$$

Then operator $\widehat{\mathcal{E}}_{\mathbf{Y}_{0}^{2 \kappa_{c u r}}}(3.30)$ is of the first order in $\frac{\partial}{\partial X}$. Hence, eq. (3.29) acquires the form of conservation condition

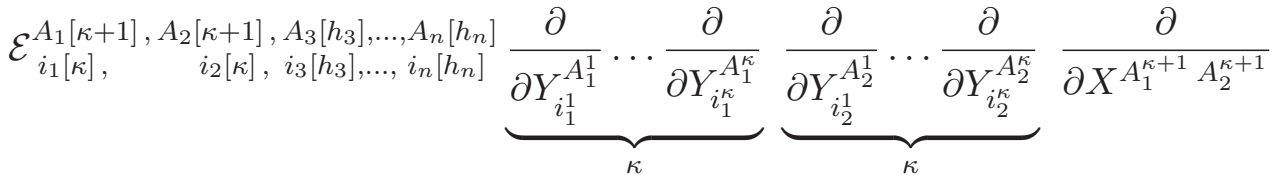

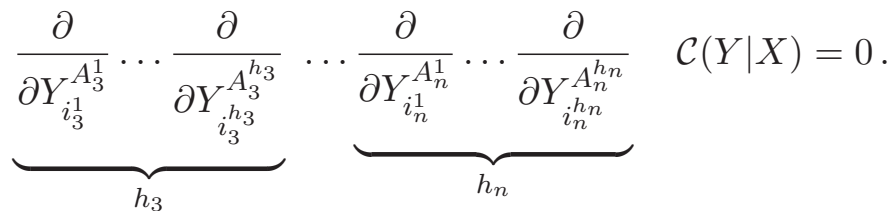

For instance, in the particular case of two-column diagrams this yields a straightforward generalization of the conservation condition of $[6]$

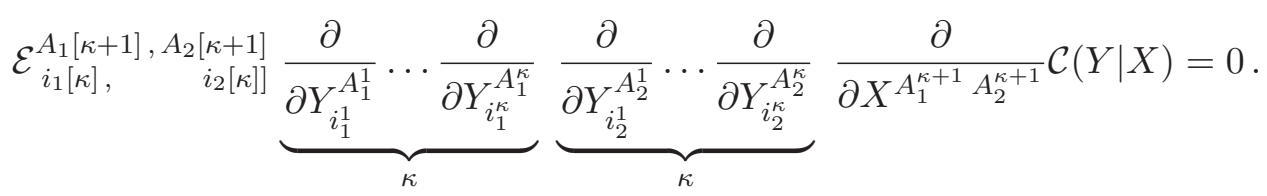

\subsection{Higher $\sigma_{-}^{\mathrm{r}}$ - cohomology in $\mathcal{M}_{M}$}

As shown in [29] (for more detail see [5, 30]), when the fields $\mathcal{C}(Y \mid X)$ satisfying (1.4) are $p$ forms, dynamical fields and their field equations are associated with $H^{p}\left(\sigma_{-}\right)$and $H^{p+1}\left(\sigma_{-}\right)$, respectively. Recall that $H^{p}\left(\sigma_{-}^{\mathbf{r}}\right)=\operatorname{ker}\left(\sigma_{-}^{\mathbf{r}}\right) /\left.\operatorname{Im}\left(\sigma_{-}^{\mathbf{r}}\right)\right|_{p}$, where $\left.\right|_{p}$ denotes the restriction to $p$-forms. Hence, we are interested in $\sigma_{-}^{\mathbf{r}}$-closed $p$-forms $P(\xi, Y \mid X)$,

$$
\sigma_{-}^{\mathbf{r}} P(\xi, Y \mid X)=0
$$

that are not $\sigma_{-}^{\mathbf{r}}$-exact.

In the expansion

$$
P(\xi, Y \mid X)=\sum_{n} C_{A_{1} ; \ldots ; A_{n} \mid C_{1} ; \ldots ; C_{2 p}}^{i_{1} ; \ldots i_{n}}(X) Y_{i_{1}}^{A_{1}} \cdots Y_{i_{n}}^{A_{n}} \xi^{C_{1} C_{2}} \cdots \xi^{C_{2 p-1} C_{2 p}}
$$

the coefficients $C_{A_{1} ; \ldots ; A_{n} \mid C_{1} ; \ldots ; C_{2 p}}^{i_{1} ; \ldots ; i_{n}}(X)$ form almost symmetric diagrams (2.8) with respect the indices $C_{1} ; \ldots ; C_{2 p}$ contracted with the differentials $\xi^{A B}$ and are symmetric with respect to permutation of any pair of upper and lower indices $A_{k}, i_{k}$. Hence, with respect to the latter indices, $C_{A_{1} ; \ldots ; A_{n} \mid C_{1} ; \ldots ; C_{2 p}}^{i_{1} ; \ldots ; i_{n}}$ can be decomposed into a direct sum of tensors forming irreducible representations of $\mathfrak{o}(\mathbf{r})$ as well as of $\mathfrak{g l}_{M}$ with the same symmetry properties described by some YD $\mathbf{Y}^{\prime}$. 


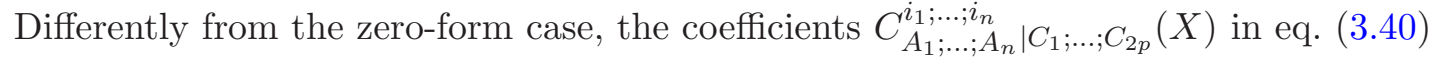
are not necessarily traceless with respect to color indices. In addition to the indices of a $\mathfrak{o}(\mathbf{r})$-traceless $\mathrm{YD}$, the diagram $\mathbf{Y}^{\prime}$ can contain indices carried by products of $\delta^{i j}$. The respective $\mathfrak{g l}(M)$ tensor contains products of the "tracefull" combinations

$$
U^{A B}=\delta^{i j} Y_{i}^{A} Y_{j}^{B},
$$

that, as mentioned in section 2, are described by the Kronecker diagrams (2.6).

As a result, $P(\xi, Y \mid X)$ satisfying (2.6) has symmetry properties that described by direct sum of the following tensor products of YD

$$
\mathbf{Y}_{0}\left[h_{1}, h_{2}, \ldots\right] \otimes \mathbf{Y}_{A}\left[\mathfrak{h}_{1}, \ldots, \mathfrak{h}_{m}\right] \otimes \mathbf{Y}_{\delta}\left[d_{1}, \ldots, d_{2 n}\right]
$$

for different $m, n$ such that $\left|\mathbf{Y}_{A}\right|=2 p$.

Analysis of the higher cohomology with $p>1$ is also interesting in many respects. For instance, it characterizes further relations on the left-hand sides of field equations as well as gauge fields in higher-spin gauge theories, described by higher differential forms. In this section we present the final results for $H^{p}\left(\sigma_{-}^{\mathbf{r}}\right)$ leaving details of their derivation to section 5 and appendix A.

$H^{p}\left(\sigma_{-}^{\mathbf{r}}\right)$ is realized by a space of homogeneous polynomials $P(\xi, Y)$ of degree $p$ in $\xi$, forming an $\mathfrak{o}(\mathbf{r})$-module with respect to the color indices $i$ carried by $Y_{i}^{A}$ and a $\mathfrak{g l}_{M^{-}}$ module with respect to the spinor indices $A, B, \ldots$ carried by $Y_{i}^{A}$ and $\xi^{A B}$. To avoid the restriction that the maximal height of $\mathfrak{g l}_{M^{-}}$Young diagrams is $M$, we will assume that $M$ is large enough. In the final result one should simply take into account that such diagrams are zero. On the other hand, the rank $\mathbf{r}$ is not supposed to be large because nontrivial cohomology is just associated with special values of $\mathbf{r}$.

\subsubsection{Main results}

It turns out that the cohomology groups $H^{p}\left(\sigma_{-}^{\mathbf{r}}\right)$ are characterized by various $\mathfrak{o}(\mathbf{r})$-modules and $\mathfrak{g l}_{M^{-}}$-modules formed by the differentials $\xi^{A B}$. The former can be described by a traceless $\mathfrak{o}(\mathbf{r})$-diagram $\mathbf{Y}_{0}$ while the latter by an almost symmetric $\mathfrak{g l}_{M}$-diagram $\mathbf{Y}_{A}$. Given $\mathbf{Y}_{0}$ and $\mathbf{Y}_{A}$, there exists some tensor with the symmetry of a Kronecker YD $\mathbf{Y}_{\delta}$ (2.6), composed of the Kronecker symbols $\delta^{i j}$, and some component $\mathbf{Y}^{\prime} \in \mathbf{Y}_{0} \otimes \mathbf{Y}_{\delta} \otimes \mathbf{Y}_{A}$ that represents $H^{p}\left(\sigma_{-}^{\mathbf{r}}\right)$.

To identify $\mathbf{Y}^{\prime}$ it is convenient to introduce the infinite almost symmetric matrix $\mathrm{S}=$ $\mathrm{S}\left(\mathbf{r} \mid \mathbf{Y}_{0}\right)$, that has the form

$$
\mathrm{S}=\left(\begin{array}{ccccccccc}
\triangle_{1}^{0} & \Delta_{1}^{0} & \Delta_{2} & \Delta_{3} & \Delta_{4} & \Delta_{5} & \Delta_{6} & \Delta_{7} & \ldots \\
\triangle_{2} & \Delta_{1} & \Delta_{1} & \Delta_{2} & \Delta_{3} & \Delta_{4} & \Delta_{5} & \Delta_{6} & \ldots \\
\triangle_{3} & \Delta_{2} & \Delta_{1}^{0} & \Delta_{1}^{0} & \Delta_{2} & \Delta_{3} & \Delta_{4} & \Delta_{5} & \ldots \\
\triangle_{4} & \Delta_{3} & \Delta_{2} & \Delta_{1} & \Delta_{1} & \Delta_{2} & \Delta_{3} & \Delta_{4} & \ldots \\
\triangle_{5} & \Delta_{4} & \Delta_{3} & \Delta_{2} & \Delta_{1}^{0} & \Delta_{1}^{0} & \Delta_{2} & \Delta_{3} & \ldots \\
\triangle_{6} & \Delta_{5} & \Delta_{4} & \Delta_{3} & \Delta_{2} & \Delta_{1} & \Delta_{1} & \Delta_{2} & \ldots \\
\triangle_{7} & \Delta_{6} & \Delta_{5} & \Delta_{4} & \Delta_{3} & \Delta_{2} & \Delta_{1}^{0} & \Delta_{1}^{0} & \ldots \\
\vdots & \vdots & \vdots & \vdots & \vdots & \vdots & \vdots & \vdots & \ddots
\end{array}\right)
$$


where

$$
\triangle_{k}=\mathrm{h}_{k}\left(\mathbf{Y}_{0}\right)-\mathrm{h}_{k+1}\left(\mathbf{Y}_{0}\right)
$$

are the Cartan weights of $\mathbf{Y}_{0}$ and

$$
\triangle_{1}^{0}=\mathbf{r}-\mathrm{h}_{1}\left(\mathbf{Y}_{0}\right)-\mathrm{h}_{2}\left(\mathbf{Y}_{0}\right) .
$$

Note that here all zero-height columns of $\mathbf{Y}_{0}$ are in the game, i.e., $\mathbf{Y}_{0}$ is treated as a generalized Young diagram with an infinite number of columns.

Elements of matrix (3.43) obey

$$
\begin{array}{rlrl}
\mathrm{S}_{n(n+j)} & =\mathrm{S}_{(n+j-1) n} & & \forall n \geq 1, \quad j \geq 1, \\
\mathrm{~S}_{n m} & =\triangle_{m-n} & & \forall m-n \geq 2, \\
\mathrm{~S}_{(2 k+1)(2 k+1)} & =\triangle_{1}^{0}, & & \\
\mathrm{~S}_{(2 k)(2 k)} & =\triangle_{1} . &
\end{array}
$$

For any almost symmetric $\mathbf{Y}_{A}$ we introduce the shift matrix $\mathbb{S}^{s h}=\mathbb{S}^{s h}\left(\mathbf{r} \mid \mathbf{Y}_{A}, \mathbf{Y}_{0}\right)$ resulting from the intersection of the infinite almost symmetric matrix $\mathrm{S}\left(\mathbf{r} \mid \mathbf{Y}_{0}\right)$ (3.43) with $\mathbf{Y}_{A}$. In other words, elements of the shift matrix $\mathbb{S}^{s h}$ are

$$
\mathbb{S}_{n m}^{s h}=\mathrm{S}_{n m} \theta\left(\mathrm{h}_{m}\left(\mathbf{Y}_{A}\right)-n\right),
$$

where $\theta(n)=0$ if $n<0, \theta(n)=1$ if $n \geq 0$. For example, for $\mathbf{Y}_{A}=\mathbb{Y}^{\text {nest }}\{8,5,3\}(2.12)$, the shift matrix $\mathbb{S}^{s h}\left(\mathbf{r} \mid \mathbb{Y}^{\text {nest }}\{8,5,3\}, \mathbf{Y}_{0}\right)$ is

$$
\mathbb{S}^{s h}=\left(\begin{array}{ccccccccc}
\triangle_{1}^{0} & \Delta_{1}^{0} & \Delta_{2} & \Delta_{3} & \Delta_{4} & \Delta_{5} & \Delta_{6} & \Delta_{7} & \Delta_{8} \\
\triangle_{2} & \Delta_{1} & \Delta_{1} & \Delta_{2} & \Delta_{3} & \Delta_{4} & \Delta_{5} & 0 & 0 \\
\triangle_{3} & \Delta_{2} & \Delta_{1}^{0} & \Delta_{1}^{0} & \Delta_{2} & \Delta_{3} & 0 & 0 & 0 \\
\triangle_{4} & \Delta_{3} & \Delta_{2} & 0 & 0 & 0 & 0 & 0 & 0 \\
\triangle_{5} & \Delta_{4} & \Delta_{3} & 0 & 0 & 0 & 0 & 0 & 0 \\
\triangle_{6} & \Delta_{5} & 0 & 0 & 0 & 0 & 0 & 0 & 0 \\
\triangle_{7} & 0 & 0 & 0 & 0 & 0 & 0 & 0 & 0 \\
\triangle_{8} & 0 & 0 & 0 & 0 & 0 & 0 & 0 & 0
\end{array}\right) .
$$

The central result is

Theorem 1 The $\mathfrak{g l}(M)$-Young diagrams associated with $H^{p}\left(\sigma_{-}^{\mathbf{r}}\right)$ are

$$
\mathbf{Y}^{\prime}=\mathbf{Y}^{\prime}\left(p|\mathbf{r}| \mathbf{Y}_{A}, \mathbf{Y}_{0}\right)
$$

where $\mathbf{Y}_{A}$ is any almost symmetric diagram, $\left|Y_{A}\right|=2 p, \mathbf{Y}_{0}$ is any traceless diagram,

$$
\mathrm{h}_{j}\left(\mathbf{Y}^{\prime}\right)=\mathcal{H}_{j}+\mathfrak{h}_{j}, \quad \mathcal{H}_{j}=h_{j}+\sum_{i} \mathbb{S}_{i j}^{s h}, \quad \mathfrak{h}_{j}=\mathrm{h}_{j}\left(\mathbf{Y}_{A}\right), \quad h_{j}=\mathrm{h}_{j}\left(\mathbf{Y}_{0}\right)
$$

and $\mathbb{S}_{i j}^{s h}$ are elements of the shift matrix $\mathbb{S}^{s h}\left(\mathbf{r} \mid \mathbf{Y}_{A}, \mathbf{Y}_{0}\right)$ (3.47). 
The proof of Theorem 1 is given in appendix A using the homotopy trick explained in section 5 .

As shown in appendix, the Kronecker $\mathrm{YD} \mathrm{Y}_{\delta}\left[d_{1}, d_{1}, \ldots, d_{\mathbf{m}}, d_{\mathbf{m}}\right]$ can be expressed via $\mathcal{H}_{j}(3.50)$ as follows

$$
d_{k}=\mathcal{H}_{2 k}+\mathcal{H}_{2 k-1}-\mathbf{r} \quad \forall k \leq \mathbf{m}=\left[\frac{1}{2}(\mathbf{n}+1)\right], \quad \mathbf{n}=\sharp\left(\mathbf{Y}_{A}\right) .
$$

$\left([a] \leq a\right.$ denotes the integer part of $a$.) For example, for the nested hook $\mathbf{Y}_{A}(2.12) \mathbf{m}=2$ and $d_{1}=\mathbf{r}-h_{6}-h_{9}, d_{2}=\mathbf{r}-h_{1}-h_{4}$.

By eqs. (3.50), (3.51) the nonzero heights of the Kronecker YD $\mathbf{Y}_{\delta}$ are

$$
\mathrm{h}_{2 j-1}\left(\mathbf{Y}_{\delta}\right)=\mathrm{h}_{2 j}\left(\mathbf{Y}_{\delta}\right)=h_{2 j-1}+\sum_{i} \mathbb{S}_{i(2 j-1)}^{s h}+h_{2 j}+\sum_{i} \mathbb{S}_{i(2 j)}^{s h}-\mathbf{r}, \quad j \leq \mathbf{m}
$$

For the case of one-forms described by the simplest nested hook $\mathbf{Y}_{A}=\mathbf{Y}[1,1]$ this gives $\mathbb{S}_{11}^{s h}=\mathbb{S}_{12}^{s h}=\mathbf{r}-h_{1}-h_{2}=d_{1}$. Hence for $H^{1}\left(\sigma_{-}^{\mathbf{r}}\right)$ eq. (3.49) yields $\mathrm{h}_{1}\left(\mathbf{Y}^{\prime}\right)=\mathbf{r}+1-h_{2}$, $\mathrm{h}_{2}\left(\mathbf{Y}^{\prime}\right)=\mathbf{r}+1-h_{1}$. In accordance with section 3.1.2 and definition (3.14), $\mathbf{Y}^{\prime}$ is two-column dual to $\mathbf{Y}_{0}$.

\subsection{Multilinear currents in $\mathcal{M}_{M}$}

The construction of conserved currents in terms of closed forms proposed in $[5,6]$ for rank-one fields admits a higher-rank generalization.

That dynamical degrees of freedom associated with the rank-one equations (1.1) live on a $M$-dimensional surface $S \subset \mathcal{M}_{M} \otimes \mathbb{R}^{M}$ suggests that conserved charges associated with these equations have to be built in terms of $M$-forms that are closed as a consequence of rank-two field equations (1.2). As shown in [35], the following $M$-form in $\mathcal{M}_{M} \otimes \mathbb{R}^{2 M}$

$$
\left.\left(\xi^{A B} \frac{\partial}{\partial z^{B}}-d \bar{z}^{A}\right)^{M} J^{2}(z, \bar{z} \mid X)\right|_{z=0}
$$

is closed provided that $J^{2}(z, \bar{z} \mid X)$ solves (1.2) with $z, \bar{z}(3.8)$. Usual conserved currents

$$
J_{\eta}^{2}(z, \bar{z} \mid X)=\eta \delta^{k j} \mathcal{C}_{k}(z+\bar{z} \mid X) \mathcal{C}_{j}(i(\bar{z}-z) \mid X)
$$

are built in terms of bilinears in solutions $\mathcal{C}_{j}$ of (1.1) with parameters $\eta$ that commute with $\sigma_{-}^{2}(1.3)[5,10,26]$.

Since modules of solutions of the rank-r equations in $\mathcal{M}_{M} \otimes \mathbb{R}^{\mathbf{r} M}$ are functions of $\mathbf{r} M$ variables $Y_{i}^{A}$ one might guess that in the rank-r case the dimension of a "local Cauchy bundle" [7] on which initial data should be given to determine a solution everywhere in $\mathcal{M}_{M}$ is $\mathbf{r} M$. One can see however that the following straightforward generalization of (3.53) to conserved currents in $\mathcal{M}_{M} \otimes \mathbb{R}^{2 \mathbf{r} M}$ with arbitrary $\mathbf{r}$

$$
\left.\prod_{j=1}^{\mathbf{r}}\left(\xi^{A^{j} B} \frac{\partial}{\partial z_{j}^{B}}-d \bar{z}_{j}{ }^{A^{j}}\right)^{M} J_{\eta}^{2 \mathbf{r}}(z, \bar{z} \mid X)\right|_{z=0},
$$


where $Y_{j}=z_{j}+\bar{z}_{j}, Y_{j+\mathbf{r}}=i\left(\bar{z}_{j}-z_{j}\right), \varepsilon_{A_{1} \ldots A_{M}}$ is the rank-M Levi-Civita symbol, and

$$
J_{\eta}^{2 \mathbf{r}}(z, \bar{z} \mid X)=\eta \delta^{k j} \mathcal{C}_{k}^{\mathbf{r}}(z+\bar{z} \mid X) \mathcal{C}_{j}^{\mathbf{r}}(i(\bar{z}-z) \mid X)
$$

is bilinear in rank-r fields $\mathcal{C}_{i}^{\mathbf{r}}$, does not work. The reason is that, although these forms are closed by virtue of the rank-2r unfolded equations, their pullback to $\mathcal{M}_{M}$ is zero for $\mathbf{r}>1$ thus obstructing the construction of conserved charges in the form of integrals over $\mathcal{M}_{M}$.

Indeed, up to a numerical factor, the pullback of the form (3.54) to $\mathcal{M}_{M}$ is

$$
\left.\left\{\prod_{j=1}^{\mathbf{r}} \varepsilon_{A_{1}^{j}, \ldots, A_{M}^{j}} \xi^{A_{1}^{j} B_{1}^{j}} \ldots \xi^{A_{M}^{j} B_{M}^{j}}\right\}\left(\frac{\partial}{\partial z_{j}^{B_{1}^{j}}} \ldots \frac{\partial}{\partial z_{j}^{B_{M}^{j}}} J^{2 \mathbf{r}}(z, \bar{z} \mid X)\right)\right|_{z=\bar{z}=0} .
$$

Any Young diagram associated with the combination of differentials $\xi^{A B}$ can only contain rows of lengths $l \leq M+1$ since otherwise it would contain symmetrization of a pair of differentials. On the other hand, because of $\varepsilon$-symbols, it contains $\mathbf{r}$ columns of the maximal height $M$ associated with the indices $A$ in (3.56). Anticommutativity of the differentials implies that the part of the diagram associated with the indices $B$ should contain $\mathbf{r}$ total symmetrizations. However, since the first $\mathbf{r}$ columns are occupied by the indices $A$ and the total number of symmetrized indices cannot exceed $M+1$, this is not possible for $\mathbf{r}>1$ as at least two symmetrized indices $B$ would belong to the same column, which implies antisymmetrization. Thus (3.56) is zero for $\mathbf{r}>1$.

The reason why the naive construction does not work is that the $\mathfrak{o}(\mathbf{r})$, that acts on the primary (dynamical) rank-r fields, relates different solutions to each other not affecting evolution of any given solution. This suggests that the dimension of the true local Cauchy bundle is $\mathbf{r} M-\frac{1}{2} \mathbf{r}(\mathbf{r}-1)$. Correspondingly, the respective currents should be represented by closed $\mathbf{r}\left(M-\frac{1}{2}(\mathbf{r}-1)\right)$-forms.

As shown in section 2, products of anticommuting variables $\xi^{A B}$ have symmetries of almost symmetric Young diagrams. The product of $\mathbf{r}$ totally antisymmetric tensors with $1 \leq \mathbf{r}<M$ admits a unique extension to such a tensor with $(2 \mathbf{r} M-\mathbf{r}(\mathbf{r}-1))$ cells described by the YD $\mathbf{Y}[\underbrace{M, \ldots, M}_{\mathbf{r}}, \underbrace{\mathbf{r}, \ldots, \mathbf{r}}_{M+1-\mathbf{r}}]$. Pictorially,

$$
\mathbf{Y}[\underbrace{M, \ldots, M}_{\mathbf{r}}, \underbrace{\mathbf{r}, \ldots, \mathbf{r}}_{M+1-\mathbf{r}}]=M \prod_{\mathbf{r}}^{M+1} \mathbf{r}
$$

Setting $N=M+1-\mathbf{r}$ consider the differential form

$$
\begin{aligned}
& \Xi^{D_{1}[M], \ldots, D_{\mathbf{r}}[M], D_{\mathbf{r}+1}[\mathbf{r}], \ldots, D_{M+1}[\mathbf{r}]}=\xi^{D_{1}^{1} D_{2}^{1}} \xi^{D_{1}^{2} D_{3}^{1}} \ldots \xi^{D_{1}^{\mathbf{r}-1} D_{\mathbf{r}}^{1}} \xi^{D_{1}^{\mathbf{r}} A_{1}^{1}} \ldots \xi^{D_{1}^{M} A_{N}^{1}} \ldots \\
& \xi^{D_{n}^{n} D_{n+1}^{n}} \xi^{D_{n}^{n+1} D_{n+2}^{n}} \ldots \xi^{D_{n}^{\mathbf{r}-1} D_{\mathbf{r}}^{n}} \xi^{D_{n}^{\mathbf{r}} A_{1}^{n}} \ldots \xi^{D_{n}^{M} A_{N}^{n}} \ldots \\
& \xi^{D_{\mathbf{r}}^{\mathbf{r}} A_{1}^{\mathrm{r}}} \xi^{D_{\mathbf{r}}^{\mathbf{r}+1} A_{2}^{\mathrm{r}}} \ldots \xi^{D_{\mathbf{r}}^{M} A_{N}^{\mathbf{r}}}
\end{aligned}
$$

which by construction has symmetry of $\mathbf{Y}[\underbrace{M, \ldots, M}_{\mathbf{r}}, \underbrace{\mathbf{r}, \ldots, \mathbf{r}}_{N}](3.57)$. Contracting indices of the first $\mathbf{r}$ columns with the totally antisymmetric symbols $\epsilon_{A_{1} \ldots A_{\mathbf{r}}}$ yields a tensor $\mathcal{B}$ of 
the symmetry of $\mathbf{Y}[\underbrace{\mathbf{r}, \ldots \mathbf{r}}_{N}]$

$$
\mathcal{B}^{A_{1}[\mathbf{r}], \ldots, D_{N}[\mathbf{r}]}=\epsilon_{D_{1}^{1} \ldots D_{1}^{M}} \ldots \epsilon_{D_{\mathbf{r}}^{1} \ldots D_{\mathbf{r}}^{M}} \Xi^{D_{1}[M], \ldots, D_{\mathbf{r}}[M], A_{1}[\mathbf{r}], \ldots, A_{N}[\mathbf{r}]} .
$$

Let a tensor $\mathcal{F}_{i_{1}[\mathbf{r}], i_{2}[\mathbf{r}], \ldots, i_{N}[\mathbf{r}]}$ be traceless with respect to the color indices and have the symmetry of $\mathbf{Y}[\underbrace{\mathbf{r}, \ldots \mathbf{r}}_{N}]$. Then the $\left(\mathbf{r} M-\frac{\mathbf{r}(\mathbf{r}-1)}{2}\right)$-form

$$
\begin{aligned}
\Omega_{2 \mathbf{r}}(J)= & \mathcal{B}^{A_{1}[\mathbf{r}], A_{2}[\mathbf{r}], \ldots, A_{N}[\mathbf{r}]} \mathcal{F}_{i_{1}[\mathbf{r}], i_{2}[\mathbf{r}], \ldots, i_{N}[\mathbf{r}]} \\
& \left.\underbrace{\frac{\partial}{\partial Y_{i_{1}^{1}}^{A_{1}^{1}}} \ldots \frac{\partial}{\partial Y_{i_{1}^{\mathrm{r}}}^{A_{1}^{\mathrm{r}}}}}_{\mathbf{r}} \cdots \underbrace{\frac{\partial}{\partial Y_{i_{N}^{1}}^{A_{1}^{1}}} \cdots \frac{\partial}{\partial Y_{i_{N}^{\mathrm{r}}}^{A_{N}^{\mathbf{r}}}}}_{\mathbf{r}} J^{2 \mathbf{r}}(Y \mid X)\right|_{Y=0}
\end{aligned}
$$

is closed provided that a rank-2r field $J^{2 \mathbf{r}}$ (current) obeys the rank-2r current equation

$$
\left(\frac{\partial}{\partial X^{A B}}+\frac{\partial^{2}}{\partial Y_{k}^{A} \partial Y_{j}^{B}} \delta_{k j}\right) J^{2 \mathbf{r}}(Y \mid X)=0 \quad(k, j=1, \ldots, 2 \mathbf{r}),
$$

that coincides with unfolded equations (1.4) with $\mathbf{r}$ replaced by $2 \mathbf{r}$.

Indeed, consider a $\left(\mathbf{r} M-\frac{\mathbf{r}(\mathbf{r}-1)}{2}\right)$-form

$$
\Omega_{2 \mathbf{r}}(\mathrm{A})=\Xi^{D_{1}[M], \ldots, D_{\mathbf{r}}[M], B_{1}[\mathbf{r}], \ldots, B_{N}[\mathbf{r}]} \mathrm{A}_{D_{1}[M], \ldots, D_{\mathbf{r}}[M], B_{1}[\mathbf{r}], B_{N}[\mathbf{r}]}(X),
$$

where A has symmetry of almost symmetric YD (3.57). Then the only non-zero component of the differential form $d \Omega_{2 \mathbf{r}}(\mathrm{A})=\xi^{A B} \frac{\partial}{\partial X^{A B}} \Omega_{2 \mathbf{r}}(\mathrm{A})$ is represented by the almost symmetric Young diagram

$$
\mathbf{Y}[\underbrace{M, \ldots, M}_{\mathbf{r}}, \mathbf{r}+1, \mathbf{r}+1, \underbrace{\mathbf{r}, \ldots, \mathbf{r}}_{N-2}]=
$$

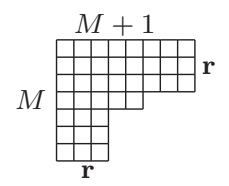

because the first column of $\mathbf{Y}[\underbrace{M, \ldots, M}_{\mathbf{r}}, \underbrace{\mathbf{r}, \ldots, \mathbf{r}}_{N}](3.57)$ is of the maximal height and cannot be enlarged further.

However, eq. (3.37) associated with the traceless $\mathfrak{o}(2 \mathbf{r})$-diagram $\mathbf{Y}_{0}^{2 \mathbf{r}_{\text {cur }}}=$ $\mathbf{Y}_{0}^{2 \mathbf{r}_{\text {cur }}}[\mathbf{r}, \mathbf{r}, \ldots]$ (3.36), which is the first-order differential equation with respect to $X$ derivatives, just implies that the projection to this diagram is zero. Hence, the form $\Omega_{2 \mathbf{r}}(J)(3.59)$ is closed on shell.

In particular, closed form (3.59) with

$$
J^{2 \mathbf{r}}(Y \mid X) \sim \mathcal{C}_{1}\left(Y_{1} \mid X\right) \ldots \mathcal{C}_{2 \mathbf{r}}\left(Y_{2 \mathbf{r}} \mid X\right)
$$

where $\mathcal{C}_{j}\left(Y_{j} \mid X\right)$ solve rank-one equations (1.4), generates $2 \mathbf{r}$-linear conserved currents. As in the case of bilinear currents [10], the first-order differential operators $\mathcal{A}^{1}(Y \mid X)$ and $\mathcal{A}^{2}(Y \mid X)$

$$
\mathcal{A}_{j}^{1 B}\left(Y_{j} \mid X\right)=2 X^{A B} \frac{\partial}{\partial Y_{j}^{A}}-Y_{j}^{B}, \quad \mathcal{A}_{j C}^{2}\left(Y_{j} \mid X\right)=\frac{\partial}{\partial Y_{j}^{C}}, \quad j=1, \ldots, 2 \mathbf{r}
$$


as well as any polynomial $\eta(\mathcal{A})$, obey

$$
\left[\left(\frac{\partial}{\partial X^{A B}}+\frac{\partial^{2}}{\partial Y_{k}^{A} \partial Y_{j}^{B}} \delta_{k j}\right), \eta(\mathcal{A})\right]=0 \quad(k, j=1, \ldots, 2 \mathbf{r}) .
$$

Therefore, for any $\eta^{j_{1}, \ldots, j_{2} \mathrm{r}}(\mathcal{A})$,

$$
J_{\eta}(Y \mid X)=\eta^{j_{1}, \ldots, j_{2 \mathbf{r}}}(\mathcal{A}) \mathcal{C}_{j_{1}}\left(Y_{1} \mid X\right) \ldots \mathcal{C}_{j_{2 \mathbf{r}}}\left(Y_{2 \mathbf{r}} \mid X\right),
$$

also obeys current equation (3.60), giving rise to the $2 \mathbf{r}$-linear charges $Q_{\eta}^{2 \mathbf{r}}$ where $\eta^{j_{1}, \ldots, j_{2 \mathbf{r}}}(\mathcal{A})$ are parameters of the symmetries generated by these multilinear charges, which are analogous to the multiparticle symmetries considered in [36].

\section{Minkowski-like reduction}

\subsection{Fields and equations in $\mathcal{M}_{M}^{M n k}$}

The unfolded form of the equations of motion for massless fields of all spins in $4 d$ Minkowski space (1.6) is a subsystem of (1.4) at $M=4, \mathbf{r}=2$ and $A=\left(\alpha, \alpha^{\prime}\right), B=\left(\beta, \beta^{\prime}\right)$ etc, i.e.,

$$
Y^{A}=\left(y^{\alpha}, \bar{y}^{\beta^{\prime}}\right), \quad X^{A B}=\left(x^{\alpha \beta^{\prime}}, x^{\alpha \beta}, \bar{x}^{\alpha^{\prime} \beta^{\prime}}\right) .
$$

More generally, consider $X^{A B}(4.1)$ with $\alpha=1, \ldots, K$ and $\alpha^{\prime}=1, \ldots, K$ for any $K$. The rank-r generalization (1.8) of (1.6), is a subsystem of (1.4) with $M=2 K$, We set $\eta^{k j}=\delta^{k j}$ in (1.8), setting

$$
\sigma_{-}^{\mathbf{r} M n k}=i \xi^{\alpha \beta^{\prime}} \frac{\partial}{\partial y_{k}^{\alpha}} \frac{\partial}{\partial \bar{y}^{\beta^{\prime} k}}, \quad \bar{y}^{\beta^{\prime} k}=\bar{y}_{j}^{\beta^{\prime}} \delta^{k j} .
$$

Zero-form primary fields in $\mathcal{M}_{M}^{M n k}$

$$
C(y, \bar{y} \mid x)=\sum_{p, q} C_{\alpha_{1} ; \ldots ; \alpha_{p} ; \alpha_{1}^{\prime} ; \ldots ; \alpha^{\prime}{ }^{\prime}}^{i_{1} ; \ldots ; i_{p} ; j_{1} ; \ldots ; j_{q}}(x) y_{i_{1}}^{\alpha_{1}} \ldots y_{i_{p}}^{\alpha_{p}} \bar{y}_{j_{1}}^{\alpha^{\prime}{ }_{1}} \ldots \bar{y}_{j_{q}{ }^{\alpha^{\prime}}}^{\alpha^{\prime}}
$$

satisfy the mutual tracelessness condition with respect to color indices

$$
\sigma_{-}^{\mathbf{r} M n k} C(y, \bar{y} \mid x)=0,
$$

which implies

$$
\delta_{m n} C_{\alpha_{1} ; \alpha_{2} ; \ldots ; \alpha_{p} ; \alpha^{\prime} ; 1_{1} ; \alpha_{2}^{\prime} ; \ldots ; \alpha^{\prime}{ }^{\prime} q}^{m ; i_{2} ; \ldots ; i_{p} ; n ; j_{2} ; \ldots ; j_{q}}=0 .
$$

In these terms, the algebra $\mathfrak{o}(\mathbf{r})$ extends to $\mathfrak{u}(\mathbf{r})$ acting on the conjugated representations carried by lower and upper color indices, while $\mathfrak{s p}(2 M)$ reduces to $\mathfrak{u}(K, K)$ that acts on spinor indices and commutes with the $\mathfrak{u}(\mathbf{r})$. Note that $\mathfrak{u}(\mathbf{r})$ and $\mathfrak{u}(K, K)$ contain the common central element.

Since the variables $y_{i}^{\alpha}$ and $\bar{y}_{j}^{\alpha^{\prime}}$ are commuting, the tensors $C_{\alpha_{1} ; \ldots ; \alpha_{p} ; \alpha^{\prime}{ }_{1} ; \ldots ; \alpha_{q}^{\prime} q}^{i_{1} ; \ldots, i_{p} ; j_{1} ; \ldots ; j_{q}}(x)$ are symmetric with respect to permutation of any pair of upper and lower indices (for Greek indices of the same type). Hence every such a tensor can be decomposed into a direct sum of 
tensors described by irreducible representations of $\mathfrak{u}(\mathbf{r})$ as well as $\mathfrak{g l}_{K} \oplus \mathfrak{g l}_{K}$. The irreducible tensors have the symmetries described by a pair of YD $\mathbf{Y}\left(n_{1}, \ldots, n_{m}\right)$ and $\overline{\mathbf{Y}}\left(\bar{n}_{1}, \ldots, \bar{n}_{\bar{m}}\right)$ with $n_{1}+\ldots+n_{m}=p, \bar{n}_{1}+\ldots+\bar{n}_{\bar{m}}=q$.

Because of the symmetry with respect to exchange of pairs of indices, Young diagrams for color indices $i(j)$ and spinor-like indices $\alpha\left(\alpha^{\prime}\right)$ must have the same shape. Hence, primary fields in $\mathcal{M}_{M}^{M n k}$ are

$$
\begin{aligned}
& C_{\alpha^{1}\left(n_{1}\right), \ldots, \alpha^{m}\left(n_{m}\right) ; \quad \alpha^{\prime} 1\left(\bar{n}_{1}\right), \ldots, \alpha^{\prime} \bar{m}\left(\bar{n}_{\bar{m}}\right)}^{i^{1}\left(n_{1}\right), \ldots}, i^{m}(x)
\end{aligned}
$$

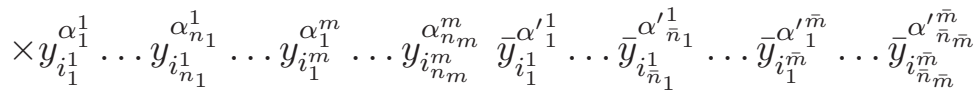

with tensors $C$ that obey the mutual tracelessness condition (4.5) and have definite symmetry properties in $y$ and $\bar{y}$ described by a pair of Young diagrams
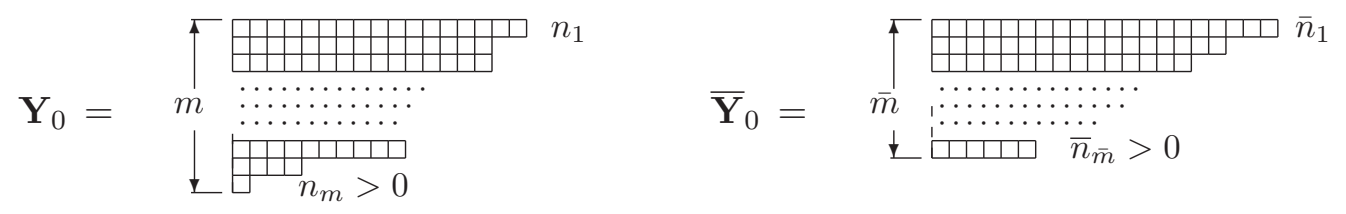

As a consequence of (4.5) and Lemma 1 on pg. 14 at $p=0$, the latter have to obey the condition

$$
\mathbf{r} \geq \bar{m}+m \geq 0 .
$$

In the Minkowski case, a pair of Young diagrams $\mathbf{Y}_{0}$ and $\overline{\mathbf{Y}}_{0}$ (4.7) will be called rank-r two-column dual to the pair $\mathbf{Y}_{1}(n_{1}, \ldots, n_{m}, \underbrace{1, \ldots, 1}_{q})$ and $\overline{\mathbf{Y}}_{1}(\bar{n}_{1}, \ldots, \bar{n}_{\bar{m}}, \underbrace{1, \ldots, 1}_{q})$

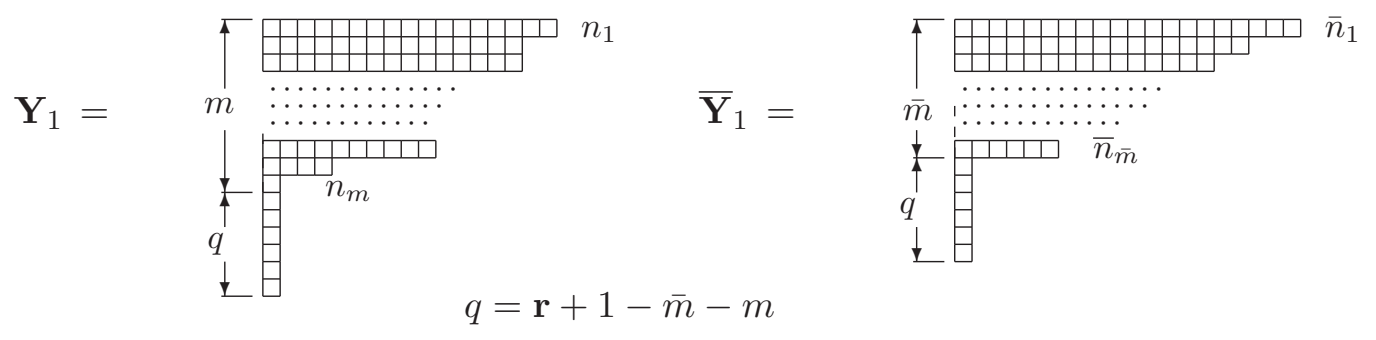

iff

$$
\begin{array}{ll}
\mathrm{h}_{1}\left(\mathbf{Y}_{0}\right)+\mathrm{h}_{1}\left(\overline{\mathbf{Y}}_{1}\right)=\mathrm{h}_{1}\left(\overline{\mathbf{Y}}_{0}\right)+\mathrm{h}_{1}\left(\mathbf{Y}_{1}\right)=\mathbf{r}+1, \quad \mathrm{~h}_{k}\left(\mathbf{Y}_{0}\right)=\mathrm{h}_{k}\left(\mathbf{Y}_{1}\right), \\
\\
\mathrm{h}_{k}\left(\overline{\mathbf{Y}}_{0}\right)=\mathrm{h}_{k}\left(\overline{\mathbf{Y}}_{1}\right) \quad \forall k \geq 2
\end{array}
$$

Equations of motion in $\mathcal{M}_{M}^{M n k}$ are most conveniently represented with the help of the projecting tensor

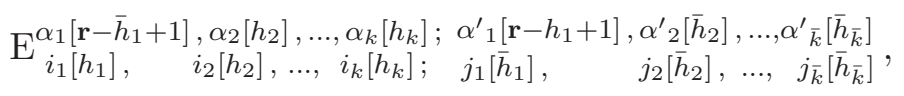


that obeys mutual tracelessness condition (4.5) and has symmetry described by the two pairs of mutually traceless Young diagrams

$$
\mathbf{Y}_{0}\left[h_{1}, h_{2}, h_{3}, \ldots, h_{k}\right], \quad \overline{\mathbf{Y}}_{0}\left[\bar{h}_{1}, \bar{h}_{2}, \ldots, \bar{h}_{\bar{k}}\right]
$$

in the color indices and their two-column dual

$$
\mathbf{Y}_{1}\left[\mathbf{r}-\bar{h}_{1}+1, h_{2}, \ldots, h_{k}\right], \quad \overline{\mathbf{Y}}_{1}\left[\mathbf{r}-h_{1}+1, \bar{h}_{2}, \ldots, \bar{h}_{\bar{k}}\right]
$$

in the spinor ones.

The following equation holds as a consequence of rank-r unfolded equations (1.8):

$$
\begin{aligned}
& \widehat{\mathrm{E}}_{\mathbf{Y}_{0}, \overline{\mathbf{Y}}_{0}} \mathcal{C}(y, \bar{y} \mid x)=0 \text {, } \\
& \widehat{\mathrm{E}}_{\mathbf{Y}_{0}, \overline{\mathbf{Y}}_{0}}:=\mathrm{E}^{\alpha_{1}\left[\mathbf{r}-\bar{h}_{1}+1\right], \alpha_{2}\left[h_{2}\right], \ldots, \alpha_{k}\left[h_{k}\right] ; \alpha^{\prime}{ }_{1}\left[\mathbf{r}-h_{1}+1\right], \alpha^{\prime}{ }_{2}\left[\bar{h}_{2}\right], \ldots, \alpha^{\prime} \bar{k}\left[\bar{h}_{\bar{k}}\right]}
\end{aligned}
$$

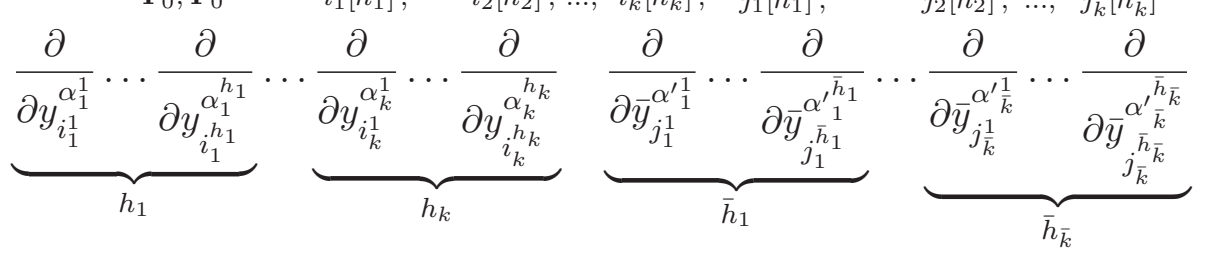

$$
\begin{aligned}
& \underbrace{\frac{\partial}{\partial x^{\alpha_{1}^{h_{1}+1}} \alpha^{h_{1}+1}} \cdots \frac{\partial}{\partial x_{1}^{\alpha_{1}^{\mathbf{r}+1-\bar{h}_{1}}} \alpha_{1}^{\alpha_{1}^{\mathbf{r}+1-h_{1}}}}}_{\mathbf{r}+1-h_{1}-\bar{h}_{1}} .
\end{aligned}
$$

Indeed, by virtue of (1.8), eq. (4.15) is equivalent to

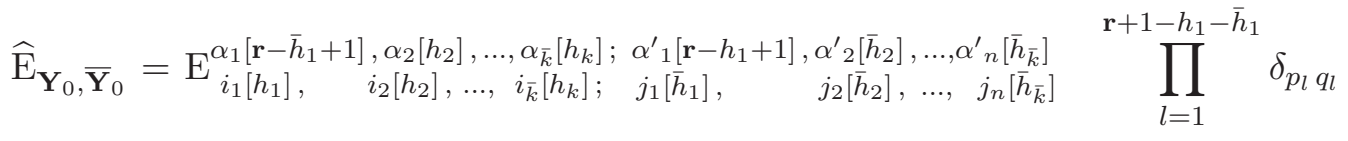

$$
\begin{aligned}
& \underbrace{\frac{\partial}{\partial y_{i_{1}^{1}}^{\alpha_{1}^{1}}} \cdots \frac{\partial}{\partial y_{i_{1}^{h_{1}}}^{\alpha_{1}}}}_{h_{1}} \cdots \underbrace{\frac{\partial}{\partial y_{i_{k}^{1}}^{\alpha_{k}^{1}}} \cdots \frac{\partial}{\partial y_{i_{k}^{h_{k}}}^{\alpha_{k}}}}_{h_{k}} \quad \underbrace{\frac{\partial}{\partial y_{p_{1}}^{\alpha_{1}+1}} \cdots \frac{\partial}{\partial y_{1}^{\alpha_{1}^{\mathbf{r}+1-h_{1}}}}}_{N=\mathbf{r}+1-h_{1}-\bar{h}_{1}}
\end{aligned}
$$

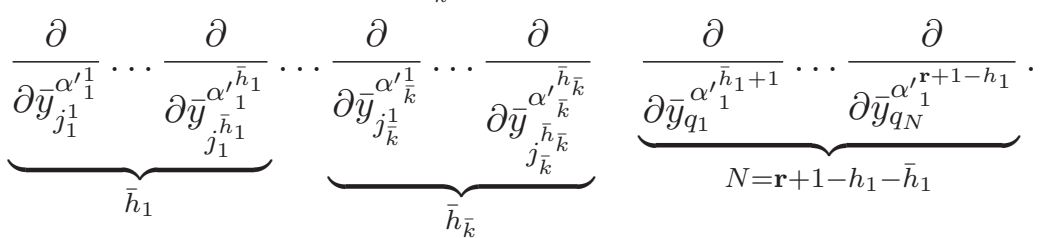

Since derivatives $\frac{\partial}{\partial Y_{j}^{\alpha^{\prime}}}$ commute while the indices in columns of YDs (4.12) and (4.13) are antisymmetrized, the indices $p_{1} \ldots p_{\mathbf{r}+1-h_{1}-\bar{h}_{1}}$ are antisymmetrized with the indices $i_{1}\left[h_{1}\right]$ while the indices $q_{1} \ldots q_{\mathbf{r}+1-h_{1}-\bar{h}_{1}}$ are antisymmetrized with the indices $j_{1}\left[\bar{h}_{1}\right]$. This yields zero by virtue of mutual tracelessness of tensor E (4.11) and Lemma 1 on pg. 14.

Since $\widehat{E}(4.15)$ is a homogeneous differential operator, eq. (4.14) is obeyed by primary fields (4.6). The nontrivial part of the analysis is to show that eq. (4.14) yields the full list of dynamical equations. This follows from the analysis of the cohomology groups $H^{0}\left(\sigma_{-}^{\mathbf{r}}{ }^{M n k}\right)$ and $H^{1}\left(\sigma_{-}^{\mathbf{r}}{ }^{M n k}\right)$ of $\sigma_{-}^{\mathbf{r}}{ }^{M n k}$ (1.8) for arbitrary $M=2 K$ outlined in section 5.2. 


\section{$4.24 d$ Minkowski space}

The dictionary between the tensor and two-component spinor notations is based on

$$
A^{\alpha \beta^{\prime}}=A^{a} \sigma_{a}^{\alpha \beta^{\prime}},
$$

where $\sigma_{a}^{\alpha \beta^{\prime}}(a=0,1,2,3)$ are four Hermitian $2 \times 2$ matrices. Let us list the $4 d$ Minkowski primary fields and their field equations for various $\mathbf{r}$.

As follows from the analysis of [9], the $\mathbf{r}=1$ primary fields are

$$
C(x), C(y \mid x), \bar{C}(\bar{y} \mid x) \text {. }
$$

These have symmetry properties described by the following pairs of Young diagrams

$$
\begin{array}{lll}
\mathbf{Y}_{0}=\bullet, & \overline{\mathbf{Y}}_{0}=\bullet ; \\
\mathbf{Y}_{0}=\bullet, & \overline{\mathbf{Y}}_{0}=\square \square ; \\
\mathbf{Y}_{0}=\square \square \square, & \overline{\mathbf{Y}}_{0}=\bullet .
\end{array}
$$

The consequences of (1.8) for $\mathbf{r}=1$

$$
\begin{aligned}
\varepsilon^{\alpha \beta} \varepsilon^{\alpha^{\prime} \beta^{\prime}} \frac{\partial^{2}}{\partial x^{\alpha \alpha^{\prime}} \partial x^{\beta \beta^{\prime}}} C(y, \bar{y} \mid x) & =0, \\
\varepsilon^{\alpha \beta} \frac{\partial^{2}}{\partial x^{\alpha \alpha^{\prime}} \partial y^{\beta}} C(y, \bar{y} \mid x)=0, & \varepsilon^{\alpha^{\prime} \beta^{\prime}} \frac{\partial^{2}}{\partial x^{\alpha \alpha^{\prime}} \partial \bar{y}^{\beta^{\prime}}} C(y, \bar{y} \mid x)=0
\end{aligned}
$$

impose the equations on primaries (4.18)

$$
\begin{aligned}
& \varepsilon^{\alpha \beta} \varepsilon^{\alpha^{\prime} \beta^{\prime}} \frac{\partial^{2}}{\partial x^{\alpha \alpha^{\prime}} \partial x^{\beta \beta^{\prime}}} C(x)=0, \\
& \varepsilon^{\alpha \beta} \frac{\partial}{\partial x^{\alpha \alpha^{\prime}} \partial y^{\beta}} C(y \mid x)=0, \quad \varepsilon^{\alpha^{\prime} \beta^{\prime}} \frac{\partial}{\partial x^{\alpha \alpha^{\prime}} \partial \bar{y}^{\beta^{\prime}}} \bar{C}(\bar{y} \mid x)=0 .
\end{aligned}
$$

The symmetry properties of the left-hand-sides of these equations are described by the following pairs of Young diagrams

$$
\begin{array}{lll}
\mathbf{Y}_{1}=\boxminus & , & \overline{\mathbf{Y}}_{1}=\boxminus ; \\
\mathbf{Y}_{1}=\square & , & \overline{\mathbf{Y}}_{1}=\boxminus \square ; \\
\mathbf{Y}_{1}=\boxminus \square & , & \overline{\mathbf{Y}}_{1}=\square .
\end{array}
$$

Consider the rank-two case. To obey (4.8), in the case of $M=4, \mathbf{r}=2$ primary fields (4.6) belong to the following list

$$
C(y \mid x), \quad C(\bar{y} \mid x), \quad C(y, \bar{y} \mid x)
$$


possessing symmetries described by pairs of Young diagrams (4.7)

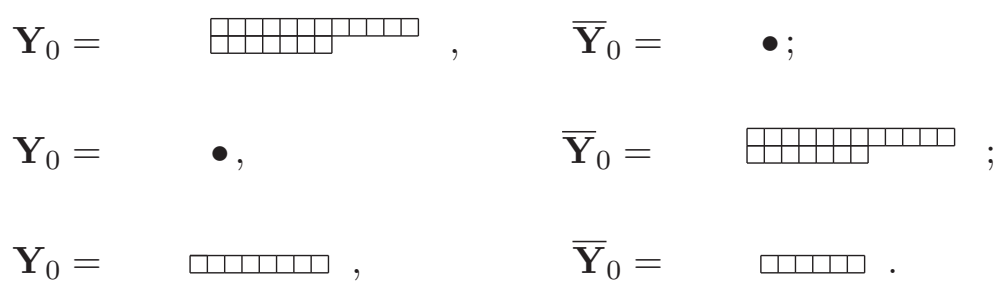

Since primary fields have to satisfy (1.8), to describe $C(y, \bar{y} \mid x)(4.22)$ it is convenient to use the language of highest-weight modules. Solutions to (1.8) form $\mathfrak{u}(\mathbf{r})$-modules, where the algebra $\mathfrak{u}(\mathbf{r})$ spanned by the generators

$$
\rho_{m}^{n}=y_{m}^{\alpha} \frac{\partial}{\partial y_{n}^{\alpha}}-\bar{y}^{\alpha^{\prime} n} \frac{\partial}{\partial \bar{y}^{\alpha^{\prime} m}}, \quad\left[\rho_{m}^{p}, \rho_{q}^{j}\right]=\delta_{q}^{p} \rho_{m}^{j}-\delta_{m}^{j} \rho_{q}^{p}
$$

commutes with $\sigma_{-}^{\mathbf{r} M n k}(4.2)$. Evidently, $\rho_{2}^{1} C\left(y_{1}, \bar{y}_{2} \mid x\right)=0$ and $\left(\rho_{1}^{2}\right)^{k} C\left(y_{1}, \bar{y}_{2} \mid x\right)$ with $0 \leq k \leq \max (n, \bar{n})$ form the full list of primary fields with symmetry properties (4.25). Note, that these results were used in [10] to describe free $4 d$ conformal primary currents.

Equations of motion are projected by tensors (4.11). Since $\alpha_{j}$ and $\alpha^{\prime}{ }_{j}$ take just two values, to be nonzero at $M=4, \mathbf{r}=2$, these should have $h_{1}=\bar{h}_{1}=1$ and $h_{j} \leq 1, \bar{h}_{j} \leq 1$ in YDs (4.12), (4.13). Hence, the primary fields associated with diagrams (4.23) and (4.24) are off-shell obeying no field equations, while those associated with diagrams (4.25) satisfy

$$
\begin{array}{r}
\varepsilon^{\alpha \beta} \varepsilon^{\alpha^{\prime} \beta^{\prime}} \frac{\partial^{3}}{\partial x^{\alpha \alpha^{\prime}} \partial y_{1}^{\beta} \partial \bar{y}_{2}^{\beta^{\prime}}} C(y, \bar{y} \mid x)=0, \quad \varepsilon^{\alpha \beta} \varepsilon^{\alpha^{\prime} \beta^{\prime}} \frac{\partial^{3}}{\partial x^{\alpha \alpha^{\prime}} \partial y_{2}^{\beta} \partial \bar{y}_{1}^{\beta^{\prime}}} C(y, \bar{y} \mid x)=0 \\
\varepsilon^{\alpha \beta} \varepsilon^{\alpha^{\prime} \beta^{\prime}} \frac{\partial}{\partial x^{\alpha \alpha^{\prime}}}\left(\frac{\partial^{2}}{\partial y_{1}^{\beta} \partial \bar{y}_{1}^{\beta^{\prime}}}-\frac{\partial^{2}}{\partial y_{2}^{\beta} \partial \bar{y}_{2}^{\beta^{\prime}}}\right) C(y, \bar{y} \mid x)=0 .
\end{array}
$$

These equations have symmetries of $\mathbf{Y}_{1}=\square$ and $\overline{\mathbf{Y}}_{1}=\square$.

For $\mathbf{r} \geq 3$, dynamical fields (4.6) are described by Young diagrams (4.7) with at most two rows. Since $H^{1}\left(\sigma_{-}^{\mathbf{r}}{ }^{M n k}\right)$ is empty for $\mathbf{r} \geq 3$, rank-r $\geq 3$ primary fields are off-shell.

\subsection{Higher $\sigma_{-}^{\text {r }}{ }^{n n k}$-cohomology in $\mathcal{M}_{M}^{M n k}$}

Due to anticommutativity of differentials, the differential forms $f\left(\xi^{\alpha \beta^{\prime}}\right)$ are described by pairs of mutually transposed Young diagrams $\mathbf{Y}$ and $\overline{\mathbf{Y}}$ related by the reflection with respect to the diagonal, i.e.,

$$
\mathrm{l}_{i}(\overline{\mathbf{Y}})=\mathrm{h}_{i}(\mathbf{Y}), \quad \mathrm{l}_{j}(\mathbf{Y})=\mathrm{h}_{j}(\overline{\mathbf{Y}})
$$

Such pairs of diagrams, whose role is analogous to that of almost symmetric $\operatorname{YDs} \mathbf{Y}_{A}(2.8)$ for the $\operatorname{Sp}(2 M)$ invariant sytems, will be denoted as $\mathbf{Y}_{a}, \overline{\mathbf{Y}}_{a}$, describing symmetry properties of $f\left(\xi^{\alpha \beta^{\prime}}\right)$ with respect to unprimed and primed indices, respectively. 
As sketched in section $5, H^{p}\left(\sigma_{-}^{\mathbf{r}}{ }^{M n k}\right)$ is characterized by a pair of mutually transposed Young diagrams $\mathbf{Y}_{a}$ and $\overline{\mathbf{Y}}_{a}$ such that $\left|\mathbf{Y}_{a}\right|=\left|\overline{\mathbf{Y}}_{a}\right|=p$ and a pair of Young diagrams $\mathbf{Y}_{0}$ and $\overline{\mathbf{Y}}_{0}$ obeying the condition $\mathrm{h}_{1}\left(\mathbf{Y}_{0}\right)+\mathrm{h}_{1}\left(\overline{\mathbf{Y}}_{0}\right) \leq \mathbf{r}$.

To describe cohomology groups we introduce the following infinite shift matrix $\mathcal{S}_{n m}\left(\mathbf{r} \mid \mathbf{Y}_{0}, \overline{\mathbf{Y}}_{0}\right)$

$$
\begin{array}{rlrl}
\mathcal{S}_{n m} & =\triangle_{m-n} & & \text { for } n<m, \quad n \geq 1, \\
\mathcal{S}_{n n} & =\triangle_{0}, & \\
\mathcal{S}_{m n}=\bar{\triangle}_{m-n} & \text { for } n<m,
\end{array}
$$

where

$$
\begin{array}{ll}
\triangle_{j} & =\mathrm{h}_{j}\left(\mathbf{Y}_{0}\right)-\mathrm{h}_{j+1}\left(\mathbf{Y}_{0}\right), \quad \bar{\triangle}_{j}=\mathrm{h}_{j}\left(\overline{\mathbf{Y}}_{0}\right)-\mathrm{h}_{j+1}\left(\overline{\mathbf{Y}}_{0}\right), \\
\triangle_{0} & =\mathbf{r}-\mathrm{h}_{1}\left(\mathbf{Y}_{0}\right)-\mathrm{h}_{1}\left(\overline{\mathbf{Y}}_{0}\right) .
\end{array}
$$

Pictorially

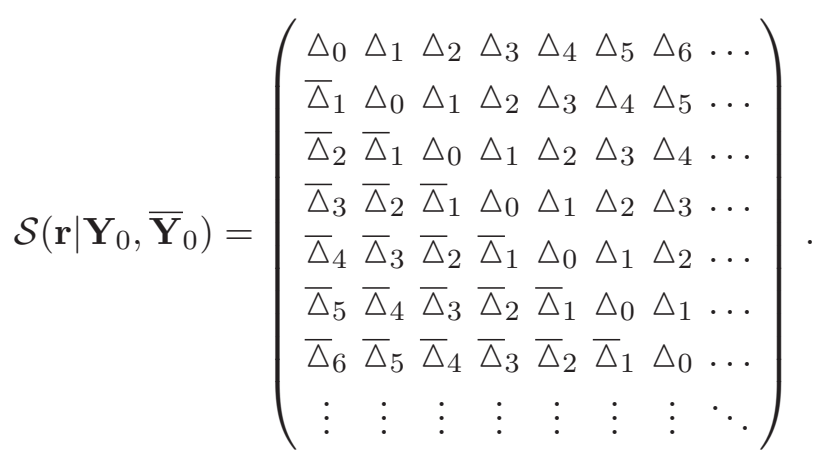

The cohomology group $H^{p}\left(\sigma_{-}^{\mathbf{r}}{ }^{M n k}\right)$ consists of the following pairs of $\mathfrak{g l}_{K} \oplus \mathfrak{g l}_{K}$ diagrams

$$
\begin{array}{ll}
\mathbf{Y}^{\prime}: & \mathrm{h}_{j}\left(\mathbf{Y}^{\prime}\right)=\mathrm{h}_{j}\left(\mathbf{Y}_{0}\right)+\mathrm{h}_{j}\left(\mathbf{Y}_{a}\right)+\sum_{i} \mathbb{S}_{i j}^{M s h}, \\
\overline{\mathbf{Y}^{\prime}}: & \mathrm{h}_{j}\left(\overline{\mathbf{Y}^{\prime}}\right)=\mathrm{h}_{j}\left(\overline{\mathbf{Y}}_{0}\right)+\mathrm{h}_{j}\left(\overline{\mathbf{Y}}_{a}\right)+\sum_{i} \mathbb{S}_{j i}^{M s h},
\end{array}
$$

where $\mathbb{S}_{i j}^{M s h}$ results from the intersection of the infinite shift matrix $\mathcal{S}\left(\mathbf{r} \mid \mathbf{Y}_{0}, \overline{\mathbf{Y}}_{0}\right)$ with $\mathbf{Y}_{a}$, i.e.,

$$
\mathbb{S}_{n m}^{M s h}=\theta\left(\mathrm{h}_{m}\left(\mathbf{Y}_{a}\right)-n\right) \mathcal{S}_{n m} .
$$

For example, for the Young diagram $\mathbf{Y}_{a}[6,6,5,5,4,2,2,1]$ the shift matrix is

$$
\mathbb{S}^{M s h}=\left(\begin{array}{llllllll}
\triangle_{0} & \Delta_{1} & \Delta_{2} & \Delta_{3} & \Delta_{4} & \Delta_{5} & \Delta_{6} & \Delta_{7} \\
\bar{\triangle}_{1} & \Delta_{0} & \Delta_{1} & \Delta_{2} & \Delta_{3} & \Delta_{4} & \Delta_{5} & 0 \\
\bar{\triangle}_{2} & \bar{\triangle}_{1} & \Delta_{0} & \Delta_{1} & \Delta_{2} & 0 & 0 & 0 \\
\bar{\triangle}_{3} & \bar{\triangle}_{2} & \bar{\triangle}_{1} & \Delta_{0} & \Delta_{1} & 0 & 0 & 0 \\
\bar{\triangle}_{4} & \bar{\triangle}_{3} & \bar{\Delta}_{2} & \bar{\Delta}_{1} & 0 & 0 & 0 & 0 \\
\bar{\triangle}_{5} & \bar{\triangle}_{4} & 0 & 0 & 0 & 0 & 0 & 0
\end{array}\right) .
$$




\section{$5 \quad \sigma_{-}$-cohomology analysis}

Our analysis generalizes those of [5], where the rank-two case was considered, and of [34], where conformal field equations in Minkowski space of any dimension were obtained. The main tool is the standard homotopy trick.

Let a linear operator $\Omega$ act in a linear space $V$ and satisfy $\Omega^{2}=0$. By definition, $H(\Omega)=\operatorname{ker} \Omega / \operatorname{Im} \Omega$ is the cohomology space. Let $\Omega^{*}$ be another nilpotent operator, $\left(\Omega^{*}\right)^{2}=0$. Then the operator

$$
\Delta=\left\{\Omega, \Omega^{*}\right\}
$$

satisfies $[\Omega, \Delta]=\left[\Omega^{*}, \Delta\right]=0$. From (5.1) it follows that $\Delta(\operatorname{ker} \Omega) \subset \operatorname{Im} \Omega$. Therefore $H(\Omega) \subset \operatorname{ker} \Omega / \Delta(\operatorname{ker} \Omega)$. Suppose now that $V$ is a Hilbert space in which $\Omega^{*}$ and $\Omega$ are conjugated. Then $\Delta$ is positive semi-definite. If the operator $\Delta$ is also quasifinitedimensional, i.e., $V=\sum \oplus V_{A}$ with finite-dimensional subspaces $V_{A}$ such that $\Delta\left(V_{A}\right) \subset V_{A}$ and $V_{A}$ is orthogonal to $V_{B}$ for $A \neq B$, then $\Delta$ can be diagonalized and it is easy to see that ker $\Omega / \Delta(\operatorname{ker} \Omega)=\operatorname{ker} \Delta \cap \operatorname{ker} \Omega$. Therefore, in this case,

$$
H(\Omega) \subset \operatorname{ker} \Delta \cap \operatorname{ker} \Omega \text {. }
$$

This formula is particularly useful for the practical analysis since, to calculate $H(\Omega)$, one can use the Homotopy equation

$$
\Delta F=0
$$

\subsection{Homotopy equation in $\mathcal{M}_{M}$}

Defining

$$
\frac{\partial}{\partial \xi^{B D}} \xi^{M N}=\frac{1}{2}\left(\delta_{B}^{M} \delta_{D}^{N}+\delta_{B}^{N} \delta_{D}^{M}\right)-\xi^{M N} \frac{\partial}{\partial \xi^{B D}},
$$

we observe that the operators

$$
\rho_{B}^{A}=2 \xi^{A D} \frac{\partial}{\partial \xi^{B D}}
$$

form $\mathfrak{g l}(M)$, obeying

$$
\begin{aligned}
{\left[\rho_{B}^{A}, \rho_{M}^{N}\right] } & =\delta_{B}^{N} \rho_{M}^{A}-\delta_{M}^{A} \rho_{N}^{B}, \\
{\left[\rho_{B}^{A}, \xi^{M N}\right] } & =\delta_{B}^{M} \xi^{A N}+\delta_{B}^{N} \xi^{A M} \\
\rho_{B}^{A} \rho_{A}^{B} & =(M+1) \rho_{A}^{A} .
\end{aligned}
$$

For

$$
\Omega^{\mathbf{r}}:=\sigma_{-}^{\mathbf{r}}=T_{A B} \xi^{A B}
$$

with $\sigma_{-}^{\mathbf{r}}$ (1.5) and $T_{A B}$ (3.18) we introduce

$$
\begin{aligned}
\Omega^{* \mathbf{r}} & =T^{C D} \frac{\partial}{\partial \xi^{C D}}, \quad\left(\Omega^{* \mathbf{r}}\right)^{2}=0, \\
\Delta^{\mathbf{r}} & =\left\{\Omega^{\mathbf{r}}, \Omega^{* \mathbf{r}}\right\} .
\end{aligned}
$$


An important property of $\Delta^{\mathbf{r}}(5.11)$ is that it is positive semi-definite because $\Omega^{\mathbf{r}}$ and $\Omega^{* \mathbf{r}}$ are conjugated in the positive-definite Fock space generated by $Y_{i}^{A}$ and $\xi^{A B}$ as creation operators.

By virtue of (3.19), (5.6) and (5.7), $\Delta^{\mathbf{r}}$ can be represented in the form

$$
\Delta^{\mathbf{r}}=\left(\mathcal{T}_{A}^{B}+\rho_{A}^{B}\right)\left(\mathcal{T}_{B}^{A}+\rho_{B}^{A}\right)+\left(T^{A B} T_{A B}-\mathcal{T}_{A}^{B} \mathcal{T}_{B}^{A}\right)+(\mathbf{r}-(M+1)) \rho_{A}^{A},
$$

where

$$
\mathcal{T}_{B}^{A}=: Y_{j}^{A} \frac{\partial}{\partial Y_{j}^{B}}=T_{B}^{A}-\frac{1}{2} \mathbf{r} \delta_{B}^{A}
$$

are generators of the $\mathfrak{g l}_{M}$ that act on $Y_{i}^{A}$.

By virtue of (3.17)-(3.21) an elementary computation yields

$$
T^{A B} T_{A B}-\mathcal{T}_{A}^{B} \mathcal{T}_{B}^{A}+(M+1-\mathbf{r}) \mathcal{T}_{A}^{A}=\frac{1}{2} \tau_{m n} \tau^{m n},
$$

where $\tau_{m n}$ are $\mathfrak{o}(\mathbf{r})$ generators $(3.20)$. Hence

$$
\Delta^{\mathbf{r}}=\nu_{B}^{A} \nu_{B}^{A}+\frac{1}{2} \tau_{m k} \tau^{m k}-(M+1-\mathbf{r}) \nu_{A}^{A},
$$

where

$$
\nu_{B}^{A}=\rho_{B}^{A}+\mathcal{T}_{B}^{A}
$$

are generators of the $\mathfrak{g}_{M}^{\text {tot }}$ that act on indices $A, B, \ldots$ carried by both $Y_{i}^{A}$ and $\xi^{A B}$. The first and second terms on the r.h.s. of (5.15) are the quadratic Casimir operators of the algebras $\mathfrak{g}_{M}^{\text {tot }}$ and $\mathfrak{o}(\mathbf{r})$, respectively.

The computation in terms of fermionic oscillator realization of generators of the algebra $\mathfrak{g l}_{M}$, that makes the antisymmetrization manifest, yields (see e.g., [34])

$$
\nu_{B}^{A} \nu_{B}^{A}=-\sum_{i} H_{i}\left(H_{i}-M-1-2(i-1)\right)
$$

where $i$ enumerates height- $H_{i}$ columns of the $\mathfrak{g l}_{M}$ YD $\mathbf{Y}^{\prime}\left[H_{1}, H_{2}, \ldots\right]$.

As mentioned above, $\mathbf{Y}_{0}$ can be treated both as a $\mathfrak{g l}(M) \mathrm{YD}$, and as a $\mathfrak{o}(\mathbf{r}) \mathrm{YD}$. Recall that, in addition to the indices of a $\mathfrak{o}(\mathbf{r})$-traceless $\mathrm{YD} \mathbf{Y}_{0}$, the diagram $\mathbf{Y}^{\prime}$ treated as an $\mathfrak{o}(\mathbf{r})$ YD can contain indices carried by products of $\delta^{i j}$. Then the respective $\mathfrak{g l}(M)$ tensor contains products of the "tracefull" combinations (3.41) that, being symmetric in the indices $A, B$, are described by $\mathbf{Y}[1,1]$. In addition, it can contain indices carried by the differentials $\xi^{A B}$ also described by $\mathbf{Y}[1,1]$.

As a result

$$
\mathbf{Y}^{\prime}\left[H_{1}, H_{2}, \ldots\right] \in \mathbf{Y}_{0}\left[h_{1}, h_{2}, \ldots\right] \otimes \underbrace{\mathbf{Y}[1,1] \otimes \ldots \otimes \mathbf{Y}[1,1]}_{N}
$$

for some $N$. Here the pairs of symmetric indices carried by $U^{A B}(3.41)$ and $\xi^{A B}$ are symmetrized and antisymmetrized, respectively. 
Analogous computation for the orthogonal algebra yields

$$
\tau_{m k} \tau^{m k}=2 \sum_{j} h_{i}\left(h_{i}-\mathbf{r}-2(i-1)\right)
$$

where $i$ enumerates height- $h_{i}$ columns of the $\mathfrak{o}(\mathbf{r})$-traceless $\mathrm{YD} \mathrm{Y}_{0}\left[h_{1}, h_{2}, \ldots\right]$.

Hence, eq. (5.12) yields

$$
\Delta^{\mathbf{r}}=-\sum_{i} H_{i}\left(H_{i}-\mathbf{r}-2(i-1)\right)+\sum_{j} h_{j}\left(h_{j}-\mathbf{r}-2(j-1)\right) .
$$

By virtue of eqs. (2.4) this yields

$$
\Delta^{\mathbf{r}}=2 \chi^{\frac{1}{2}(\mathbf{r}-1)}\left(\mathbf{Y}^{\prime}\right)-2 \chi^{\frac{1}{2}(\mathbf{r}-1)}\left(\mathbf{Y}_{0}\right) .
$$

Since $\chi^{a}$ is additive, from (5.21) it follows that

$$
\chi^{\frac{1}{2}(\mathbf{r}-1)}\left(\mathbf{Y}^{\prime}\right)-\chi^{\frac{1}{2}(\mathbf{r}-1)}\left(\mathbf{Y}_{0}\right) \equiv \chi^{\frac{1}{2}(\mathbf{r}-1)}\left(\mathbf{Y}^{\prime} \backslash \mathbf{Y}_{0}\right)
$$

where summation in $\chi^{\frac{1}{2}(\mathbf{r}-1)}\left(\mathbf{Y}^{\prime} \backslash \mathbf{Y}_{0}\right)$ is over the cells $S(x, y)$ of the supplement of $\mathbf{Y}_{0}$ in $\mathbf{Y}^{\prime}$ with the convention that $\mathbf{Y}_{0}$ is situated in the upper left corner of $\mathbf{Y}^{\prime}$.

Consider Homotopy equation $\Delta^{\mathbf{r}} F=0$ (5.3) on a tensor $F$ obeying symmetry properties of $\mathbf{Y}^{\prime}\left[H_{1}, H_{2}, \ldots\right]$ (5.18). By virtue of eqs. (2.4), (5.20) it takes the form

$$
\chi^{\frac{1}{2}(\mathbf{r}-1)}\left(\mathbf{Y}^{\prime} \backslash \mathbf{Y}_{0}\right)=0
$$

Note that since equation (5.23) is independent of $M$, the assumption of section 3.2 that $M$ is large enough to avoid the restriction on the maximal height of $\mathfrak{g l}_{M^{-}}$Young diagrams does not restrict the generality.

Analogously to [34], to find the complete set of solutions to the homotopy equation one can use the positive semi-definiteness of $\Delta^{\mathrm{r}}$. Indeed, nontrivial cohomology can appear at $\Delta^{\mathbf{r}}=0$ which is its minimal possible value. Equation (2.3) implies that for a given set of cells of a $\mathfrak{g l}(M)$-diagram, the minimal value of $\chi^{\frac{1}{2}(\mathbf{r}-1)}\left(\mathbf{Y}^{\prime} \backslash \mathbf{Y}_{0}\right)$ is reached when all cells are situated maximally South-West, i.e., possible values of $i$ and $j$ in (2.3) are maximized and minimized, respectively. This minimization will be referred to as South-West (SW) principle.

For a given traceless $\mathrm{YD} \mathbf{Y}_{0}$ the minimization occurs with respect to the cells associated with the differentials $\xi^{A B}$ forming an almost symmetric $\mathrm{YD} \mathbf{Y}_{A}(2.8)$ and the tracefull blocks $U^{A B}$ (3.41) forming a $\mathrm{YD} \mathbf{Y}_{\delta}(2.7)$. Any solution $\mathbf{Y}^{\prime}$ of (5.23) is given by

$$
\mathbf{Y}^{\prime} \in S W\left(\mathbf{Y}_{0} \otimes \mathbf{Y}_{\delta} \otimes \mathbf{Y}_{A}\right)
$$

for an appropriate $\mathbf{Y}_{\delta}$, where $S W\left(\mathbf{Y}_{1} \otimes \mathbf{Y}_{2} \otimes \ldots\right)$ denotes maximally South-West components $\left(\sim S W\right.$-diagrams) of the tensor product $\mathbf{Y}_{1} \otimes \mathbf{Y}_{2} \otimes \ldots$ For given $\mathbf{Y}_{0}$ and $\mathbf{Y}_{A}$, the problem is to find $\mathbf{Y}_{\delta}(2.7)$ such that the maximally South-West component of the tensor product $\mathbf{Y}_{0} \otimes \mathbf{Y}_{\delta} \otimes \mathbf{Y}_{A}$ solves (5.23). 
The details of the analysis of the Homotopy equation are given in appendix A. However, for the final results presented in section 3.2 it is easy to check that $\mathbf{Y}^{\prime}$ (3.49) solves homotopy equation (5.23). Indeed, substitution of $\mathbf{Y}^{\prime}$ (3.49) into (5.23) yields using (2.4)

$$
\chi^{\frac{1}{2}(\mathbf{r}-1)}\left(\mathbf{Y}^{\prime} \backslash \mathbf{Y}_{0}\right)=\sum_{j \leq \mathbf{n}} \sum_{k=1}^{\mathfrak{h}_{j}}\left(\mathbb{S}_{k j}^{s h}+1\right)\left(j-h_{j}-\sum_{n<k}\left(\mathbb{S}_{n j}^{s h}+1\right)-\frac{1}{2}\left(\mathbb{S}_{k j}^{s h}+2\right)+\frac{1}{2}(\mathbf{r}-1)\right) .
$$

For $\mathbf{Y}_{A}=\mathbb{Y}^{\text {nest }}\left\{a_{1}, \ldots a_{\mathbf{n}}\right\}$ (2.10), using the nested structure of the nested shift matrix (3.48) along with eq. (3.46), the l.h.s. of (5.25) can be rewritten in the form

$$
\chi^{\frac{1}{2}(\mathbf{r}-1)}\left(\mathbf{Y}^{\prime} \backslash \mathbf{Y}_{0}\right)=\sum_{k \leq \mathbf{n}} \sum_{m=k}^{a_{k}}\left(\mathbb{S}_{k m}^{s h}+1\right)\left\{A_{m k}+B_{m k}\right\}
$$

where

$$
\begin{aligned}
& A_{m k}=\mathbf{r}-h_{k}-h_{m+1}-\mathbb{S}_{m k}^{s h}-\sum_{n<m} \mathbb{S}_{n k}^{s h}-\sum_{n<k} \mathbb{S}_{m n}^{s h}, \\
& B_{m k}=m+k-1-1-\sum_{n<m} 1-\sum_{n<k} 1 .
\end{aligned}
$$

One can see that $A_{m k}=0$ for all $k, m$ by virtue of (3.47) while $B_{m k}$ also vanishes.

\subsection{Sketch of the $\sigma_{-}$-cohomology analysis in $\mathcal{M}_{M}^{M n k}$}

Here we consider $\sigma_{-}$-cohomology in the generalised Minkowski space $\mathcal{M}_{M}^{M n k}$ with $M=2 K$, i.e., $H\left(\sigma_{-}^{\mathbf{r} M n k}\right)$ for $\sigma_{-}^{\mathbf{r} M n k}(4.2)$.

The operators

$$
\phi_{\beta}^{\alpha}=\xi^{\alpha \gamma^{\prime}} \frac{\partial}{\partial \xi^{\beta \gamma^{\prime}}}, \quad \bar{\phi}_{\beta^{\prime}}^{\alpha^{\prime}}=\xi^{\alpha \alpha^{\prime}} \frac{\partial}{\partial \xi^{\alpha \beta^{\prime}}},
$$

form $\mathfrak{g l}_{K}(\mathbb{C})$ while the operators

$$
T^{\alpha \beta^{\prime}}=y_{j}^{\alpha} y^{\beta^{\prime} j}, \quad T_{\alpha \beta^{\prime}}=\frac{\partial}{\partial y_{j}^{\alpha}} \frac{\partial}{\partial y^{\beta^{\prime} j}}, \quad T_{\beta}^{\alpha}=\frac{1}{2}\left\{y_{j}^{\alpha}, \frac{\partial}{\partial y_{j}^{\beta}}\right\}, \quad T_{\beta^{\prime}}^{\alpha^{\prime}}=\frac{1}{2}\left\{\bar{y}_{j}^{\alpha^{\prime}}, \frac{\partial}{\partial \bar{y}_{j}^{\beta^{\prime}}}\right\}
$$

form $\mathfrak{u}(K, K)$.

Setting

$$
\Omega_{M n k}^{\mathbf{r}}=\sigma_{-}^{\mathbf{r} M n k}=T_{\alpha \beta^{\prime}} \xi^{\alpha \beta^{\prime}}, \quad \Omega_{M n k}^{* \mathbf{r}}=T^{\alpha \beta^{\prime}} \frac{\partial}{\partial \xi^{\alpha \beta^{\prime}}},
$$

we obtain using (5.30), (4.26)

$$
\Delta_{M n k}^{\mathbf{r}}=\frac{1}{2}\left(\nu_{\beta}^{\alpha} \nu_{\alpha}^{\beta}+\bar{\nu}_{\beta^{\prime}}^{\alpha^{\prime}} \bar{\nu}_{\alpha^{\prime}}^{\beta^{\prime}}-\rho_{m}^{p} \rho_{p}^{m}+(\mathbf{r}-K)\left(\nu_{\alpha}^{\alpha}+\bar{\nu}_{\alpha^{\prime}}^{\alpha^{\prime}}\right)\right),
$$

where

$$
\nu_{\beta}^{\alpha}=\phi_{\beta}^{\alpha}+\mathcal{T}_{\beta}^{\alpha}, \quad \bar{\nu}_{\beta^{\prime}}^{\alpha^{\prime}}=\phi_{\beta^{\prime}}^{\alpha^{\prime}}+\mathcal{T}_{\beta^{\prime}}^{\alpha^{\prime}}, \quad \mathcal{T}_{\beta}^{\alpha}=y_{j}^{\alpha} \frac{\partial}{\partial y_{j}^{\beta}}, \quad \mathcal{T}_{\beta^{\prime}}^{\alpha^{\prime}}=\bar{y}_{j}^{\alpha^{\prime}} \frac{\partial}{\partial \bar{y}_{j}^{\beta^{\prime}}}
$$


are $\mathfrak{g l}_{K}(\mathbb{C})$ generators while

$$
\rho_{m}^{n}=y_{m}^{\alpha} \frac{\partial}{\partial y_{n}^{\alpha}}-\bar{y}^{\alpha^{\prime} n} \frac{\partial}{\partial \bar{y}^{\alpha^{\prime} m}}
$$

are $\mathfrak{u}(\mathbf{r})$-generators (4.26). The sum of the first two terms and the third one on the r.h.s. of (5.32) are the quadratic Casimir operators of $\mathfrak{g l}_{K}(\mathbb{C})$ and $\mathfrak{u}(\mathbf{r})$, respectively.

The $\mathfrak{u}(\mathbf{r})$-generators $\rho_{m}^{n}$ (4.26) commute with $\mathfrak{u}(K, K)$ (5.30). Hence $\mathfrak{u}(\mathbf{r})$ acts on solutions of the higher-rank equations which form $\mathfrak{u}(\mathbf{r})$-modules. Irreducible $\mathfrak{u}(\mathbf{r})$-modules satisfy the mutual tracelessness conditions (4.4) equivalent to

$$
T_{\alpha \beta^{\prime}} P(y, \bar{y}, \xi \mid x)=0 .
$$

An elementary computation in terms of fermionic oscillator realization of $\mathfrak{u}(\mathbf{r})$, that makes the anti-symmetrization manifest, yields with the help of mutual tracelessness condition (5.33)

$$
\rho_{m}^{p} \rho_{p}^{m}=-\sum_{i} h_{i}\left(h_{i}-\mathbf{r}-2(i-1)-1\right)-\sum_{j} \bar{h}_{j}\left(\bar{h}_{j}-\mathbf{r}-2(j-1)-1\right),
$$

where $h_{i}$ and $\bar{h}_{i}$ are heights of the respective columns of $\mathbf{Y}_{0}\left[h_{1} \ldots, h_{0}\right]$ and $\overline{\mathbf{Y}}_{0}\left[\bar{h}_{1}, \ldots, \bar{h}_{k}\right]$. Using (5.17) for the Casimir operators $\nu_{\beta}^{\alpha} \nu_{\alpha}^{\beta}$ and $\bar{\nu}_{\beta^{\prime}}^{\alpha^{\prime}} \bar{\nu}_{\alpha^{\prime}}^{\beta^{\prime}}$, eq. (5.32) yields

$$
\begin{aligned}
\Delta_{M n k}^{\mathrm{r}}= & \frac{1}{2}\left(-\sum_{m} H_{m}\left(H_{m}-2(m-1)\right)-\sum_{n} \bar{H}_{n}\left(\bar{H}_{n}-2(n-1)\right)\right. \\
& \left.+\sum_{i} h_{i}\left(h_{i}-2(i-1)\right)+\sum_{j} \bar{h}_{j}\left(\bar{h}_{j}-2(j-1)\right)+(1+\mathbf{r}) \sum_{p}\left(H_{p}+\bar{H}_{p}-h_{p}-\bar{h}_{p}\right)\right),
\end{aligned}
$$

where $m$ enumerates columns of the $\mathfrak{g l}_{K}(\mathbb{C}) \mathrm{YD} \mathrm{Y}^{\prime}\left[H_{1}, H_{2} \ldots\right]$ and $n$ enumerates columns of the $\mathfrak{g l}_{K}(\mathbb{C})$ YD $\overline{\mathbf{Y}}^{\prime}\left[\bar{H}_{1}, \bar{H}_{2} \ldots\right]$.

Analogously to section 5.1, in addition to indices associated with the given mutually traceless $\mathbf{Y}_{0}$ and $\overline{\mathbf{Y}}_{0}$, the diagrams $\mathbf{Y}^{\prime}$ and $\overline{\mathbf{Y}}^{\prime}$ contain indices of the differentials $\xi^{\alpha \beta^{\prime}}$ and of the $\mathfrak{u}(\mathbf{r})$ invariant tracefull combinations

$$
u^{\alpha \beta^{\prime}}=\delta^{i j} y_{i}^{\alpha} \bar{y}_{j}^{\beta^{\prime}} .
$$

As a result $\mathbf{Y}^{\prime} \otimes \overline{\mathbf{Y}}^{\prime}$ belongs to $\mathbf{Y}_{0} \otimes \overline{\mathbf{Y}}_{0} \underbrace{\otimes \mathbf{Y}[1] \ldots \otimes \mathbf{Y}[1]}_{N} \underbrace{\otimes \overline{\mathbf{Y}}[1] \ldots \otimes \overline{\mathbf{Y}}[1]}_{N}$ for some $N \geq 1$. Hence $H_{i}=h_{i}+s_{i}, \bar{H}_{i}=\bar{h}_{i}+\bar{s}_{i}$ with some $s_{i} \geq 0, \bar{s}_{i} \geq 0, \sum s_{i}=\sum \bar{s}_{i}=N$.

By virtue of eqs. (5.35) and (2.4) the equation $\Delta_{M n k}^{\mathrm{r}}=0$ yields

$$
\chi^{\frac{1}{2} \mathbf{r}}\left(\mathbf{Y}^{\prime} \backslash \mathbf{Y}_{0}\right)+\chi^{\frac{1}{2} \mathbf{r}}\left(\overline{\mathbf{Y}}^{\prime} \backslash \overline{\mathbf{Y}}_{0}\right)=0,
$$

where, as before, summation in $\chi^{\frac{1}{2} \mathbf{r}}\left(\mathbf{Y}^{\prime} \backslash \mathbf{Y}_{0}\right)$ is over the cells $S(x, y)$ of the supplement of $\mathbf{Y}_{0}$ in $\mathbf{Y}^{\prime}$ with the convention, that $\mathbf{Y}_{0}$ is situated in the upper left corner of $\mathbf{Y}^{\prime}$, and analogously for the conjugated YDs.

This form of the homotopy equation allows us to make sure that any pair of Young diagrams $\mathbf{Y}^{\prime}, \overline{\mathbf{Y}}^{\prime}$ of the form (4.32) indeed solves (5.37), therefore describing the $\sigma^{M n k_{-}}$ cohomology. Using the positive semi-definiteness of $\Delta_{M n k}^{\mathbf{r}}$ and the South-West principle along the lines of section 5.1 and appendix A, it can be shown that the list of solutions of section 4.3 is complete. 


\section{Conclusion}

Results of this paper raise a number of interesting problems. One of the most interesting is to study conserved charges generated by the constructed currents. A single conserved current is expected to generate many different charges upon integration with different global symmetry parameters $\eta$. (For example, a traceless stress tensor generates the full conformal algebra being integrated with the parameters of translations, Lorentz rotations, dilatations and special conformal transformations.) See, e.g., [10] for the analysis of this issue for rank-two conserved currents. It is therefore important to find the full space of the corresponding symmetry parameters $\eta$ leading to independent charges. An interesting peculiarity of this analysis is that, as shown in [35] for the case of rank two, to obtain nonzero charges in Minkowski subspace of $\mathcal{M}_{M}$ it is necessary to consider parameters $\eta$ that are singular in some of coordinates in $\mathcal{M}_{M}$ transversal to Minkowski space. It is therefore necessary to find what is an appropriate singularity of $\eta$ in the general case of any rank that gives rise to non-zero conserved charges.

Another peculiarity is that, being multilinear in the dynamical fields, the charges resulting from the proposed currents cannot be represented as integrals in usual Minkowski space, requiring integration over a larger space like $\mathcal{M}_{M}$ or its product with the twistor space. Nevertheless, being nonlocal from the perspective of Minkowski space, the charges are well-defined and should form some algebra. An interesting question is what is this algebra and, specifically, what is its relation to the multiparticle algebra proposed recently in [36] and [10] where it was shown, in particular, that the usual bilinear (1.e., rank-two) currents give rise to a set of charges that forms the higher-spin algebra.

A very interesting possible application of the obtained results can be related to the analysis of multi-particle amplitudes in QFT. Indeed, the higher-rank fields considered in this paper can be interpreted as being associated with the asymptotic states in the scattering processes which are on shell, i.e., obey free field equations. The idea is to associate the constructed conserved charges with the amplitudes. Of course, this will not allow us to determine amplitudes exactly since for this it is necessary to determine the parameters $\eta$ in (3.65) which remain arbitrary functions of the respective twistor variables in our approach and have to be determined by the dynamics of a nonlinear model in question. However, this will determine the amplitude kinematics.

The reason why we believe that the amplitudes should be associated with the higherrank conserved charges is that, being represented by integrals of on-shell closed forms, in this case they will only be determined by certain singularities independent of local variations of the integration contour. This interpretation is not only very similar to the what happens in the Grassmanian computations (see e.g. [37, 38] and references therein) but can open a unique opportunity for establishing explicit relation between the space-time computational schemes and those in the twistor space. To this end the constructed conserved charges should be extended to differential forms in the correspondence space unifying space-time with the twistor space. This program was initiated in [35] for general tensor products. We anticipate that this construction allows an extension to irreducible conserved charges considered in this paper, integrated over the full correspondence space with appropriate 
singular parameters analogously to the construction of Minkowski currents from those in $\mathcal{M}_{4}$ presented in [35]. We leave this exciting problem for the future.

Finally, the analysis of higher cohomology performed in this paper may have applications to the construction of equations of motion of higher gauge theories associated with higher-rank fields. The latter are related to multiparticle states in the original field theoretic model. Such higher gauge theories are likely to be related to string-like higher-spin gauge theories.

\section{Acknowledgments}

We are grateful to Nathan Berkovits for hospitality at ICTP-SAIFR Sao-Paulo where a part of this work was done. Also we thank the organizers and participants of winter 2014 Kavli Institute for Theoretical Physics in Santa Barbara program "New Methods in Nonperturbative Quantum Field Theory", where this work was continued, for creation of friendly and productive atmosphere and stimulating conversations. The authors are grateful to Leonid Bork for the stimulating discussion and Yegor Goncharov for pointing out a typo in the draft. The first version of this work was supported in part by RFBR Grant No 11-02-00814-a. The extension of the original version by the evaluation of higher $\sigma_{-}^{\mathbf{r}}$ - cohomologies in $\mathcal{M}_{M}$ and $\mathcal{M}_{M}^{M n k}$ presented in sections 3.2 and 4.3 and in appendix A was supported by the Russian Science Foundation grant 14-42-00047.

\section{A Details of analysis of homotopy equation in $\mathcal{M}_{M}$}

For any pair of Young diagrams $\mathbf{Y}_{1,2}, \mathbf{Y}_{1} \underset{\mathrm{SW}}{\otimes} \mathbf{Y}_{2}=\mathbf{Y}_{2} \underset{\mathrm{SW}}{\otimes} \mathbf{Y}_{1}$ is defined as

$$
\mathbf{Y}_{1} \underset{\mathrm{SW}}{\otimes} \mathbf{Y}_{2}: \quad \mathrm{h}_{j}\left(\mathbf{Y}_{1} \underset{\mathrm{SW}}{\otimes} \mathbf{Y}_{2}\right)=\mathrm{h}_{j}\left(\mathbf{Y}_{1}\right)+\mathrm{h}_{j}\left(\mathbf{Y}_{2}\right) \quad \forall j
$$

Evidently, $\mathbf{Y}_{1} \underset{\mathrm{SW}}{\otimes} \mathbf{Y}_{2}$ is a Young diagram. From definition (2.2) it follows that

$$
\chi^{a}\left(\mathbf{Y}_{1} \underset{\mathrm{SW}}{\otimes} \mathbf{Y}_{2}\right)=\chi^{a}\left(\mathbf{Y}_{1}\right)+\chi^{a}\left(\mathbf{Y}_{2}\right)-\sum_{j} h_{j}\left(\mathbf{Y}_{1}\right) h_{j}\left(\mathbf{Y}_{2}\right) .
$$

To solve homotopy equation (5.23) we observe that at sufficiently large $M$ a solution $\mathbf{Y}^{\prime}$ (5.24) to (5.23) has the form

with some

$$
\mathbf{Y}^{\prime}=\mathbf{Y}^{\prime \prime} \underset{\mathrm{SW}}{\otimes} \mathbf{Y}_{A}
$$

$$
\mathbf{Y}^{\prime \prime} \equiv \mathbf{Y}^{\prime \prime}\left\{\mathbf{Y}_{0}, \mathbf{Y}_{\delta}\right\} \in \mathbf{Y}_{0} \otimes \mathbf{Y}_{\delta}
$$

Indeed, since there are no constraints on the heights of components of $\mathbf{Y}_{1} \otimes \mathbf{Y}_{2}$ at sufficiently large $M$ for given $\mathfrak{g l}_{M}$-diagrams $\mathbf{Y}_{1}$ and $\mathbf{Y}_{2}, \mathbf{Y}_{1} \underset{\mathrm{SW}}{\otimes} \mathbf{Y}_{2}$ is nonzero and maximally SouthWest. Note that a similar argument cannot be applied to the construction of $\mathbf{Y}^{\prime \prime}$ since $\mathbf{Y}_{0} \otimes \mathbf{Y}_{\delta}$ can be zero by Lemma 1 (see pg. 14).

Hence, eq. (5.23) takes the form

$$
\Delta^{\mathbf{r}}=2 \chi^{\frac{1}{2}(\mathbf{r}-1)}\left(\left(\mathbf{Y}^{\prime \prime} \underset{\mathrm{SW}}{\otimes} \mathbf{Y}_{A}\right) / \mathbf{Y}_{0}\right)=0
$$


From (A.1) and (A.2) it follows that for any YD $\mathbf{Y}$ and almost symmetric YD $\mathbf{Y}_{A}$

$$
\chi^{\frac{1}{2}(\mathbf{r}-1)}\left(\mathbf{Y} \underset{\mathrm{SW}}{\otimes} \mathbf{Y}_{A}\right)=\chi^{\frac{1}{2}(\mathbf{r}-1)}(\mathbf{Y})+\frac{1}{2} \sum_{i=1}^{\mathrm{l}_{1}\left(\mathbf{Y}_{A}\right)} \mathrm{h}_{i}\left(\mathbf{Y}_{A}\right)\left(\mathbf{r}-2 \mathrm{~h}_{i}(\mathbf{Y})\right) .
$$

As a result, using additivity of $\chi^{a}(2.3)$, condition (A.5) amounts to

$$
\chi^{\frac{1}{2}(\mathbf{r}-1)}\left(\mathbf{Y}^{\prime \prime}\right)-\chi^{\frac{1}{2}(\mathbf{r}-1)}\left(\mathbf{Y}_{0}\right)+\frac{1}{2} \sum_{i}^{\mathfrak{l}_{1}\left(\mathbf{Y}_{A}\right)} \mathfrak{h}_{i}\left(\mathbf{r}-2 \mathcal{H}_{i}\right)=0,
$$

where

$$
\mathfrak{h}_{i}=\mathrm{h}_{i}\left(\mathbf{Y}_{A}\right), \quad \mathcal{H}_{i}=\mathrm{h}_{i}\left(\mathbf{Y}^{\prime \prime}\right)
$$

Since $\Delta^{\mathbf{r}}$ is positive semi-definite, eq. (A.6) implies that, for given $\mathbf{Y}_{0}$ and $\mathbf{Y}^{\prime \prime}$, a solution to (A.7) demands $\mathbf{Y}_{A}$ to minimize the last term, i.e., if $\mathbf{Y}_{A}$ solves (A.7) then

$$
\sum_{j}^{\mathrm{l}_{1}\left(\widetilde{\mathbf{Y}}_{A}\right)} \mathrm{h}_{j}\left(\widetilde{\mathbf{Y}}_{A}\right)\left(\mathbf{r}-2 \mathcal{H}_{j}\right) \geq \sum_{i}^{\mathrm{l}_{1}\left(\mathbf{Y}_{A}\right)} \mathrm{h}_{i}\left(\mathbf{Y}_{A}\right)\left(\mathbf{r}-2 \mathcal{H}_{i}\right)
$$

for any almost symmetric $\widetilde{\mathbf{Y}}_{A}$. Consideration of almost symmetric diagrams $\tilde{\mathbf{Y}}_{A}$ resulting from varying $\mathbf{Y}_{A}$ by a pair of cells severely restricts $\mathcal{H}_{i}$ allowed by (A.9). Analogous trick applied to the Kronecker YD $\mathbf{Y}_{\delta}$ makes it possible to solve (A.7).

For instance, consider $\mathbb{Y}^{\text {nest }}\{\underbrace{10,9,8}_{3}, \underbrace{4,3}_{2}\}$ that consists of two block hooks.
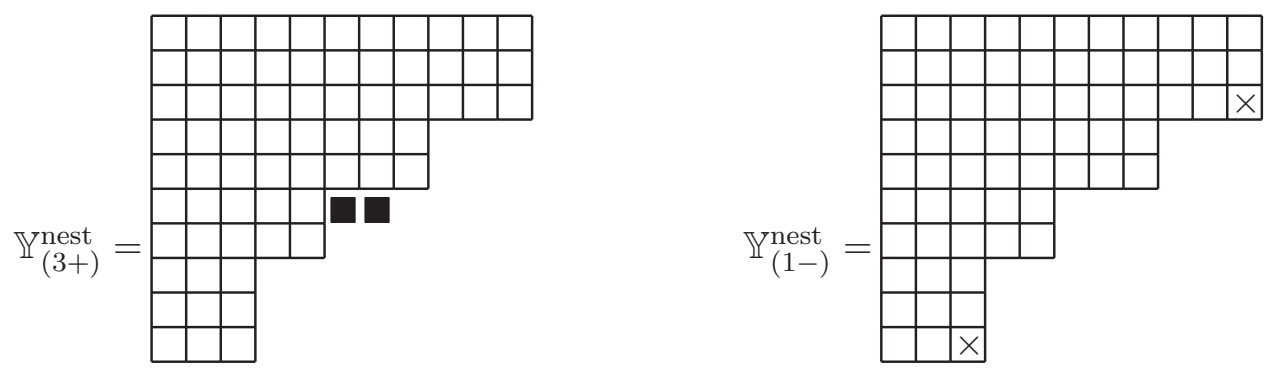

(A.10)

The black cells of the left YD exemplify an addition of a pair to form the third block hook, while the crossed cells of the right YD can be removed from the first block hook to produce another almost symmetric diagram.

Another useful example is a rectangular block.
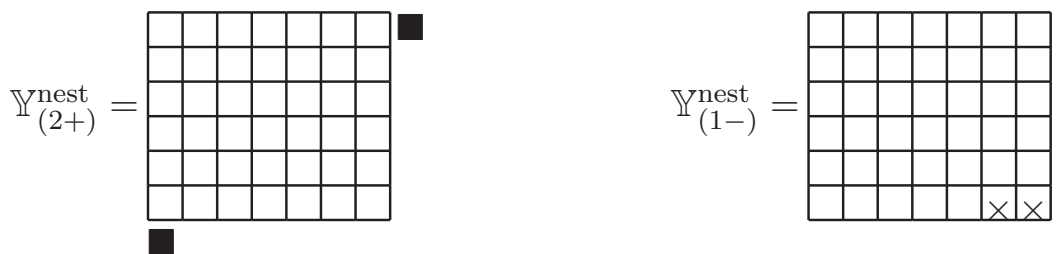
Here the two black cells form the only pair that can be added, while the two crossed cells form the only pair that can be removed.

Generally, in the language of nested-block hook diagrams (2.16)

$$
\mathbb{Y}^{\text {nest }}\{\underbrace{\mathbf{a}_{1}, \mathbf{a}_{1}-1, \ldots, \mathbf{a}_{1}-n_{1}+1}_{n_{1}}, \ldots, \underbrace{\mathbf{a}_{\mathbf{p}}, \mathbf{a}_{\mathbf{p}}-1, \ldots, \mathbf{a}_{\mathbf{p}}-n_{\mathbf{p}}+1}_{n_{\mathbf{p}}}\},
$$

one can either add two cells to a $\left(n_{1}+\ldots+n_{k-1}+1\right)$-th hook

$$
\mathbb{Y}_{k+}^{\text {nest }}\{\underbrace{\mathbf{a}_{1}, \mathbf{a}_{1}-1, \ldots, \mathbf{a}_{1}-n_{1}+1}_{n_{1}}, \ldots, \underbrace{\mathbf{a}_{k}+1}_{1}, \underbrace{\mathbf{a}_{k}-1, \ldots, \mathbf{a}_{k}-n_{k}+1}_{n_{k}-1}, \ldots, \underbrace{\mathbf{a}_{\mathbf{p}}, \ldots, \mathbf{a}_{\mathbf{p}}-n_{\mathbf{p}}+1}_{n_{\mathbf{p}}}\},
$$

or remove two cells from any $\left(n_{1}+\ldots+n_{k}\right)$-th hook

$$
\mathbb{Y}_{k-}^{\text {nest }}\{\underbrace{\mathbf{a}_{1}, \mathbf{a}_{1}-1, \ldots, \mathbf{a}_{1}-n_{1}+1}_{n_{1}}, \ldots, \underbrace{\mathbf{a}_{k}, \ldots, \mathbf{a}_{k}-n_{k}+2}_{n_{k}-1}, \underbrace{\mathbf{a}_{k}-n_{k}}_{1}, \ldots, \underbrace{\mathbf{a}_{\mathbf{p}}, \ldots, \mathbf{a}_{\mathbf{p}}-n_{\mathbf{p}}+1}_{n_{\mathbf{p}}}\}
$$

for any $k=1, \ldots, \mathbf{p}$ where $\mathbf{p}$ is the number of block hooks. If the $\mathbf{p}$-th block hook is not rectangular, i.e., $\mathbf{a}_{\mathbf{p}} \neq n_{\mathbf{p}}$, then two cells can also be added to the $\left(n_{1}+\ldots+n_{\mathbf{p}}+1\right)$-th zero hook as in (A.10)

$$
\mathbb{Y}_{(p+1)+}^{\text {nest }}\{\underbrace{\mathbf{a}_{1}, \ldots, \mathbf{a}_{1}-n_{1}+1}_{n_{1}}, \ldots, \underbrace{\mathbf{a}_{\mathbf{p}}, \ldots, \mathbf{a}_{\mathbf{p}}-n_{\mathbf{p}}+1}_{n_{\mathbf{p}}}, \underbrace{1}_{1}\} .
$$

Substitution of $\tilde{\mathbf{Y}}_{A}=\mathbb{Y}_{k+}^{\text {nest }}$ (A.12) into eq. (A.9) for $k \leq \mathbf{p}$ yields

$$
\mathcal{H}_{N_{k-1}+1}+\mathcal{H}_{\mathbf{a}_{k}+N_{k-1}+2} \leq \mathbf{r}
$$

where

$$
N_{s}=n_{1}+\ldots+n_{s}, \quad N_{0}=0 .
$$

A useful consequence of these inequalities is

$$
\mathcal{H}_{\mathbf{n}+1}+\mathcal{H}_{\mathbf{n}+2} \leq \mathbf{r}, \quad \mathbf{n}=\sharp\left(\mathbf{Y}_{A}\right)=N_{\mathbf{p}} .
$$

(Recall, that $\sharp\left(\mathbf{Y}_{A}\right)$ is the number of the nested hooks in $\mathbf{Y}_{A}$, while $\mathbf{p}$ is the number of the nested block hooks.) Indeed, for a non-rectangular last block hook, i.e., at $\mathbf{a}_{\mathbf{p}} \neq n_{\mathbf{p}}$, substitution of $\widetilde{\mathbf{Y}}_{A}=\mathbb{Y}_{(\mathbf{p}+1+)}^{\text {nest }}$ (A.14) into eq. (A.9) yields (A.17). At $\mathbf{a}_{\mathbf{p}}=n_{\mathbf{p}}$ (A.17) follows from (A.15) since in this case $\mathbf{a}_{\mathbf{p}}+N_{\mathbf{p}-1}=N_{\mathbf{p}}=\sharp\left(\mathbf{Y}_{A}\right)$ and $\mathcal{H}_{N_{\mathbf{p}}+1}=\mathcal{H}_{N_{\mathbf{p}-1}+1}$. Analogously, for $\widetilde{\mathbf{Y}}_{A}=\mathbb{Y}_{(k-)}^{\text {nest }}($ A.13), eq. (A.9) yields

$$
\mathcal{H}_{\mathbf{a}_{k}+N_{k-1}+1}+\mathcal{H}_{N_{k}} \geq \mathbf{r} \quad \forall k \leq \mathbf{p} .
$$

In particular, using that $N_{\mathbf{p}}=\sharp\left(\mathbf{Y}_{A}\right)$ and $\mathbf{a}_{k}+N_{k-1} \geq N_{\mathbf{p}}$, (A.18) yields

$$
\mathcal{H}_{\mathbf{n}}+\mathcal{H}_{\mathbf{n}+1} \geq \mathbf{r}, \quad \mathbf{n}=\sharp\left(\mathbf{Y}_{A}\right) .
$$


Now let us show that the addition of cells to $\mathbf{Y}^{\prime \prime}$ on the left from the $(\mathbf{n}+1)$-th column decreases $\chi^{\frac{1}{2}(\mathbf{r}-1)}$. Let

$$
\mathbf{Y}_{n_{1}, n_{2}, \ldots n_{k}+}:=\mathbf{Y} \cup \mathcal{S}\left(\mathrm{h}_{n_{1}}(\mathbf{Y})+1, n_{1}\right) \cup \ldots \cup \mathcal{S}\left(\mathrm{h}_{n_{k}}(\mathbf{Y})+1, n_{k}\right)
$$

for different $n_{i}$. We add here $k$ cells into $n_{1}, n_{2}, \ldots n_{k}$-th columns of $\mathbf{Y}$ at the condition that the resulting diagram exists.

Let

$$
\mathbf{Q}_{j_{1}, \ldots, j_{k}+}:=\chi^{\frac{1}{2}(\mathbf{r}-1)}\left(\left(\mathbf{Y}^{\prime \prime}{ }_{j_{1}, \ldots, j_{k}+}\right) \underset{\mathrm{SW}}{\otimes} \mathbf{Y}_{A}\right)-\chi^{\frac{1}{2}(\mathbf{r}-1)}\left(\mathbf{Y}^{\prime \prime} \underset{\mathrm{SW}}{\otimes} \mathbf{Y}_{A}\right)
$$

Properties of $\chi^{a}$ (2.3) and eq. (A.1) imply that $\mathbf{Q}_{j_{1}, \ldots, j_{k}+}=\sum_{i=1}^{k} \mathbf{Q}_{j_{i}+}$ and

$$
\mathbf{Q}_{j+}=\left(j-1-\mathfrak{h}_{j}\right)+\frac{1}{2}\left(\mathbf{r}-2 \mathcal{H}_{j}\right)-\frac{1}{2}
$$

where $\mathfrak{h}_{j}=\mathrm{h}_{j}\left(\mathbf{Y}_{A}\right)$. One can see that $\left(j-1-\mathfrak{h}_{j}\right) \leq 0$ for $j \leq \mathbf{n}+1$ because $\mathfrak{h}_{j} \geq \mathfrak{h}_{\mathbf{n}+1}$ while $\mathfrak{h}_{\mathbf{n}+1}=\mathbf{n}(\operatorname{cf}(2.13))$. Hence, taking into account eq. (A.19),

$$
\mathbf{Q}_{i, j+}=\chi^{\frac{1}{2}(\mathbf{r}-1)}\left(\mathbf{Y}^{\prime \prime}{ }_{i, j}+\underset{\mathrm{SW}}{\otimes} \mathbf{Y}_{A}\right)-\chi^{\frac{1}{2}(\mathbf{r}-1)}\left(\mathbf{Y}^{\prime \prime} \underset{\mathrm{SW}}{\otimes} \mathbf{Y}_{A}\right)<0 \quad \forall j<i \leq \mathbf{n}+1 .
$$

The following statements that hold by virtue of Lemma 1 and Young properties of tensor products will be used below. Any nonzero component of the tensor product $\mathbf{Y} \in$ $\mathbf{Y}_{0} \otimes \mathbf{Y}_{\delta}$ has to satisfy

$$
\begin{aligned}
& \mathrm{h}_{2 k}(\mathbf{Y})+\mathrm{h}_{2 k-1}(\mathbf{Y}) \leq \mathbf{r}+\mathrm{h}_{2 k}\left(\mathbf{Y}_{\delta}\right) \forall k \\
& \max \left(\mathrm{h}_{i}\left(\mathbf{Y}_{0}\right), \mathrm{h}_{i}\left(\mathbf{Y}_{\delta}\right)\right) \leq \mathrm{h}_{i}(\mathbf{Y}) \quad \forall i
\end{aligned}
$$

Eqs. (A.2), (A.17), (A.19) and (A.23) yield the following

Lemma 2 Given $\mathbf{Y}_{0}$ and $\mathbf{Y}_{A} \neq \bullet$ any solution $\mathbf{Y}^{\prime}$ of the homotopy equation

$$
\mathbf{Y}^{\prime}=\mathbf{Y}_{\mathrm{SW}}^{\prime \prime} \underset{\mathrm{Y}}{\mathrm{Y}}, \quad \mathbf{Y}^{\prime \prime} \in \mathbf{Y}_{0} \otimes \mathbf{Y}_{\delta}
$$

obeys the conditions

$$
\mathcal{H}_{2 k}+\mathcal{H}_{2 k-1}=\mathbf{r}+d_{k} \quad \forall k \leq \mathbf{m}:=\left[\frac{1}{2}(\mathbf{n}+1)\right], \quad \mathbf{n}=\sharp\left(\mathbf{Y}_{A}\right),
$$

where $d_{k}=\mathrm{h}_{2 k}\left(\mathbf{Y}_{\delta}\right), \mathcal{H}_{n}=\mathrm{h}_{n}\left(\mathbf{Y}^{\prime \prime}\right)$ and $[x]$ is the integer part of $x,[x] \leq x$.

We prove Lemma 2 by induction. To this end we observe that if inequalities (A.27) hold for $\forall k \leq \mathbf{j}$ with some $\mathbf{j} \leq \mathbf{m}$ then from the property that heights of columns of a YD do not increase from the left to the right it follows that if $d_{l}=d_{i}$ for some $l<i \leq \mathbf{j}$ then

$$
\mathcal{H}_{2 l}=\ldots=\mathcal{H}_{2 i-1}=\mathcal{H}_{2 i}
$$

and hence, if $\mathcal{H}_{2 l-1}>\mathcal{H}_{2 l}$ for some $l \leq \mathbf{j}$, then

$$
d_{l-1}>d_{l} \text { if } l \leq \mathbf{j} \text { and } d_{l}>d_{l+1} \text { if } l<\mathbf{j}
$$


and, if $\mathcal{H}_{2 l-2}>\mathcal{H}_{2 l-1}$ for some $l \leq \mathbf{j}$, then

$$
d_{l-1}>d_{l} .
$$

Here and after we use the convention $d_{0}:=\mathbf{r}$.

Firstly let us prove (A.27) at $k=1$. Suppose that this is not true, i.e.,

$$
\mathcal{H}_{1}+\mathcal{H}_{2}<\mathbf{r}+d_{1} .
$$

From (A.25), (A.31) it follows that $d_{1}<\mathbf{r}$. This demands $\mathcal{H}_{1}<\mathbf{r}$ since otherwise $\mathcal{H}_{2}<$ $d_{1}<\mathbf{r}$ in contradiction with (A.25). Therefore $\mathbf{r} \geq \mathcal{H}_{1}+1 \geq \mathcal{H}_{2}+1$ and $\mathbf{r} \geq d_{1}+1$. Hence YD $\mathbf{Y}_{1,2+}^{\prime \prime}$ and $\mathbf{Y}_{\delta 1,2+}$ (A.20) are nonzero. Since by virtue of (A.31)

$$
\mathcal{H}_{1}+\mathcal{H}_{2}+2 \leq \mathbf{r}+\left(d_{1}+1\right)
$$

i.e., conditions (A.24) hold, $\mathbf{Y}_{1,2+}^{\prime \prime}$ is a nonzero component of $\mathbf{Y}_{0} \otimes \mathbf{Y}_{\delta 1,2+}$. Substitution of $\mathbf{Y}^{\prime \prime}{ }_{1,2+}$ into (A.23) yields by virtue of (A.9) that $\chi^{\frac{1}{2}(\mathbf{r}-1)}\left(\mathbf{Y}^{\prime \prime} \underset{\mathrm{SW}}{\otimes} \mathbf{Y}_{A}\right)>0$ in contradiction with the assumption of Lemma 2. This proves (A.27) at $k=1$.

Now suppose that (A.27) holds for all $k \leq \mathbf{j}$ with some $\mathbf{j} \geq 1$ but does not hold for $k=\mathbf{j}+1$, i.e., taking into account Lemma 1 ,

$$
\begin{aligned}
\mathcal{H}_{2 \mathbf{j}+1}+\mathcal{H}_{2 \mathbf{j}+2} & <\mathbf{r}+d_{\mathbf{j}+1}, \\
\mathcal{H}_{2 k+1}+\mathcal{H}_{2 k+2} & =\mathbf{r}+d_{k+1} \quad \forall k<\mathbf{j} .
\end{aligned}
$$

If $\mathcal{H}_{2 \mathbf{j}}=\mathbf{r}$ then as for the case of $k=1$ eqs. (A.25), (A.32) yield $d_{\mathbf{j}}=\mathbf{r}, \mathcal{H}_{2 \mathbf{j}+1}<\mathbf{r}$ and $d_{\mathbf{j}+1}<\mathbf{r}$. Therefore, substitution of $\mathbf{Y}^{\prime \prime}{ }_{(2 \mathbf{j}+1),(2 \mathbf{j}+2)+}$ into (A.23) leads by virtue of (A.9) to the contradiction with the assumption of Lemma 2. Hence it remains to consider the case of $\mathcal{H}_{2 \mathbf{j}}<\mathbf{r}, d_{\mathbf{j}}<\mathbf{r}$.

By eq. (A.28), assumption (A.32) implies that at least one of the equalities $\mathcal{H}_{2 \mathbf{j}-1}=$ $\mathcal{H}_{2 \mathbf{j}}=\mathcal{H}_{2 \mathbf{j}+1}=\mathcal{H}_{2 \mathbf{j}+2}$ fails. Consider different cases.

Let $\mathcal{H}_{2 \mathbf{j}}>\mathcal{H}_{2 \mathbf{j}+1}$. If $d_{\mathbf{j}}>d_{\mathbf{j}+1}$ then the YD $\mathbf{Y}^{\prime \prime}{ }_{2 \mathbf{j}+1,2 \mathbf{j}+2+}$ and $\mathbf{Y}_{\delta 2 \mathbf{j}+1,2 \mathbf{j}+2+}$ have an allowed shape and are nonzero since conditions (A.24) hold for them by virtue of (A.32). Hence $\mathbf{Y}^{\prime \prime}{ }_{2 \mathbf{j}+1,2 \mathbf{j}+2+} \in \mathbf{Y}_{0} \otimes \mathbf{Y}_{\delta 2 \mathbf{j}+1,2 \mathbf{j}+2+}$ and (A.23) yields $\chi^{\frac{1}{2}(\mathbf{r}-1)}\left(\mathbf{Y}^{\prime \prime} \underset{\mathrm{SW}}{\otimes} \mathbf{Y}_{A}\right)>0$ in contradiction with the assumption of Lemma 2.

If $d_{\mathbf{j}}=d_{\mathbf{j}+1}$ then by virtue of (A.29), (A.30) there exist some $n \leq \mathbf{j}$ and $m \in[2 n-1,2 n]$ such that $\mathbf{Y}^{\prime \prime}{ }_{m, 2 \mathbf{j}+1}+$ and $\mathbf{Y}_{\delta 2 n-1,2 n}+$ are nonzero $\mathrm{YD}$ satisfying (A.24) by virtue of (A.32) and (A.33). Hence $\mathbf{Y}^{\prime \prime}{ }_{m, 2 \mathbf{j}+1+} \in \mathbf{Y}_{0} \otimes \mathbf{Y}_{\delta 2 n-1,2 n+}$ in contradiction with the assumption of Lemma 2.

This allows us to set $\mathcal{H}_{2 \mathbf{j}}=\mathcal{H}_{2 \mathbf{j}+1}$.

If $\mathcal{H}_{2 \mathbf{j}-1}>\mathcal{H}_{2 \mathbf{j}}$, (A.29) implies $d_{\mathbf{j}-1}>d_{\mathbf{j}}$ and there exist nonzero YD $\mathbf{Y}^{\prime \prime}{ }_{2 \mathbf{j}, 2 \mathbf{j}+1+}$ and $\mathbf{Y}_{\delta 2 \mathbf{j}-1,2 \mathbf{j}+}$ satisfying (A.24) by virtue of (A.32) and (A.33). Then $\mathbf{Y}^{\prime \prime}{ }_{2 \mathbf{j}, 2 \mathbf{j}+1+} \in \mathbf{Y}_{0} \otimes$ $\mathbf{Y}_{\delta 2 \mathbf{j}-1,2 \mathbf{j}}+$ which by (A.23) is in contradiction with the assumption of Lemma 2.

If $\mathcal{H}_{2 \mathbf{j}-1}=\mathcal{H}_{2 \mathbf{j}}$, then the only remaining option is $\mathcal{H}_{2 \mathbf{j}+1}>\mathcal{H}_{2 \mathbf{j}+2}$. Hence by (A.29), (A.30) there exist some $n<\mathbf{j}$ and $m \in[2 n-1,2 n]$ such that $\mathbf{Y}^{\prime \prime}{ }_{m, 2 \mathbf{j}+2+}$ and $\mathbf{Y}_{\delta 2 n-1,2 n+}$ are nonzero YD satisfying (A.24) by virtue of (A.32) and (A.33). Then 
$\mathbf{Y}_{m, 2 \mathbf{j}+2+}^{\prime \prime} \in \mathbf{Y}_{0} \otimes \mathbf{Y}_{\delta 2 n-1,2 n+}$ in contradiction with the assumption of Lemma 2 thus completing the proof.

It is convenient to introduce notations

$$
\mathbb{H}_{j}=\mathbf{r}-\mathcal{H}_{j} \quad \forall j \leq 2 \mathbf{m} .
$$

Substitution of $\mathbb{H}_{j}$ (A.34) into (A.15), (A.17) and (A.18) yields inequalities on $\mathbb{H}_{j}$ with $j \leq 2 \mathbf{m}$ and $\mathcal{H}_{k}$ with $k>2 \mathbf{m}$. In particular, (A.15) and (A.18) yield, respectively,

$$
\begin{aligned}
\mathbb{H}_{N_{k-1}+1} & \geq \mathcal{H}_{\mathbf{a}_{k}+N_{k-1}+2}, \quad k \leq \mathbf{p}, \\
\mathbb{H}_{N_{k}} & \leq \mathcal{H}_{\mathbf{a}_{k}+N_{k-1}+1}, \quad k \leq \mathbf{p} .
\end{aligned}
$$

Since by construction

$$
\mathbb{H}_{k+1} \geq \mathbb{H}_{k} \quad \forall k<2 \mathbf{m}, \quad \mathcal{H}_{j} \geq \mathcal{H}_{j+1} \quad \forall j \geq 2 \mathbf{m}+1
$$

eqs. (A.35)-(A.37) give the following chain of inequalities

$$
\begin{aligned}
\ldots & \geq \mathcal{H}_{\mathbf{a}_{k}+N_{k-1}+1} \geq \mathbb{H}_{N_{k}} \geq \ldots \geq \mathbb{H}_{N_{k-1}+1} \geq \mathcal{H}_{\mathbf{a}_{k}+N_{k-1}+2} \geq \ldots \\
& \geq \mathcal{H}_{\mathbf{a}_{k+1}+N_{k}+1} \geq \mathbb{H}_{N_{k+1}} \geq \ldots,
\end{aligned}
$$

where the starting point depends on the oddness of $\mathbf{n} \equiv N_{\mathbf{p}}$ and on whether $a_{\mathbf{p}}-n_{\mathbf{p}}$ is zero or not. There are two reasons for this. Firstly there is a difference between diagrams with rectangular $\left(a_{\mathbf{p}}=n_{\mathbf{p}}\right)$ and not rectangular $\left(a_{\mathbf{p}}>n_{\mathbf{p}}\right)$ inner block hooks that gives different numbers of inequalities on $\mathcal{H}$ in these two cases. Secondly, $2 \mathbf{m}=N_{\mathbf{p}}+1$ for odd $\mathbf{n}$ and $2 \mathbf{m}=N_{\mathbf{p}}$ for even $\mathbf{n}$, that leads to different relations between $\mathbb{H}_{j}$ and $\mathcal{H}_{2 \mathbf{m}+i}$.

Namely for even $\mathbf{n}$ and $a_{\mathbf{p}}>n_{\mathbf{p}}$

$$
\mathcal{H}_{N_{p}+1} \geq \ldots \geq \mathcal{H}_{\mathbf{a}_{p}+N_{p-1}+1} \geq \mathbb{H}_{N_{p}} \geq \ldots \geq \mathbb{H}_{N_{p-1}+1} \geq \mathcal{H}_{\mathbf{a}_{p}+N_{p-1}+2} \geq \ldots
$$

For odd $\mathbf{n}$ and $a_{\mathbf{p}}>n_{\mathbf{p}}$

$\mathbb{H}_{N_{p}+1} \geq \mathcal{H}_{N_{p}+2} \geq \ldots \geq \mathcal{H}_{\mathbf{a}_{p}+N_{p-1}+1} \geq \mathbb{H}_{N_{p}} \geq \ldots \geq \mathbb{H}_{N_{p-1}+1} \geq \mathcal{H}_{\mathbf{a}_{p}+N_{p-1}+2} \geq \ldots$

For even $\mathbf{n}$ and $a_{\mathbf{p}}=n_{\mathbf{p}}$

$$
\mathcal{H}_{N_{p}+1} \geq \mathbb{H}_{N_{p}} \geq \ldots \geq \mathbb{H}_{N_{p-1}+1} \geq \mathcal{H}_{\mathbf{a}_{p}+N_{p-1}+2} \geq \ldots
$$

For odd $\mathbf{n}$ and $a_{\mathbf{p}}=n_{\mathbf{p}}$

$$
\mathbb{H}_{N_{p}+1} \geq \ldots \geq \mathbb{H}_{N_{p-1}+1} \geq \mathcal{H}_{N_{p}+2} \geq \ldots \geq \mathcal{H}_{\mathbf{a}_{p}+N_{p-1}+1} \geq \mathbb{H}_{N_{p-1}} \geq \ldots
$$

To prove Theorem 1 (see pg. 17) we first prove

Lemma 3 Given $\mathbf{Y}_{0}$ and $\mathbf{Y}_{A} \neq \bullet$, any solution $\mathbf{Y}^{\prime}$ of the homotopy equation

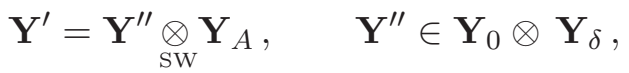

obeys relations (3.50) of Theorem 1. 
Firstly let us assume that

$$
\mathrm{I}_{1}\left(Y_{\delta}\right)=2 \mathbf{m} .
$$

(Recall that $\mathbf{m}=\left[\frac{1}{2}(\mathbf{n}+1)\right], \mathbf{n}=\sharp\left(\mathbf{Y}_{A}\right)(3.51)$.) Since $\mathbf{Y}^{\prime \prime}\left[\mathcal{H}_{1}, \ldots\right] \in \mathbf{Y}_{0} \otimes Y_{\delta}$,

$$
\sum_{i} \mathcal{H}_{i}=\sum_{j} \mathrm{~h}_{j}\left(\mathbf{Y}_{0}\right)+\sum_{k} \mathrm{~h}_{k}\left(\mathbf{Y}_{\delta}\right)
$$

where summation is over all columns of each diagram. Since by virtue of eqs. (A.27) and (A.34)

$$
\mathcal{H}_{2 i-1}=d_{i}+\mathbb{H}_{2 i}, \quad \mathcal{H}_{2 i}=d_{i}+\mathbb{H}_{2 i-1}, \quad i \leq \mathbf{m},
$$

the substitution of (A.46) into (A.45) yields using (A.44)

$$
\sum_{i=1}^{2 \mathrm{~m}} \mathbb{H}_{i}+\sum_{i>2 \mathbf{m}} \mathcal{H}_{i}=\sum_{j} h_{j}, \quad h_{j}=\mathrm{h}_{j}\left(\mathbf{Y}_{0}\right)
$$

Let

$$
T^{B_{1}\left[h_{1}\right], B_{2}\left[h_{2}\right] \ldots}, \quad D^{C_{1}\left[d_{1}\right], \ldots, C_{2 \mathbf{m}}\left[d_{\mathbf{m}}\right]}
$$

be tensors with symmetry properties of given traceless $\mathrm{YD}_{0}\left[h_{1}, h_{2}, \ldots\right]$ and Kronecker YD $\mathbf{Y}_{\delta}\left[d_{1}, d_{1}, d_{2}, d_{2}, \ldots\right]$ (3.51), respectively, while

$$
F_{A_{1}\left[\mathcal{H}_{1}\right], A_{2}\left[\mathcal{H}_{2}\right], \ldots}
$$

be a tensor with symmetry of $\mathbf{Y}^{\prime \prime}\left[\mathcal{H}_{1}, \mathcal{H}_{2}, \ldots\right]=\mathbf{Y}^{\prime \prime}\left\{\mathbf{Y}_{0}, \mathbf{Y}_{\delta}\right\} \in \mathbf{Y}_{0} \otimes \mathbf{Y}_{\delta}$. From (A.44) and Lemma 2 it follows that $\mathbf{Y}_{\delta}$ in (A.3) is determined by (A.27). Since $\mathbf{Y}^{\prime \prime}$ is a component of the tensor product $\mathbf{Y}_{0} \otimes \mathbf{Y}_{\delta}$, one can contract all indices of $D$ (A.48) with indices of $F$ (A.49) obtaining by virtue of (A.46) the tensor

$$
\mathcal{F}_{A_{1}\left[\mathbb{H}_{2}\right] ; A_{2}\left[\mathbb{H}_{1}\right] ; \ldots ; A_{2 \mathbf{m}-1}\left[\mathbb{H}_{2 \mathbf{m}}\right] ; A_{2 \mathbf{m}}\left[\mathbb{H}_{2 \mathbf{m}-1}\right] ; A_{2 \mathbf{m}+1}\left[\mathcal{H}_{2 \mathbf{m}+1}\right], A_{2 \mathbf{m}+2}\left[\mathcal{H}_{2 \mathbf{m}+2}\right], \ldots},
$$

which is antisymmetric in all indices $A_{j}[$.$] but not necessarily has properties of a YD.$ The numbers of antisymmetrized indices $A_{j}[$.$] are called 'heights' of \mathcal{F}$. In accordance with (A.47) all indices of $\mathcal{F}$ (A.50) have to be contracted with all indices of $T$ (A.48). Hence the maximal height of $\mathcal{F}$ cannot be greater than $h_{1}=\mathrm{h}_{1}\left(\mathbf{Y}_{0}\right)$. On the other hand, the SW-principle demands the maximal height of $\mathcal{F}$ be exactly equal to $h_{1}$. Analogously, the subleading height cannot be greater than $h_{2}=\mathrm{h}_{2}\left(\mathbf{Y}_{0}\right)$, etc. Hence the SW-principle demands ordered heights of $\mathcal{F}$ be equal to $h_{i}$. Since the ordering of heights of $\mathcal{F}$ is determined by inequalities (A.38)-(A.42) this expresses the heights of $\mathcal{F}$ via $h_{i}$. Straightforward computation gives $\mathcal{H}_{j}$ in the form (3.50). This completes the proof at the condition that (A.44) holds.

To show that condition (A.44) holds true it is enough to show that existence of such a solution $\mathbf{Y}^{\prime}\left[\mathcal{H}_{1}, \mathcal{H}_{2}, \ldots\right]$ of eq. (5.23) that

$$
\begin{aligned}
& \mathbf{Y}^{\prime}=\mathbf{Y}_{\mathrm{SW}}^{\prime \prime} \otimes \mathbf{Y}_{A}, \quad \mathbf{Y}^{\prime \prime} \in \mathbf{Y}_{0} \otimes \mathbf{Y}_{\delta}, \quad \mathbf{Y}_{\delta}=\mathbf{Y}_{\delta}\left[d_{1}, d_{1}, \ldots, d_{K}, d_{K}\right], \\
& d_{k}=\mathcal{H}_{2 k}+\mathcal{H}_{2 k-1}-\mathbf{r} \quad \forall k \leq \mathbf{m}, \quad K \geq \mathbf{m}+1, \quad d_{K}>0, \quad \mathcal{H}_{j}=\mathrm{h}_{j}\left(\mathbf{Y}^{\prime \prime}\right)
\end{aligned}
$$

contradicts the assumption that $\mathbf{Y}^{\prime}$ solves eq. (5.23). 
Since

$$
\mathbf{Y}_{\delta} \in \mathbf{Y}_{\delta}^{-} \otimes \mathbf{Y}_{\delta}[1,1], \quad \mathbf{Y}_{\delta}^{-}:=\mathbf{Y}_{\delta}^{-}\left[d_{1}, d_{1}, \ldots, d_{K}-1, d_{K}-1\right]
$$

then $\mathbf{Y}^{\prime \prime} \in \mathbf{Y}_{0} \otimes \mathbf{Y}_{\delta} \in \mathbf{Y}_{0} \otimes \mathbf{Y}_{\delta}^{-} \otimes \mathbf{Y}_{\delta}[1,1]$ and by associativity of the tensor product

$$
\mathbf{Y}^{\prime \prime} \in \mathbf{Y}^{\prime \prime-} \otimes \mathbf{Y}_{\delta}[1,1], \quad \mathbf{Y}^{\prime \prime-} \in \mathbf{Y}_{0} \otimes \mathbf{Y}_{\delta}^{-} .
$$

By construction, $\mathbf{Y}^{\prime \prime}=\mathbf{Y}^{\prime \prime}-\cup \mathcal{S}\left(\mathcal{H}_{j_{1}}, j_{1}\right) \cup \mathcal{S}\left(\mathcal{H}_{j_{2}}, j_{2}\right)$ for some $j_{1} \neq j_{2}$ since $\mathbf{Y}_{\delta}[1,1]$ is symmetric. For definiteness we set $j_{1}<j_{2}$. Additivity of $\chi^{a}$ (2.3) and eq. (A.1) imply that

$$
\chi^{\frac{1}{2}(\mathbf{r}-1)}\left(\mathbf{Y}^{\prime \prime} \underset{\mathrm{SW}}{\otimes} \mathbf{Y}_{A}\right)-\chi^{\frac{1}{2}(\mathbf{r}-1)}\left(\mathbf{Y}^{\prime \prime-} \underset{\mathrm{SW}}{\otimes} \mathbf{Y}_{A}\right)=j_{1}-1-\mathfrak{h}_{j_{1}}+j_{2}-\mathfrak{h}_{j_{2}}+\mathbf{r}-\mathcal{H}_{j_{1}}-\mathcal{H}_{j_{2}}
$$

where $\mathfrak{h}_{j}=\mathrm{h}_{j}\left(\mathbf{Y}_{A}\right)$. Consider different cases.

Let $j_{1} \geq \mathbf{n}+1$. Then, by properties of almost symmetric YD, $j_{1}-1-\mathfrak{h}_{j_{1}} \geq 0$ because $\mathfrak{h}_{j_{1}} \leq \mathfrak{h}_{\mathbf{n}+1}$ while $\mathfrak{h}_{\mathbf{n}+1}=\mathbf{n}$ (cf eq. (2.13)). Analogously, $j_{2}-\mathfrak{h}_{j_{2}}>0$ since $j_{2}>j_{1}$. By virtue of (A.17) $\mathbf{r}-\mathcal{H}_{j_{1}}-\mathcal{H}_{j_{2}} \geq 0$. Hence eq. (A.55) yields $\chi^{\frac{1}{2}(\mathbf{r}-1)}\left(\mathbf{Y}_{\mathrm{SW}}^{\prime \prime} \underset{\mathrm{SW}}{\otimes} \mathbf{Y}_{A}\right)>$ $\chi^{\frac{1}{2}(\mathbf{r}-1)}\left(\mathbf{Y}^{\prime \prime}-\underset{\mathrm{SW}}{\otimes} \mathbf{Y}_{A}\right)$. By (A.9) this contradicts the assumption that $\mathbf{Y}^{\prime}$ solves Homotopy condition.

Let $j_{1} \leq 2 \mathbf{m}$. Since $\mathbf{Y}^{\prime \prime}$ satisfies (A.27), this is equivalent to

$$
\begin{aligned}
\mathrm{h}_{2 l-1}\left(\mathbf{Y}^{\prime \prime-}\right)+\mathrm{h}_{2 l}\left(\mathbf{Y}^{\prime \prime-}\right) & =\mathbf{r}+d_{l}-1 & & \text { for } l=\left[\frac{1}{2}\left(j_{1}+1\right)\right], \\
\mathrm{h}_{2 k-1}\left(\mathbf{Y}^{\prime \prime-}\right)+\mathrm{h}_{2 k}\left(\mathbf{Y}^{\prime \prime-}\right) & =\mathbf{r}+d_{k} & & \text { for } k \leq \mathbf{m}, k \neq l .
\end{aligned}
$$

It can be shown along the lines of the proof of Lemma 2 that there exist YD $\mathbf{Y}^{\prime \prime-}{ }^{m, j_{1}+}$ with some $m \leq 2 l$ and some Kronecker $\mathrm{YD} \tilde{\mathbf{Y}}_{\delta} \supset \mathbf{Y}_{\delta}^{-}$such that $\mathbf{Y}^{\prime \prime-}{ }_{m, j_{1}+} \in \mathbf{Y}_{0} \otimes \widetilde{\mathbf{Y}}_{\delta}$. By construction $\mathbf{Y}^{\prime \prime-}{ }_{m, j_{1}}+\prec \mathbf{Y}^{\prime \prime}$ in contradiction with the assumption that $\mathbf{Y}^{\prime}$ (A.51) solves Homotopy condition. This completes the proof of Lemma 3.

To complete the proof of Theorem 1 (see pg. 17) it remains to prove

Lemma 4 Differential forms obeying symmetry properties of $Y D \mathbf{Y}^{\prime}$ (3.49) are $\sigma_{-}^{\mathbf{r}}$-closed but not $\sigma_{-}^{\mathbf{r}}$-exact.

Indeed, consider a tensor $P(\xi, Y)$ with symmetry properties of $\mathrm{YD} \mathbf{Y}^{\prime}=\left(\mathbf{Y}_{0} \otimes\right.$ $\left.\mathbf{Y}_{\delta}\right) \underset{\mathrm{SW}}{\otimes} \mathbf{Y}_{A}$ (3.49). The action of $\sigma_{-}^{\mathbf{r}}=\delta_{i j} \xi^{A B} \frac{\partial^{2}}{\partial Y_{i}^{A} \partial Y_{j}^{B}}$ (1.5) on $P(\xi, Y)$ (3.39) yields zero by virtue of one of the following three mechanisms

- antisymmetrization of the indices of $\xi^{A B}$ in $\sigma_{-}$with those of the almost symmetric YD $\mathbf{Y}_{A}$ gives zero allowing no proper contraction of the resulting almost symmetric YDs within the representation of $P(\xi, Y \mid X)(3.39)$.

- contraction of the indices of the Kronecker symbol with those of a traceless diagram,

- antisymmetrization of the lower case Latin indices of the Kronecker symbol with the indices of $\mathbf{Y}_{\delta}(3.51)$ gives zero by virtue of Lemma 1.

To show that ker $\Delta(5.1)$ does not contain $\sigma_{-}^{\mathbf{r}}$-exact elements, suppose that $\Delta a=0$, $a=\sigma_{-}^{\mathbf{r}} b$ for some $b$. Since $\left[\sigma_{-}^{\mathbf{r}}, \Delta\right]=0$, the expansion of $b$ in eigenvectors of $\Delta$ can only contain those with zero eigenvalues, i.e., $\Delta b=0$. Since it is shown that every $b$ obeying this condition is $\sigma_{-}^{\mathbf{r}}$-closed, every exact $a \in \operatorname{ker} \Delta$ is zero. 
Open Access. This article is distributed under the terms of the Creative Commons Attribution License (CC-BY 4.0), which permits any use, distribution and reproduction in any medium, provided the original author(s) and source are credited.

\section{References}

[1] C. Fronsdal, Massless Particles, Ortosymplectic Symmetry and Another Type of Kaluza-Klein Theory, Preprint UCLA/85/TEP/10, in Mathematical Physics Studies. Vol. 8: Essays on Supersymmetry, Reidel Publishing, Dordrecht Netherlands (1986).

[2] I.A. Bandos and J. Lukierski, Tensorial central charges and new superparticle models with fundamental spinor coordinates, Mod. Phys. Lett. A 14 (1999) 1257 [hep-th/9811022] [INSPIRE].

[3] I.A. Bandos, J. Lukierski and D.P. Sorokin, Superparticle models with tensorial central charges, Phys. Rev. D 61 (2000) 045002 [hep-th/9904109] [INSPIRE].

[4] M.A. Vasiliev, Conformal higher spin symmetries of $4-d$ massless supermultiplets and osp (L,2M) invariant equations in generalized (super) space, Phys. Rev. D 66 (2002) 066006 [hep-th/0106149] [INSPIRE].

[5] O.A. Gelfond and M.A. Vasiliev, Higher rank conformal fields in the $\operatorname{Sp}(2 M)$ symmetric generalized space-time, Theor. Math. Phys. 145 (2005) 1400 [hep-th/0304020] [INSPIRE].

[6] M.A. Vasiliev, Higher spin conserved currents in $\mathrm{Sp}(2 M)$ symmetric space-time, Russ. Phys. J. 45 (2002) 670 [hep-th/0204167] [INSPIRE].

[7] M.A. Vasiliev, Relativity, causality, locality, quantization and duality in the $\operatorname{Sp}(2 M)$ invariant generalized space-time, hep-th/0111119 [INSPIRE].

[8] I. Bandos, X. Bekaert, J.A. de Azcarraga, D. Sorokin and M. Tsulaia, Dynamics of higher spin fields and tensorial space, JHEP 05 (2005) 031 [hep-th/0501113] [INSPIRE].

[9] M.A. Vasiliev, Consistent equations for interacting massless fields of all spins in the first order in curvatures, Annals Phys. 190 (1989) 59 [INSPIRE].

[10] O.A. Gelfond and M.A. Vasiliev, Operator algebra of free conformal currents via twistors, Nucl. Phys. B 876 (2013) 871 [arXiv:1301.3123] [INSPIRE].

[11] J.M. Maldacena, The large- $N$ limit of superconformal field theories and supergravity, Int. J. Theor. Phys. 38 (1999) 1113 [hep-th/9711200] [INSPIRE].

[12] S.S. Gubser, I.R. Klebanov and A.M. Polyakov, Gauge theory correlators from noncritical string theory, Phys. Lett. B 428 (1998) 105 [hep-th/9802109] [INSPIRE].

[13] E. Witten, Anti-de Sitter space and holography, Adv. Theor. Math. Phys. 2 (1998) 253 [hep-th/9802150] [INSPIRE].

[14] I.R. Klebanov and A.M. Polyakov, AdS dual of the critical $O(N)$ vector model, Phys. Lett. B 550 (2002) 213 [hep-th/0210114] [INSPIRE].

[15] E. Sezgin and P. Sundell, Holography in $4 D$ (super) higher spin theories and a test via cubic scalar couplings, JHEP 07 (2005) 044 [hep-th/0305040] [INSPIRE].

[16] S. Giombi and X. Yin, Higher spin gauge theory and holography: the three-point functions, JHEP 09 (2010) 115 [arXiv:0912.3462] [INSPIRE].

[17] S. Giombi and X. Yin, Higher spins in AdS and twistorial holography, JHEP 04 (2011) 086 [arXiv: 1004.3736] [INSPIRE]. 
[18] J. Maldacena and A. Zhiboedov, Constraining Conformal Field Theories with A Higher Spin Symmetry, J. Phys. A 46 (2013) 214011 [arXiv:1112.1016] [InSPIRE].

[19] J. Maldacena and A. Zhiboedov, Constraining conformal field theories with a slightly broken higher spin symmetry, Class. Quant. Grav. 30 (2013) 104003 [arXiv:1204.3882] [INSPIRE].

[20] S. Giombi and X. Yin, The Higher Spin/Vector Model Duality, J. Phys. A 46 (2013) 214003 [arXiv: 1208.4036] [INSPIRE].

[21] M.R. Gaberdiel and R. Gopakumar, Minimal Model Holography, J. Phys. A 46 (2013) 214002 [arXiv:1207.6697] [inSPIRE].

[22] M.A. Vasiliev, Holography, Unfolding and Higher-Spin Theory, J. Phys. A 46 (2013) 214013 [arXiv: 1203.5554] [INSPIRE].

[23] K. Alkalaev, Mixed-symmetry tensor conserved currents and AdS/CFT correspondence, J. Phys. A 46 (2013) 214007 [arXiv:1207.1079] [InSPIRE].

[24] V.E. Didenko and E.D. Skvortsov, Towards higher-spin holography in ambient space of any dimension, J. Phys. A 46 (2013) 214010 [arXiv:1207.6786] [INSPIRE].

[25] R.R. Metsaev, CFT adapted approach to massless fermionic fields, AdS/CFT and fermionic conformal fields, arXiv:1311.7350 [INSPIRE].

[26] O.A. Gelfond and M.A. Vasiliev, Unfolded Equations for Current Interactions of $4 d$ Massless Fields as a Free System in Mixed Dimensions, J. Exp. Theor. Phys. 120 (2015) 484 [arXiv: 1012.3143] [INSPIRE].

[27] H. Elvang and Y.-t. Huang, Scattering Amplitudes, arXiv:1308.1697 [INSPIRE].

[28] A.K. Aiston and H.R. Morton, Idempotents of Hecke algebras of type A, q-alg/9702017.

[29] O.V. Shaynkman and M.A. Vasiliev, Scalar field in any dimension from the higher spin gauge theory perspective, Theor. Math. Phys. 123 (2000) 683 [hep-th/0003123] [INSPIRE].

[30] X. Bekaert, S. Cnockaert, C. Iazeolla and M.A. Vasiliev, Nonlinear higher spin theories in various dimensions, hep-th/0503128 [INSPIRE].

[31] M. Günaydin and D. Minic, Singletons, doubletons and M-theory, Nucl. Phys. B 523 (1998) 145 [hep-th/9802047] [INSPIRE].

[32] M. Günaydin, Unitary supermultiplets of $\mathrm{OSp}(1 / 32, R)$ and $M$-theory, Nucl. Phys. B 528 (1998) 432 [hep-th/9803138] [INSPIRE].

[33] R. Howe, Remarks on classical invariant theory, Trans. Amer. Math. Soc. 313 (1989) 539.

[34] M.A. Vasiliev, Bosonic conformal higher-spin fields of any symmetry, Nucl. Phys. B 829 (2010) 176 [arXiv:0909.5226] [InSPIRE].

[35] O.A. Gelfond and M.A. Vasiliev, Higher Spin Fields in Siegel Space, Currents and Theta Functions, JHEP 03 (2009) 125 [arXiv:0801.2191] [INSPIRE].

[36] M.A. Vasiliev, Multiparticle extension of the higher-spin algebra, Class. Quant. Grav. 30 (2013) 104006 [arXiv:1212.6071] [INSPIRE].

[37] N. Arkani-Hamed, J.L. Bourjaily, F. Cachazo, A. Postnikov and J. Trnka, On-Shell Structures of MHV Amplitudes Beyond the Planar Limit, JHEP 06 (2015) 179 [arXiv: 1412.8475] [INSPIRE].

[38] L.V. Bork and A.I. Onishchenko, Wilson lines, Grassmannians and Gauge Invariant Off-shell Amplitudes in $N=4$ SYM, arXiv:1607.02320 [INSPIRE]. 